\title{
Domain Engineering for Graph
}

\section{Libraries}

By

Bo Tian

\author{
A thesis submitted to \\ the Faculty of Graduate Studies and Research \\ in partial fulfilment of \\ the requirements for the degree of \\ Master of Computer Science
}

Ottawa-Carleton Institute for Computer Science

School of Computer Science

Carleton University

Ottawa, Ontario

January 2004

(C) Copyright 2004, Bo Tian 


\author{
National Library \\ of Canada \\ Acquisitions and \\ Bibliographic Services \\ 395 Wellington Street \\ Ottawa ON K1A ON4 \\ Canada
}

Bibliothèque nationale

du Canada

Acquisisitons et services bibliographiques

395 , rue Wellington Ottawa ON K1A ON4 Canada
Your file Votre référence ISBN: 0-612-89866-0

Ourfile Notre référence ISBN: 0-612-89866-0
The author has granted a nonexclusive licence allowing the National Library of Canada to reproduce, loan, distribute or sell copies of this thesis in microform, paper or electronic formats.

The author retains ownership of the copyright in this thesis. Neither the thesis nor substantial extracts from it may be printed or otherwise reproduced without the author's permission.
L'auteur a accordé une licence non exclusive permettant à la Bibliothèque nationale du Canada de reproduire, prêter, distribuer ou vendre des copies de cette thèse sous la forme de microfiche/film, de reproduction sur papier ou sur format électronique.

L'auteur conserve la propriété du droit d'auteur qui protège cette thèse. $\mathrm{Ni}$ la thèse ni des extraits substantiels de celle-ci ne doivent être imprimés ou aturement reproduits sans son autorisation.
In compliance with the Canadian Privacy Act some supporting forms may have been removed from this dissertation.

While these forms may be included in the document page count, their removal does not represent any loss of content from the dissertation.
Conformément à la loi canadienne sur la protection de la vie privée, quelques formulaires secondaires ont été enlevés de ce manuscrit.

Bien que ces formulaires aient inclus dans la pagination, il n'y aura aucun contenu manquant. 


\section{Acknowledgements}

First I would like to thank my advisor, Jean-Pierre Corriveau. He gave me support throughout these years in many ways. I just cannot find a way to thank him.

I am also thankful to my fellow graduate students. They gave suggestions to this dissertation and helped me to improve my presentation.

I couldn't finish my research without the support from my wife. She is always behind me, silently and firmly.

Finally I would like to dedicate this dissertation to my father. In his time, going to university was a dream. I hope he can share my happiness. 


\begin{abstract}
In this thesis, the problem we tackle is how to facilitate the reuse of a set of independently developed $\mathrm{C}++$ graph libraries. We are concerned more precisely with library users being offered an alternative to having to study in detail the code of a library before deciding whether or not and how to reuse its components. Our fundamental postulate is that domain engineering does facilitate reuse, as extensively argued in the corresponding literature. Thus, the question we ask is the following: can the notions of domain engineering, and more specifically the technique of feature modeling, be applied to a set of independently developed $\mathrm{C}++$ graph libraries (in order to facilitate reusing these libraries)? Our thesis is that they can and we demonstrate this by building feature models addressing the representational aspects of a family of independently developed graph libraries.
\end{abstract}

First, we develop a partial feature diagram for each graph library. Our intention here is to have these models provide a conceptual "road map" (like the blue-print of a house) for each graph library. No such information is currently available in the documentation associated with each library. From a practical viewpoint, we believe that each such feature model helps in understanding the design of its corresponding library because this model provides a conceptual overview of its library. Then, as a second contribution, in order to highlight the differences between these libraries, we present an integrated feature model of the graph domain and explain how each library 'fits' in it. Though comparing libraries (e.g., with respect to requirements, variability, etc.) lies beyond the scope of this thesis, we hint at the usefulness of our feature models by using them to sketch a comparison between the libraries. 


\section{Contents}

1. Introduction

1.1 The Context

1.2 The Problem

1.3 Potential Applicability

1.4 Contributions and Thesis Organization

2. Background

2.1 A Brief Introduction to Feature Modeling

2.2 C\&E's work on lists

2.3 Graph Libraries

3. Modeling the Individual Libraries

3.1 Graph Template Library from German (GTL-G)

3.2 Graph Template Library form Russia (GTL-R)

3.3 Boost Graph Library (BGL)

3.4 LEDA

3.5 Library comparison

4. The Integrated Model

4.1 About integration

4.2 GTL-G revisited

4.3 GTL-R revisited

4.4 BGL revisited

4.5 LEDA revisited

4.6 Library selection

4.7 Prototyping a Generator

5. Looking for Tests

5.1 State-based testing

5.2 Decision trees

5.3 Considering requirements

5.4 Scenarios

6. Conclusions

6.1 Recapitulation

6.2 Future work

7. References 


\section{List of figures}

2-1 Feature diagram of a simple car

2-2 Feature diagram of a simple list container [11]

3-1 Feature diagram - GTL-G

3-2.a Feature diagram - GTL-R

3-2.b Sub-feature CGraphEdgeArray

3-2.c Sub-feature CGraphAdjList

3-2.d Sub-feature CGraphAjdMatrix

3-3.a Feature diagram - BGL

3-3.b Sub-feature-adjacency_list in BGL

3-3.c Sub-feature - adjacency_matrix in BGL

3-4.a Feature diagram - LEDA

3-4.b Sub-feature - Graph representation in LEDA

4-1 Feature diagram -- Graph from textbooks

4-2 Feature diagram -- Graph from textbooks (2)

4-3.a Feature diagram - Graph

4-3.b Sub-feature - Graph Representation

4-4 Feature diagram - GTL-G after integration

4-5.a Feature diagram - GTL-R after integration

4-5.b Sub-feature - edge array under representation in GTL-R

4-5.c Sub-feature - adjacency list under representation in GTL-R

4-5.d Sub-feature - adjacency matrix under representation in GTL-R

4-6.a Feature diagram - BGL after integration

4-6.b Sub-feature - adjacency list under representation in BGL

4-6.c Sub-feature - adjacency matrix under representation in BGL 
4-7.a Feature diagram - LEDA after integration

4-7.b Sub-feature - graph under representation in LEDA

4-7.c Sub-feature - parameterized graph under representation in LEDA

4-7.d Sub-feature - undirected graph under representation in LEDA

4-7.e Sub-feature - parameterized ugraph under representation in LEDA

5-1 Decision tree for graph domain

5-2 GRL model for Ownership

5-3 GRL model for container type

5-4 UCM model - graph selection

5-5 UCM model - GTL-G

5-6 UCM model - GTL-R

5-7 UCM model - BGL

5-8 UCM model - LEDA 


\section{List of tables}

2-1 Complexity comparison for graph representations

3-1 library comparison

4-1 Feature summary

4-2 Main Variation points

4-3 NFR comparison

5-1 Categories for getNextElement( )

5-2 Categories and Choices for getNextElement( )

5-3 impacts of variation points on performance and modifiability 


\section{Introduction}

\subsection{The Context}

A current trend in software engineering is reuse: reuse of analysis, reuse of design, reuse of code, and reuse of applications. Software reuse is driven by the desire to reduce software development costs and to leverage existing high quality artifacts. Meanwhile, stakeholders are demanding specialized software that fits their specific needs. Software must therefore be easily customizable. This creates conflicting forces on software development: reusing existing software artifacts while creating customized high-quality software that meets the specific needs of its stakeholders. Recent developments in software engineering have attempted to address various aspects of this issue. [24]

Domain driven software development methods, such as software product line engineering $[20,22,25,26]$, system-family engineering $[27,28]$, or generative programming [11], all promote a generative approach to software production and reuse. More specifically, these approaches address variability in a family of systems. This family forms a domain ${ }^{1}$. Modeling software variability facilitates software reuse [e.g., 11, 21, 22]: a highly flexible generic architecture and a set of domain components can be reused in order to produce a new software product within the given family. Given that except for small, if not trivial systems, it will generally be impractical to enumerate all possible systems in a family, we need a generative approach to the capturing of variability, one that implicitly specifies family members as valid possible variations. In order to achieve this, the use of

\footnotetext{
1 The notions of a 'domain' and of a 'family' are used interchangeably in the literature [e.g., 11 ] and in this work. It could be argued however that 'domain' should be viewed as a more general notion than 'family'.
} 
feature models $[23,25,26,29,30]$ is generally advocated. Here, a feature is a "variation point" [11] and a feature model leads to a grammar of valid and invalid combinations of features, and ultimately to a generator ${ }^{2}$. This generator is then used in the application-engineering process to produce an executable system.

It is important to point out that modifiability is different from variability. Modifiability is the ability for a system to be changeable. It constitutes a non-functional requirement, often referred to as "ease-of-modification", which can be measured (e.g., with respect to the number of hours required on average to implement a particular type of change in an existing system). Conversely, variability is the ability for a system to vary in its functionality across a domain. For example, a security component offers a variable encryption strategy if this component can use distinct encryption strategies. On the other hand, this component is highly modifiable if it is trivial for a designer to modify the set of encryption strategies this component handles. [16] From this viewpoint, variability pertains to a domain, whereas modifiability is specific to a particular application (i.e., a particular member of a family of applications). Indeed, some family members may optimize modifiability at the expense of performance, whereas others (i.e., other variants in the family) may do exactly the opposite. Feature modeling is about capturing variability.

Libraries and frameworks offer significant groupings of reusable components. It is possible to view an individual library as constituting a (rather 'narrow' and thus less desirable) domain of its own since each library typically encapsulates some

\footnotetext{
${ }^{2}$ We refer here to a "generator" as defined in [11] as " a program that takes a higher-level specification of a piece of software and produces its implementation".
} 
variability (e.g., offering different implementations for a general concept. But we also notice that it is quite easy to find groups of libraries pertaining to what looks like a common (more general) domain (e.g., containers [31]). In this dissertation, we consider libraries pertaining to graphs. Graphs are mathematical abstractions that are used very often to solve many types of problems in computer science $[1,2]$. For example, graph theory is omnipresent in problems related to computer networks. Much like the Standard Template Library of $\mathrm{C}++[46], \mathrm{C}++$ graph libraries can be seen as typically consisting of three categories of classes: representational ones (pertaining to the implementation of vertices, edges, and their properties), iterators (addressing the traversal of graphs), and algorithms (concerned with the use of graphs). In this dissertation, we will focus on the first of these categories, namely representational issues for graphs. In other words, the domain we select can be characterized as the "representations for graphs in $\mathrm{C}++$ graph libraries". How to generalize this domain to languages that do not use pointers lies beyond the scope of this work. That is, the conceptualizations we offer in the form of feature diagrams are somewhat language dependent (i.e., subsumed by $\mathrm{C}++$ specific notions such as copy-by-value, versus copy-by-pointer).

\subsection{The Thesis}

In this dissertation, the specific problem we tackle is how to facilitate the reuse of a set of independently developed $\mathrm{C}++$ graph libraries. We are concerned more precisely with library users being offered an alternative to having to study in detail the code of a library before deciding whether or not and how to reuse its components. Our fundamental postulate is that domain engineering does facilitate 
reuse, as extensively argued in the abovementioned literature ${ }^{3}$. Thus, the question we ask is the following: can the notions of domain engineering, and more specifically the technique of feature modeling, be applied to a set of independently developed $\mathrm{C}++$ graph libraries? Our thesis is that they can and we intend to demonstrate this by building feature models addressing the representational aspects of a family of independently developed graph libraries.

Before going any further, we want to address the scientific merit of such a question. We believe the question is of importance and that our answer to it represents a contribution to the state-of-the-art in software engineering. Let us briefly elaborate. First, we observe that no $\mathrm{C}++$ graph library we encountered in our investigation offered any model of any sort: just code and textual documentation that did not address the design of the library but merely captured how to use each class. Yet it is generally accepted [e.g., 36, 40, 45] that models provide an abstraction mechanism of considerable value in, at least, the reuse, maintenance and evolution of code. In other words, in software engineering as in other fields of engineering, the creation of models is generally taken to help in the understanding and use of the modeled entities (motivating, for example, vast modeling research as attested by contributions to the Object Management Group [44]).

Second, we observe that when the documentation of a library includes models, typically the latter take the form of so-called class diagrams [see 42,44$]$. But,

\footnotetext{
${ }^{3}$ In other words, demonstrating that feature modeling does facilitate reuse lies beyond the scope of this thesis. Let us simply remark that this postulate has been supported at length in extensive and well-published research, partly cited in the previous section. Also, we are not claiming other types of models would not be able to facilitate reuse of libraries: we are instead postulating that feature diagrams $d o$, mostly, as explained in the literature, because they explicitly and elegantly capture variability.
} 
domain engineers such as Czarnecki and Eisenecker [11] and Evans [45] remark that feature diagrams used in domain modeling capture variability in a simpler way than class diagrams ${ }^{4}$. And, most importantly, researchers in domain modeling [e.g., 11, 20, 22, 23, 25, 26, 27, 28, 29, 30] argue that understanding and modeling variability in software is necessary for productive software development and, in particular, for efficient software reuse. This is a postulate of our work, we repeat, and it suggests that the feature models we develop are indeed a contribution to the state-of-the-art: where no models existed to simplify the task of reusing the set of $\mathrm{C}++$ graph libraries we selected, now we offer feature models that, we postulate, provide an abstraction level facilitating understanding and reuse, much like a blue-print simplifies the understanding of the construction of a house.

Third, again following Czarnecki and Eisenecker ([11], chapters 2 through 5), it is important to acknowledge that feature models in the literature generally considerably overlap both in terms of visual syntax and semantics. In other words, researchers in domain engineering $[11,45]$ observe the emergence of a common modeling language for domain modeling, in the same way as the Unified Modeling Language [44] has established itself for object-oriented modeling. The emergence of what Evans [45] calls a "ubiquitous language" for domain modeling is important for it helps reducing the idiosyncratic nature of modeling and of interpretation Put another way, it is commonly accepted [40] that modeling, especially object-oriented modeling [42], is inherently heuristic based: proving the existence of a single correct model for one system (let alone for a family of systems!) is essentially impossible. But, having a common modeling language and

\footnotetext{
${ }^{4}$ Czarnecki and Eisenecker [11] contrast at length capturing variability in a class diagram and in a feature model, making eloquently the point that the former is much more cumbersome than the latter.
} 
some shared guidelines for carrying variability analysis contributes to somewhat reducing the idiosyncratic (as opposed to systematic) nature of producing feature models. In this dissertation, following [11], we adopt the widely used syntactic and semantic conventions of FODA (Feature Oriented Domain Analysis) [49] accepting some of its inherent semantic limitations (such as the fact that feature diagrams are trees, not graphs, a restriction discussed at length in $\left.[49,11]^{6}\right)$. For now, we want to emphasize that feature modeling is a form of conceptual modeling [11, Appendix A]: as such, as Corriveau points out [37, chapter 2], the notions of completeness and correctness do not readily apply to it. But, as he adds, addressing a category of problems for which the notions of completeness and correctness are difficult to define does not make their modeling, even if somewhat idiosyncratic, a pointless task: research in Natural Language Understanding (Ibid.) eloquently demonstrates this point, that is, building models, even fairly ad hoc ones, does constitute an improvement of the state-of-the-art over the absence of any sort of model. (Also, it is generally acknowledged that completeness and correctness of code itself remains an open problem [43]).

On the issue of the 'adhocness' of the feature models we propose in this work, we remark that, in our opinion, feature models appear to be neither more nor less ad hoc than other abstraction mechanisms used in object-oriented software engineering. For example, though a plethora of guidelines and 'methods' exist, there is no truly systematic method to create, compare and evaluate UML models

\footnotetext{
${ }^{5}$ whose terminology, syntax and semantics are discussed at length in [49], summarized in [11] section 4.4, and overviewed at the beginning of the next chapter of this dissertation. Other feature modeling notations are also discussed in [11] where we found extensive motivation for the choice of FODA.

${ }^{6}$ In summary, in FODA, feature diagrams are restricted to be trees (i.e., hierarchies) in order to ensure that the semantic relationships between a feature and its subfeatures have a systematic interpretation (using notions of first order logic).
} 
[44]. Our point is that conceptualization and abstraction are inherently idiosyncratic and, in particular, depend on existing knowledge of the modeler [37]. For example, as Shi [39] points out, an expert in distributed algorithms will likely not conceptually integrate a family of algorithms for leader election in a ring the same way a novice who only knows about these algorithms would. And, as previously mentioned, adopting the semantics of FODA [49] at least favors a systematic interpretation of such feature models.

'Adhocness' is also inherent to establishing the boundaries of the domain under consideration namely "representations for graphs in $\mathrm{C}++$ graph libraries". More specifically, we must briefly motivate of why we limit the discussion in the rest of this dissertation to four specific libraries when, in fact, other commercial $\mathrm{C}++$ graph libraries exist. Put simply, we restricted ourselves to public domain (free!) ones that advertised themselves as 'comprehensive' libraries with respect to the graph representations they supported. Let us explain. Though in theory graphs can be supported by a multitude of representations, in practice, as explained in the next chapter, three categories of representations are generally used [1,2,3] (namely adjacency list, adjacency matrix and edge list). Considerable variability exists in each of these categories (as will illustrated in chapter 3). This is a priori knowledge (often called domain expertise) we relied on in initially surveying the code and documentation of the libraries we considered: we selected readily accessible libraries that offered one or more of these representational approaches. In other words, the domain is constrained to graph representations varying between and/or within these three categories. Deciding whether it could be desirable to generalize this domain and investigating how this could be done, are 
issues that lie beyond the scope of this work. Let us simply remark that such "domain evolution" is an open problem in domain engineering [11].

Still considering the scientific merit of our work, the analogy to UML suggests we now turn our attention to the 'method' used to create feature diagrams. As previously mentioned, a multitude of methods have been published for object-oriented modeling. But even for widely adopted high-level abstraction models such as use-cases [42], it is commonly accepted that creation is heuristic-based [47]: not only will different modelers produce different models, but little exists in terms of systematic comparison and evaluation mechanisms. And this observation is even more so relevant to more detailed models in which the number of factors that can vary increases dramatically. The point to be grasped is that methods to produce models (including feature models) in a systematic and reproducible fashion still typically elude object-oriented software engineering $[42$, 43]. Acknowledging this fact, we can however briefly summarize here how one goes about building a feature model for a domain:

1. identify domain concepts $[42,47,48]$. For graphs: vertices and edges (as discussed in chapter 3)

2. identify variation points [11]: given a certain perspective (e.g., representational concerns), which concepts can have different instantiations. For example, is there one or several ways of representing a vertex, of organizing its properties, of traversing a graph? Such concepts constitute "variation points" captured as features in a feature diagram. Their possible instantiations are captured as subfeatures of these features 
(see next chapter as well as [11]). Features and subfeatures are organized into a single hierarchy.

In addition to these general guidelines, in our specific work, we have adopted two additional intuitive heuristics based on the study of the code of the libraries at hand:

1. When one can establish a subtyping ${ }^{7}$ [42] relationship between a parent class and its subclasses, the parent class must be investigated as a potential variation point.

2. Each time a template is used, one must ask whether or not this template does correspond to a variation point.

The point to be grasped is that, to the best of our knowledge, beyond these intuitive guidelines, little exists for facilitating (if not making more systematic) the recognition of features. But it is important to repeat that the creation of feature models, even ad hoc ones, does constitute an improvement of the state-of-the-art over the absence of any sort of model for the graph libraries we consider in this dissertation.

\subsection{Potential Applicability}

Our thesis is that feature modeling can be applied to a set of independently developed $\mathrm{C}++$ graph libraries. Our work rests on the postulate that feature models facilitate reuse. Before elaborating on the contributions we contend this work makes to the state-of-the-art, we want to elaborate on the consequences of our

\footnotetext{
${ }^{7}$ Subtyping : a subclass must be able to substitute transparently, with respect to behavior, for its parent class.
} 
postulate. More specifically, we want to hint at the potential applicability and benefits of work. And since we are speculating on such issues and thus will express them in terms of beliefs, as opposed to claims. Our purpose here is to provide additional motivation for the work we develop in the rest of this dissertation.

Belief 1: faced with the documentation and code of a library, we believe the creation of a feature model improves the understandability (and thus the reusability) of the library. It is well accepted in software engineering that code is hard to understand and, generally the main source of software maintenance problems $[36,42,43]$. And documentation is more than often too detailed, and usually extremely code-centric. Within object-oriented software engineering, such observations have lead to the emergence of proposals such as the Model Driven Architecture of the Object Management Group [44]. In a nutshell, the proponents of such initiatives contend that models must become the central artifacts of the software development process. But demonstrating that a particular model improves something as hard to evaluate as understandability is quite a challenge: psychological studies measuring learning curve, as well as reuse strategies, of a wide spectrum of developers would likely be required. This is not what this dissertation is about.

More modestly, we simply want to enable such eventual research by first developing here feature models (whose usage could then be studied in such psychological experiments). 
Now consider, not a user 'forced' to use a particular library, but rather the familiar industrial task of having to select one from several libraries relevant to a specific project. Let's call this the library selection problem. How does one go about achieving this task in an objective and systematic way? It is unreasonable to expect every designer to be an expert on each library at hand. Conversely, an expert, especially one who would have built a feature model for a library in the domain, can be expected to be able to build a feature model for each library to consider and, most importantly, an integrated feature model, that is, one that provides a unified conceptual framework for understanding and possibly comparing these libraries.

Belief 2: faced with the library selection problem, developing in an integrated feature model across these libraries may be a worthy investment. The value of this investment will likely be proportional to the number of users who will be faced with the library selection problem ${ }^{8}$. And it is important to understand that each user typically brings a specific set of requirements and constraints to the problem. Thus, the ability of the integrated feature model to capture variability across the domain (and to relate this variability to functional and non-functional requirements) is crucial: each act of selection is unique and must consider the entire domain. We believe thinking of a common domain for these libraries, doing a feature diagram for this domain and then documenting how each of the libraries at hand "fits in" this integrated model constitutes a good solution to the library selection problem. Again, demonstrating the "goodness" of this approach is not the intention of this

\footnotetext{
${ }^{8}$ While domain engineering has the drawback of atypical significant upfront investment of time, we simply observe that this investment may be leveraged if one considers the amount of time a feature model (as a road-map or blue-print of a library) potentially saves other designers who use it. Such considerations however lie beyond the scope of this thesis.
} 
dissertation (and is probably, again, quite hard to achieve due to the paucity of other approaches to compare with, as well as due to the semantic elusiveness of the notion of 'goodness'). Instead, our mandate here is to investigate whether the integration of the conceptualizations (i.e., feature models) of several large industrial libraries (for graphs) is feasible within the bounds of a single feature model. And indeed we do present such an integrated feature model and briefly explain how we obtained it. It will be to others to investigate the usefulness of this model; we merely demonstrate it can be done and suggest how to go about it. In the same spirit, we believe that the models we propose here will also be useful in providing data for experiments eventually created to address a final conviction of our research group, namely:

Belief 3: an integrated feature model across of domain that includes several libraries likely enables significant productivity gains with respect to the production and reuse of tests across these libraries.

We observe that little is currently done with respect to quality engineering in the context of domain modeling. Very few, if any, domain-driven approaches explicitly consider quality, and few acknowledge that the systems that are generated must be tested ${ }^{9}$. The goal of our research group's work is to make quality a pervasive concern in domain-driven engineering. We believe this standpoint has two advantages. First, it promotes the role and importance of

${ }^{9}$ Quite on the contrary, it is often the case that researchers in domain engineering see no point in testing an application produced by the generator that corresponds to a feature model. Such a naive viewpoint forgets that $i$ ) there is typically a significant conceptual gap between rough feature models and actual generators and ii) even assuming a fault-free generation process still does not eliminate the task of verifying the generated family member against the set of requirements that were used to select its features. In other words, one must check that the application that is obtained does indeed satisfy the requirements that guided the selection of features of this application. 
quality engineering early in the process, a highly desirable characteristic for software development [17]. Second, we believe bringing quality into domain-driven development enables test generation across a family of systems and improves the reuse of tests across this family [24]. In this dissertation, we very briefly address, in the future work section, the issue of quality engineering (given this is the central theme of other dissertations in our research group). More precisely, we will merely elude to how tests could be generated from the additional models we suggest. This investigation, we repeat, is carried in other dissertations within our research group.

\subsection{Contributions and Thesis Organization}

Having discussed, our thesis, the postulate that subsumes it, and its motivations via its possible applicability, let use summarize our achievements. We believe this dissertation makes two contributions:

- Contribution 1: In chapter 3, we develop a feature model for each graph library we select. Such a blueprint of each library did not exist. Also, for each library we give an initial set of lessons learnt from modeling this library in isolation. We emphasize these are lessons beyond what is in the original documentation of the library. Each of these libraries, as well as the basics of feature modeling, is introduced in chapter 2.

- Contribution 2: In chapter 4 we present an integrated feature model for the domain we have selected, and a reviewed feature diagram for each library (to explain how it fits in the domain). This is our major contribution in our opinion since it provides, to the best of our knowledge, the first time the library selection 
problem is addressed by explaining the differences between the libraries WITHIN a common conceptual framework (provided by the integrated feature diagram). Our solution takes the form of two comparison tables, which we hope will be of immediate interest to potential users of the libraries we studied.

With respect to our first contribution, we emphasize that each road map requires that its creator study in detail the documentation and code of the different libraries that form the domain. Deep knowledge of the domain is required here and it is probably unrealistic to expect all library users to acquire such a level of understanding. We believe this point reinforces the likely usefulness of the proposed models, especially given the fact that the four libraries we studied correspond to approximately 16 megabytes of code and almost 900 files!

Finally, as a minor contribution, we summarize in chapter 5 the generator we implemented out of the integrated feature model used in chapter 4 . This generator was developed strictly for the purpose of investigating how feasible it was. Comparing the classes its generates to those of the existing libraries, as well as any other form of evaluation of this generator, lie beyond the scope of this work.

The dissertation concludes with a brief summary, in chapter 6 , of our contributions and a description of some directions taken as future work in our research group. 


\section{Background}

\subsection{A Brief Introduction to Feature Modeling}

Recently, several authors [e.g., 11, 20,21, 22] have advocated improving software reuse by means of a generative approach to the modeling and programming of a family of systems. Recall that except for small if not trivial systems, it will generally be impractical to enumerate all possible systems in such a family. Instead, a highly flexible architecture and a set of reusable components are to be designed to maximize variability in order to maximize the ability to generate customized family members. This family forms a domain, which is usually represented using a feature model $[11,23]$. A feature is generally described in as a logical unit of behavior that is specified by a set of functional and quality of service requirements. In most models, features are classified into these categories:

- Mandatory - supported by all systems in the family. (black dot)

- Optional - supported by some systems in the family. (white dot)

- Alternative - alternatives for each other. (arc between them)

- Or - any subset. (filled arc between them)

A feature value is a feature that is not composed of any other features. A feature model must capture a grammar of valid and invalid combinations of features, and ultimately a generator. In this work, we adopt the feature modeling notation used by Czarnecki and Eisenecker [11]. 
First we use a simple example from [11] to illustrate the feature modeling notation.

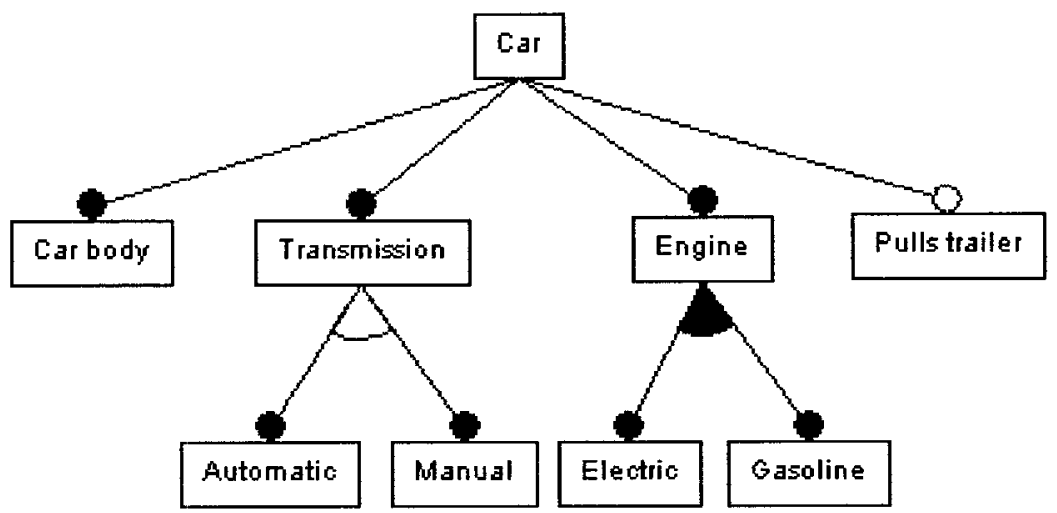

Figure 2-1 Feature diagram of a simple car

The car consists of a car body, transmission, and an engine. The transmission may be either manual or automatic. Furthermore, the car may have a gasoline engine or an electric engine or both. Finally, the car may pull a trailer. Figure 2-1 shows a feature diagram of a simple car. Feature Car has three mandatory sub-features: car body, transmission, and engine, and one optional feature value: Pulls trailer. The Transmission has two alternative feature values: Automatic and Manual. The Engine has two or feature values: Electric and Gasoline.

Much more "sophisticated" notations have been proposed in the context of family engineering but our contribution does not rest on the adoption of any particular notation for feature modeling. And we also want to emphasize that we make no attempt in this word at addressing the limitations shared by all feature modeling techniques (e.g., the gap between requirements and feature models, the oversimplification of how to capture feature interactions, the gap between a feature model and its corresponding generator). Suffice it to say here that other 
members of our research group are tackling these issues: each is indeed worthy of its own thesis!

\subsection{C\&E's work on lists}

In [11], a list container, namely single linked lists, is used as an example to demonstrate the basic steps to design and implement a generative domain model. These steps include domain analysis, domain design, component implementation and finally a generator. Let us first take a look at the feature diagram in [11]:

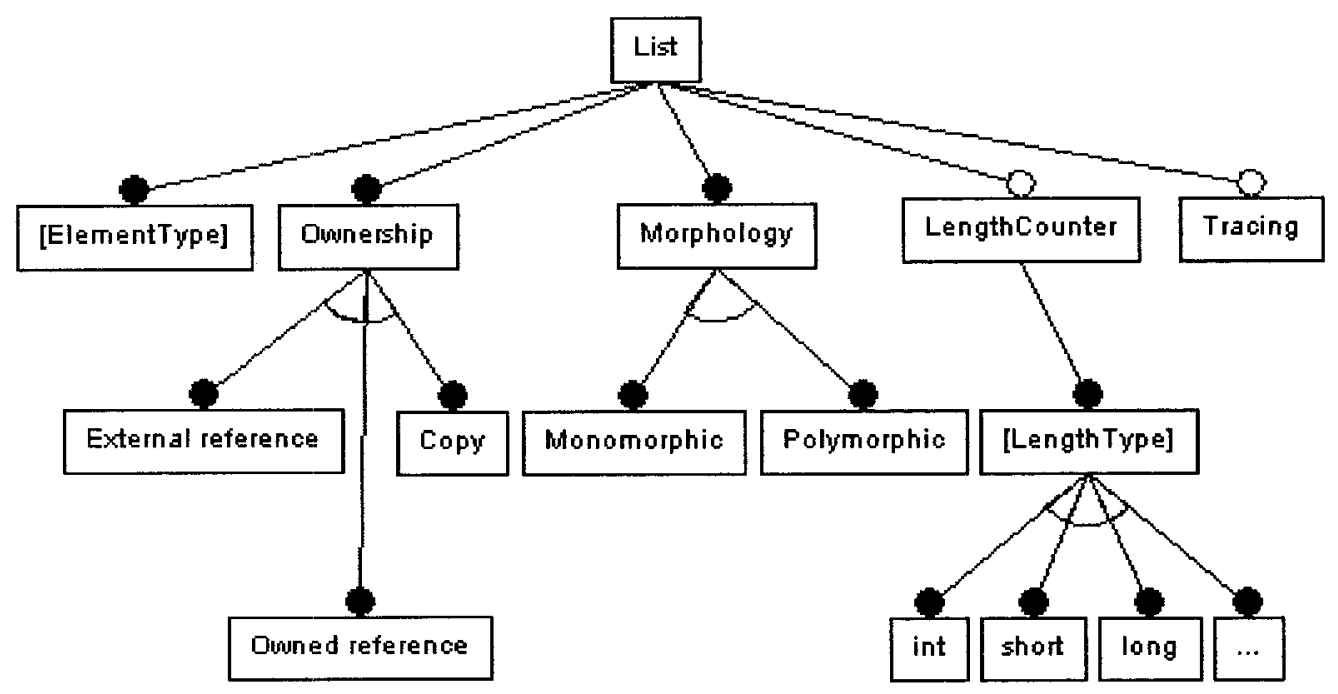

Figure 2-2 Feature diagram of a simple list container [11]

The important property of a feature diagram is that it allows you to model variability without having to commit to a particular implementation mechanism, such as inheritance, aggregation, templates, or \#ifdef-directives [11]. Without counting ElementType and LengthType (for which [] are used to signal these features can change at run time), the diagram in Figure 2-2 describes 24 list variants (three different ownership kinds, two morphology kinds, an optional length counter, and an optional tracing, i.e., $3 * 2 * 2 * 2=24$ ). 
From this diagram, a list generator is developed in [11]. Let us take a look at this generator to assess the correspondence that ought to exist from a feature diagram to its corresponding generator.

First define the feature vocabulary for specifying lists.

Features defined in Figure 2-2 are represented by using enumeration types

enum Ownership \{ext_ref,own_ref, cp\};

enum Morphology \{mono, poly\};

enum CounterFlag \{with_counter, no_counter\};

enum TracingFlag \{with_tracing, no_tracing\};

The generator uses these features as template parameters. The parameter list is extendible. By choosing appropriate defaults for the new parameters, existing calls to the generator will still work properly:

template<

$$
\begin{aligned}
& \text { class ElementType, } \\
& \text { Ownership ownership }=c p, \\
& \text { Morphology morphology }=\text { mono, } \\
& \text { CounterFlag counterFlag }=\text { no_counter }, \\
& \text { TracingFlag tracingFlag }=\text { no_tracing, } \\
& \text { class } \quad \text { LengthType_ }=\text { int }
\end{aligned}
$$$$
>
$$

class LIST_GENERATOR

f

public:

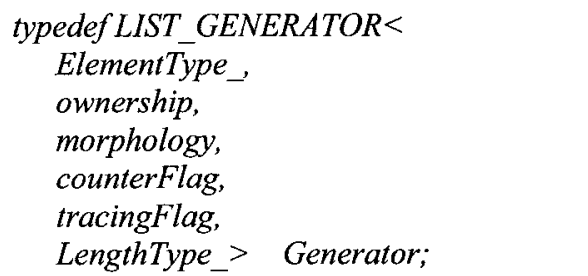

private:

$$
\begin{array}{ll}
\text { enum }\{ & =\text { ownership }==c p, \\
\text { isCopy } & =\text { morphology }==\text { mono, } \\
\text { isOwnRef }=\text { ownership }==\text { own_ref, } & \\
\text { isMono } & =\text { counterFlag }==\text { with_counter }, \\
\text { hasCounter } & =\text { tracingFlag }==\text { with_tracing }\} ; \\
\text { doesTracing } & =\text { tracing }
\end{array}
$$

//Choosing destroyer according to the value of Ownership

$$
\begin{aligned}
& \text { typedef } \\
& \text { IF }<\text { is Copy } \| \text { is OwnRef, } \\
& \text { ElementDestroyer }<\text { ElementType }>, \\
& \text { EmptyDestroyer }<\text { ElementType_> } \\
& >\text { : RET Destroyer_; }
\end{aligned}
$$

//Choosing typechecker according to the value of Morphology

typedef

IF $<$ is Mono,

DynamicTypeChecker <ElementType_>,

EmptyTypeChecker<ElementType_> 
>::RET TypeChecker_;

//Choosing copier according to the value of Ownership and Morphology typedef $I F<$ isCopy,

IF $<$ isMono,

Monomorphic Copier $<$ ElementType $>$,

PolymorphicCopier $<$ ElementType_> $>:: R E T$,

EmptyCopier $<$ ElementType $>$

$>: \because R E T$ Copier_;

typedef

IF $<$ is Copy,

const ElementType,

ElementType

$>: \because$ RET SetHeadElementType;

typedef PtrList $<$ Generator $>$ List;

//If LengthCounter is selected ...

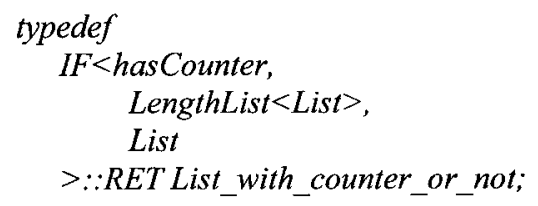

//If Tracing is selected ...

typedef

$I F<$ doesTracing,

TracedList $<$ List_with_counter_or_not $>$,

List_with_counter_or_not

public:

$>: \because R E T \bar{L}$ ist_with_tracing_or_not;

typedef List_with_tracing_or_not RET;

//Config makes all information retrievable outside the generator.

struct Config

\{

$\begin{array}{lc}\text { typedef ElementType } & \text { ElementType; } \\ \text { typedef SetHeadElementType } & \text { SetHeadElementType; } \\ \text { typedef Destroyer } & \text { Destroyer; } \\ \text { typedef TypeChecker } & \text { TypeChecker; } \\ \text { typedef Copier } & \text { Copier; } \\ \text { typedef LengthType } & \text { LengthType; } \\ \text { typedef RET } & \text { ReturnType; }\end{array}$

;

The point we want to make is two-fold. First, it should be obvious that the

generator proceeds directly from the feature model. The point to be grasped is that there is no family engineering without a feature model first. Second, the generator implements a multitude of combination rules that are not explicitly captured in the 
feature model. Our research group has made specific contributions in the area of feature modeling to attempt to have such rules "percolate up" into feature models per se. But, in our work we repeat, we will limit ourselves to use the feature modeling notation proposed in [11].

\subsection{Graph Libraries}

Graphs are well known and much-studied mathematical entities. Graphs are discrete structures consisting of vertices, and edges that connect these vertices. [1] Edges can be directed or not, and weighted or not. A graph should provide a data structure to store elements, as well as methods (or equivalently, procedures or member functions) to search for such elements. Searching a graph means systematically following the edges of the graph so as to visit the vertices of the graph. A multitude of other algorithms exists for graphs. Also, there are three standard ways to implement a graph $G=(V, E)^{10}$ : as a collection of adjacency lists, or as an adjacency matrix, or as set (or array) of edges. Adjacency-list and adjacency-matrix are mostly used. A multitude of other representations are possible.

The adjacency-list representation is usually preferred because it provides a compact way to represent sparse graphs - those for which $|\mathrm{E}|$ is much less than $|\mathrm{V}|^{2}$. [2] An adjacency-matrix representation may be preferred, however, when the graph is dense -- $|\mathrm{E}|$ is close to $|\mathrm{V}|^{2}$ - or when we need to be able to tell quickly if there is an edge connecting two given vertices [2]. The Edge-array representation is preferred when a graph is sparse and all operations are related to edges.

\footnotetext{
${ }^{10}$ E represents the set of Edges and V the set of vertices, thus $|\mathrm{E}|$ is the number of edges.
} 
The vertices in adjacency-list are typically stored in an arbitrary order [2]. One advantage of adjacency-list is that it can readily be adapted to represent weighted graphs, that is, graphs for which each edge has an associated weight, typically given by a weight function $w: E \rightarrow R$. For example, let $G=(V, E)$ be a weighted graph with weight function $w$. The weight $w(u, v)$ of the edge $(u, v) \in E$ is simply stored with vertex $v$ in $u^{\text {'s }} \mathrm{s}$ adjacency list. The adjacency-list representation is quite robust in that it can be modified to support many other graph variants [2]. A potential disadvantage of the adjacency-list representation is that there is no quicker way to determine if a given edge $(u, v)$ is present in the graph than to search for $\mathrm{v}$ in the adjacency list $A d j[\mathrm{u}]$. This disadvantage can be remedied by an adjacency-matrix representation of the graph, at the cost of using asymptotically more memory [2].

In the adjacency-matrix representation, vertices are numbered $1,2, \ldots,|\mathrm{V}|$ in some arbitrary manner [2]. The adjacency matrix of a graph requires $\Theta\left(\mathrm{V}^{2}\right)$ memory, independent of the number of edges in the graph. The adjacency matrix is symmetric along the main diagonal. In some applications, it pays to store only the entries on and above the diagonal of the adjacency matrix, thereby cutting the memory needed to store the graph almost in half [2]. Like the adjacency-list representation of a graph, the adjacency-matrix representation can be used for weighted graphs [2]. The weight can be simply stored as the entry. Although the adjacency-list representation is asymptotically at least as efficient as the adjacency-matrix representation, the simplicity of an adjacency matrix may make it preferable when graphs are reasonably small [2]. 
Here is a comparison of complexity for these three representations.

Table 2-1 Complexity comparison for graph representations

\begin{tabular}{|l|l|l|}
\hline & Memory & Edge searching \\
\hline Adjacency-list & $\mathrm{O}(\mathrm{V}+\mathrm{E})$ & $\mathrm{O}(\mathrm{E})$ \\
\hline Adjacency-matrix & $\Theta\left(\mathrm{V}^{2}\right)$ & $\mathrm{O}(1)$ \\
\hline Edge-array & $\mathrm{O}(\mathrm{E})$ & $\mathrm{O}(\mathrm{E})$ \\
\hline
\end{tabular}

The Adjacency-matrix has the largest memory complexity but the search complexity is the smallest. Edge array uses the least memory space but takes more time to search. Adjacency-list has the search complexity related to the number of edges and vertices, but uses more memory space than Edge array.

Given this short introduction to graphs and their implementations, let us now turn our attention to libraries per se. In this work, we will consider the following graph libraries:

- The Graph Template Library from Germany (GTL-G) (310 files, 3.05MB) [3]

- The Graph Template Library from Russia (GTL-R) (186 files, 11.4MB) [5]

- The LEDA Graph Library (293 files, 2.21MB) [7]

- The Boost Graph Library (BGL) (74 files, 789KB) [6]

This selection was determined, first and foremost, with respect to public domain availability.

LEDA ("Library of Efficient Data types and Algorithms") has been developing since 1989 under the efforts of a group at the Max Planck Institute in Saarbrucken Germany [18]. It is a library of the data types and algorithms for combinatorial 
and geometric computing [4]. It provides a sizable collection of data types and algorithms including: stacks, queues, lists, sets, dictionaries, ordered sequences, partitions, and priority queues; directed, undirected, and planar graphs, lines, points, and planes; and many algorithms in graph and network theory and computational geometry. Many data types in LEDA are parameterized, including some graph implementations. LEDA contains a data type graph. It offers the standard iterations, such as "for all nodes $v$ of a graph $G$ do" (written forall_nodes $(v, G))$ ) or "for all neighbour $w$ of $v$ do" (written forall_adj_nodes $(w, v))$. The data type graph allows one to write programs for graph problems in a form close to the typical textbook presentation. Ultimately, the creators of this library summarize their goal in the equation "Algorithm + LEDA $=$ Program." $[4]$

GTL-G is a graph library from University of Passau, Germany. It is a platform independent and extendible $\mathrm{C}++$ library. At the moment the following configurations are tested: [3]

- Windows XP using Visual Studio .NET

- Linux using gec 3.X

- Solaris using gec 3.x

- Mac OS X 10.2 Jaguar

GTL-G contains the classes needed to work with graphs, nodes and edges and some fundamental algorithms as building blocks for more complex graph algorithms. Further algorithms are under development. [3] 
GTL-R comes from Russia. It was developed by students and scientists of Nizhny Novgorod State University [5]. The library was developed in $\mathrm{C}++$ language as a set of template classes. It is a class library for using graph theory algorithms in applications. The three most popular graph representation models (discussed above) are used. Basic algorithms are implemented for these models; they help performing metric and structural analyses, as well as solving typical problems in the use of graphs in other application [5]. GTL-R has a visual interface for graph and graph algorithms.

Finally, the BGL began its life as the Generic Graph Component Library (GGCL), a software project at the Lab for Scientific Computing (LSC) at the University of Notre Dame. Soon after the Standard Template Library was released, work began at the LSC to apply generic programming to scientific computing. On September 4, 2000 GGCL passed the Boost formal review and became the Boost Graph Library (BGL).[6] The first release of BGL occurred September 27, 2000[6]. The standardized generic interface for traversing graphs it provides is of utmost importance to encourage reuse of graph algorithms and data structures. Part of the Boost Graph Library is an interface for how the structure of a graph can be accessed using a generic interface that hides the details of the graph data structure implementation. This is an "open" interface in the sense that any graph library that implements this interface will be interoperable with the BGL generic algorithms and with other algorithms that also use this interface. BGL provides some general purpose graph classes that conform to this interface, but they are not meant to be the "only" graph classes; there certainly will be other graph classes better for 
certain situations. The main contribution of the BGL is the formulation of its interface [6].

These are four graph libraries we choose to model. They are all written in $\mathrm{C}++$. There are a few other graph libraries in existence, such as the LINK graph library [32] and Stanford's Graphbase [19]. LINK is implemented in C++. Stanford GraphBase is written in CWEB, which is a mix of the C programming language and Knuth's TEX typesetting language [19]. The reason we choose these four libraries is that they cover the most variations of Graph, and other libraries' implementations are similar with one or more libraries we are modeling. That is, in scoping out this dissertation, we invested numerous hours in analyzing libraries and then decided to restrict ourselves to four that would each bring something specific to the domain. In other words, we chose to avoid selecting a library that would be excessively redundant with others. Also, different programming languages may have very different conceptual frameworks. Given the complexity of the $\mathrm{C}++$ libraries, we decided to stick with that language only.

Normally, we approach a library by reading its documentation and code. Let us use the node class in LEDA as an example. First we need find it out. It is in graph_objects0.h. The code looks like this:

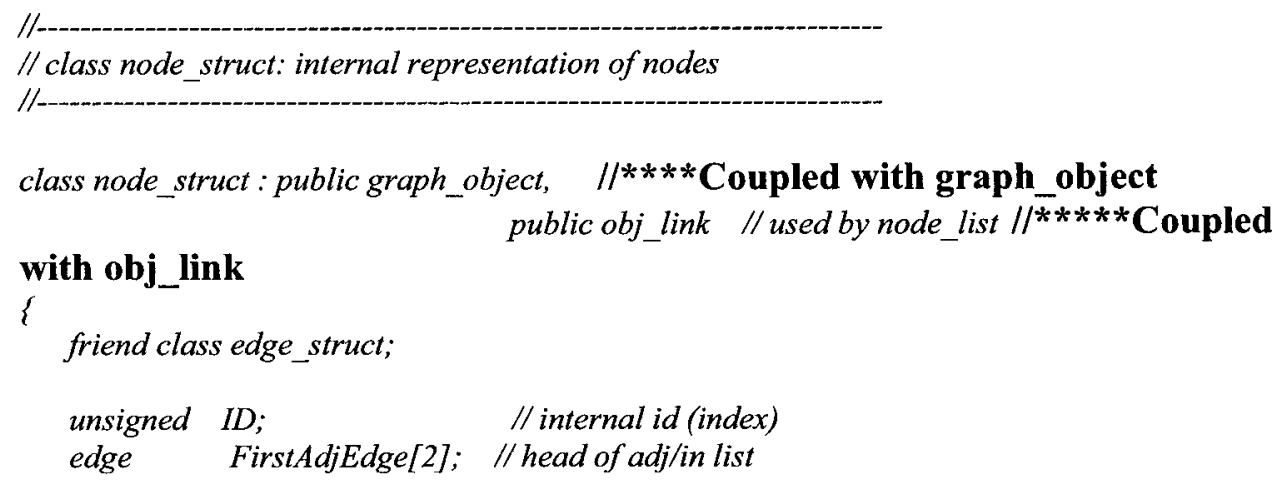




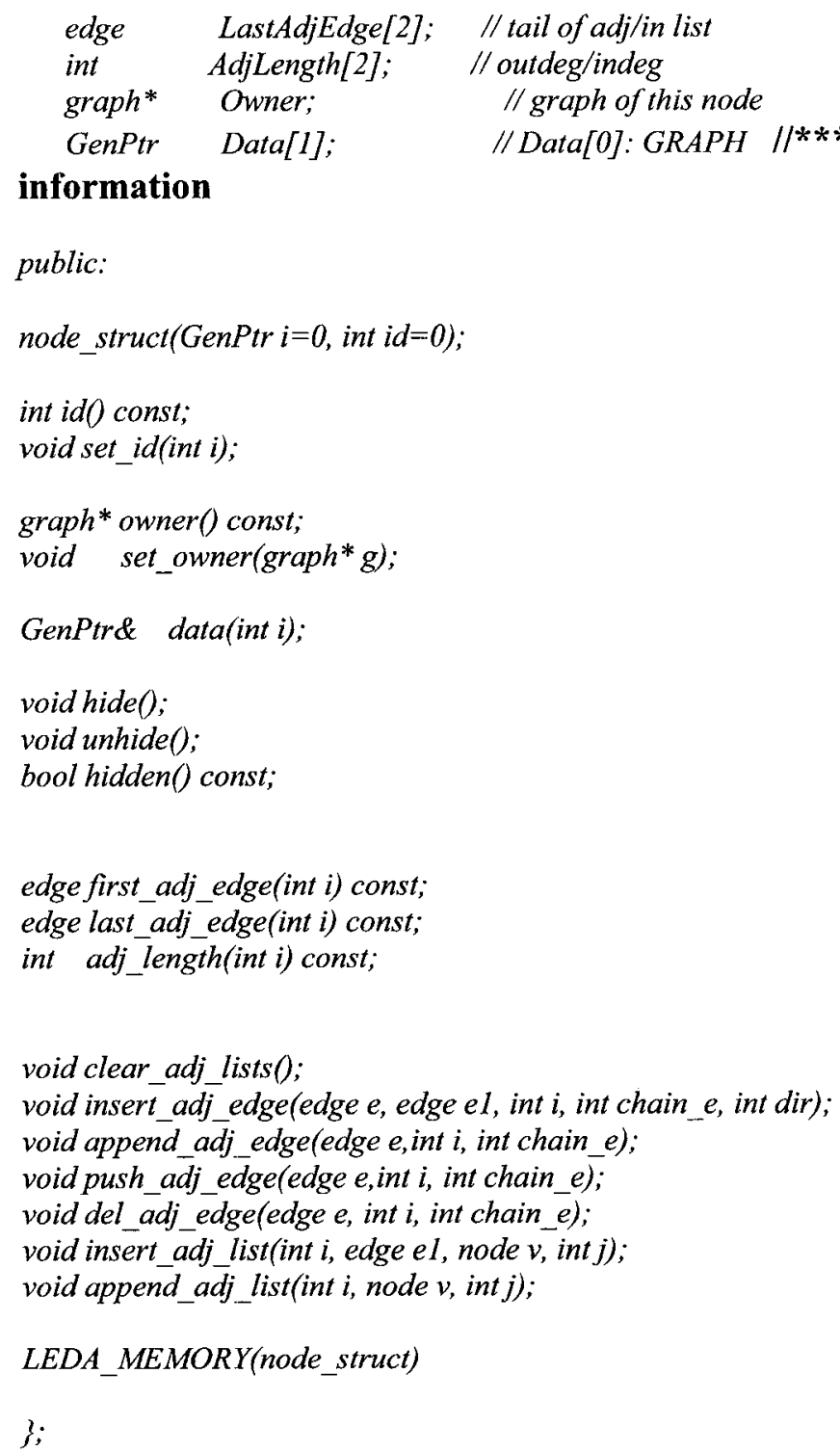

In this simple example, the node is tied to graph_object,obj_link. The node also uses a GenPtr for node data. In order to understand the node class in LEDA, we have to understand all of the above! Such considerations are implementation related details. But without a feature diagram, a user will have to reconstruct such dependencies in her mind in order to have a chance to find and understand such basic information as how a node is represented... Having a feature diagram will, in our opinion, considerably reduce, if not eliminate, the need to "dive into the code" to understand the basics of the library. 
For those who are at the stage of library comparison or selection, this information is also too detailed. The point to be grasped is that some of the conceptual framework we are looking for is actually lost during the development of a library. As mentioned in [11]: The problem with current software engineering is that we usually end up with a concrete software system, but don't know how we got there. Most of the design knowledge is lost, and this makes software maintenance and evolution very difficult and costly to perform.

We believe that investing in constructing feature models may offer an desirable alternative to what Ed Yourdon [36] calls "the marine corps mentality": real men dive into the code (often not even bothering with reading the documentation...) Yourdon makes the point that such code-centric practices largely explain the rate of failure of software projects today, a rate he establishes at above $85 \%$ ! As previously mentioned, measuring the usefulness of feature models lies beyond the scope of this work.

Going back to the creation of the feature diagrams we propose, we emphasize that they do involve "diving into the code" (and documentation!!) but only by an expert, the domain engineer. As the author of this dissertation can attest, domain engineering represents a large investment of time "deciphering" code. Code is included in the rest of this thesis to remind the reader that, behind the (hopefully) intuitive, if not simplistic, appearance of the feature models we propose, there are hundreds of hours spent understanding the libraries we selected!! The contrast between the complexity of the actual code at hand and the simplicity of our feature 
models serves the purpose of constantly reminding the reader that the simplicity of the proposed feature models should not be wrongly conflated with the assumption they are trivial. Quite on the contrary: the simplicity of our results is first and foremost a testimony to the virtues of conceptual abstraction, which is at the root of domain engineering: the more the feature models are understandable, the more we will have succeeded at allowing their user to understand the target libraries without having to go and invest (like we did!) huge amounts of time to understand the details of the libraries.

Also, we must emphasize again that the feature models we propose are not meant to be the only correct ones! Feature modeling, we repeat, rests on conceptual abstraction from code and documentation of libraries. This process constitutes an idiosyncratic act of interpretation [37]: each domain expert may produce different feature models. Thus, much like for design patterns [13], domain engineering will require the conventionalization of domain models: such models will become reusable once and only once several domain engineers will agree that their idiosyncratic conceptualizations have been "melted" in the conventionalized domain model. The point we thus want to make is that we do not claim here that our proposed models are correct (for this notion may not apply!) nor that they are even conventionalized (since we did not find other domain engineers for graph libraries!). It is crucial, however, to understand that, in our opinion, this observation does not diminish in any sort of way our contributions: it is not the exact feature models we obtain but the act of creating these feature models that is being promoted in this work! 
Finally, we observe that our work is in the same spirit as what is called "reverse-engineering" but works at a much more abstract level. The state of the art in reverse engineering aims at extracting component specification from existing code. And, looking at commercial tools such as ROSE [38], we can only express some reservations about the current success of such approaches. Feature modeling is not concerned with components (which partially explains the gap between feature models and the generator, the latter being component-centric). And thus, feature models work at a much higher level of abstraction, one that, most importantly, aims at capturing variability. To the best of our knowledge, variability is totally eliminated from current reverse-engineering approaches for they are rooted in a single-system philosophy. 


\section{Modeling the Individual Libraries}

In this chapter, we present our proposed feature models developed independently for each library (although, we confess, we got a good idea of which concepts we would find across libraries from textbooks that indeed present an abstract viewpoint of the graph domain). All information captured in these models is gathered from documentation and code of each library. We insist that we don't believe the proposed sets of features are complete and sufficient with respect to the intricacies of each library.

\subsection{Graph Template Library from Germany (GTL-G) :}

GTL-G is presented as an extension of the Standard Template Library (STL) for graphs and fundamental graph algorithms [3]. It has been designed and programmed following the guidelines of STL [3]. Adjacency list is the only graph representation in it.

Its html version of documentation is available from [3]. But the documentation is all about implementation details. It lacks the overall conceptual design of the library. We can easily find out a class, the interface of an object and well documented code from the documentation. But there is no single part in it talking about its design decisions; what it is good at and what is not good at. All this information is hidden in the code, that is, "behind" the documentation. A potential user has to go through all the documentation to decide whether this library can meet his requirements. 
Its coding style, we repeat, is similar to STL: fairly simple and easy to understand.

It uses STL::list to store items, uses STL::iterator to search the graph. STL::list provides fast insertion anywhere, but provides only sequential access [33].

Iterators for lists in STL are bidirectional, they can be moved forward or backward, and can be used to read or write the elements of the sequence [Ibid.]. Moving an iterator from one part of the sequence to another takes time proportional to the number of elements between the two [Ibid.].

In order to illustrate the challenge of understanding this albeit well-written code, we first consider the class edge. The definition is in the file edge.h. Here are the declarations:

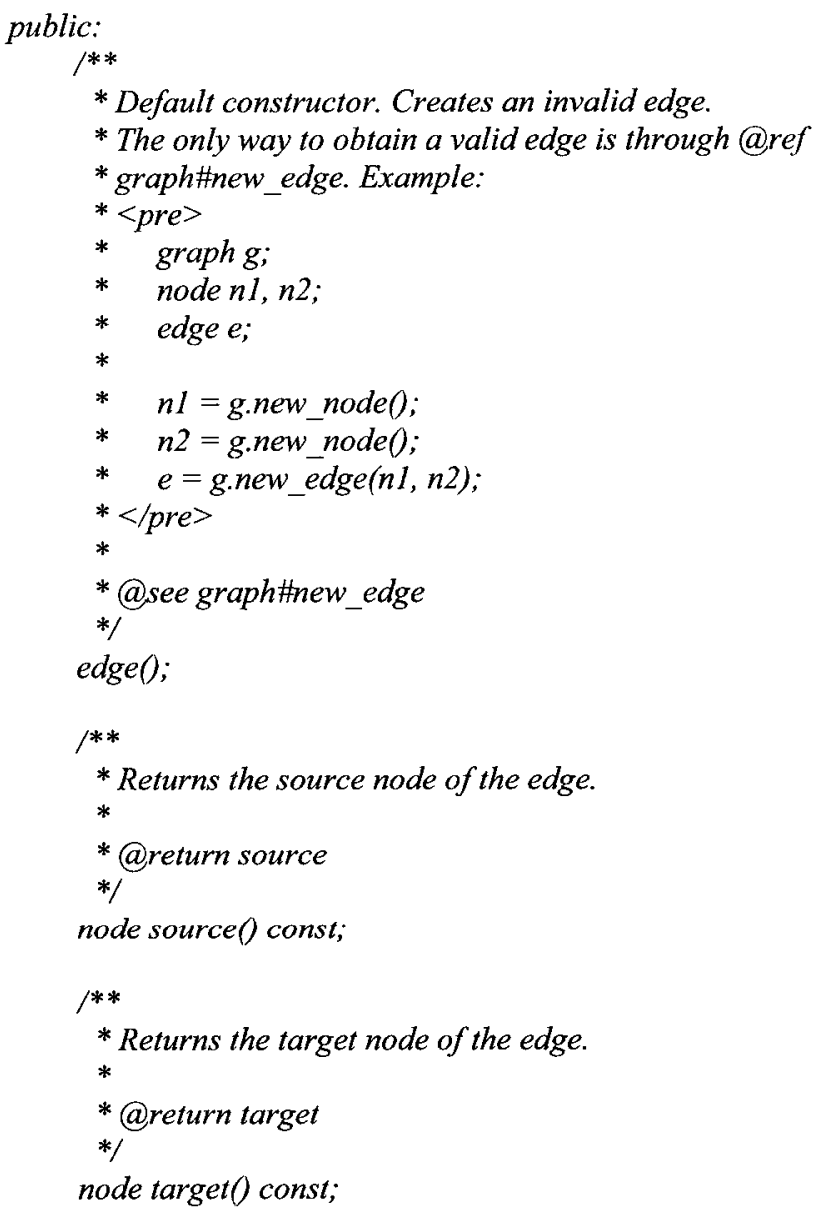


* Changes the direction of this edge.

*/

void reverse 0 ;

/**

${ }^{*}$ Makes $<$ code $>n</$ code $>$ the source of this edge. Takes $O(1)$ time.

$*$

*@param $<$ code $>n</$ code $>$ new source

*/

void change_source (node n);

/**

* Makes $<$ code $>n</$ code $>$ the target of this edge. Takes $O(1)$ time.

*@param $<$ code $>n</$ code $>$ new target

*)

void change target (node $n$ );

$/ * *$

* Returns the node opposite to $<$ code $>n</$ code $>$ referring to

* this edge.

*@param $<$ code $>n</$ code $>$ a node incident to this edge

*/

const node\& opposite(node n) const;

/**

*@internal

*)

list $<$ node $>$ sources() const;

$/ * *$

*@internal

*/

list $<$ node $>$ targets() const;

/**

*@internal

*/

int id() const;

$/ * *$

* Returns true iff node is hidden.

* @return true iff node is hidden.

*@see graphthide_edge

*@see graph\#restore_edge

*)

bool is_hidden 0 const;

$/ /===== \pm================$ Implementation

private:

edge_data *data;

void remove from(int where) const; // $0=$ sources, $1==$ targets

friend class graph;

friend class node; 
GTL_EXTERN friend bool operator $==($ edge, edge $)$;

GTL EXTERN friend bool operator! $=($ edge, edge $)$;

GTL_EXTERN friend bool operator $<$ (edge, edge);

\}

GTL EXTERN friend ostream\& operator $<<($ ostream\& os, const edge\& e);

_GTL_END_NAMESPACE

\#endif // GTL EDGE H

The code, we insist, is well documented. For example, it gives an example to show how to use a default constructor. Another example: the complexity of some methods is also documented. Now let us look at an example of a definition of a member function:

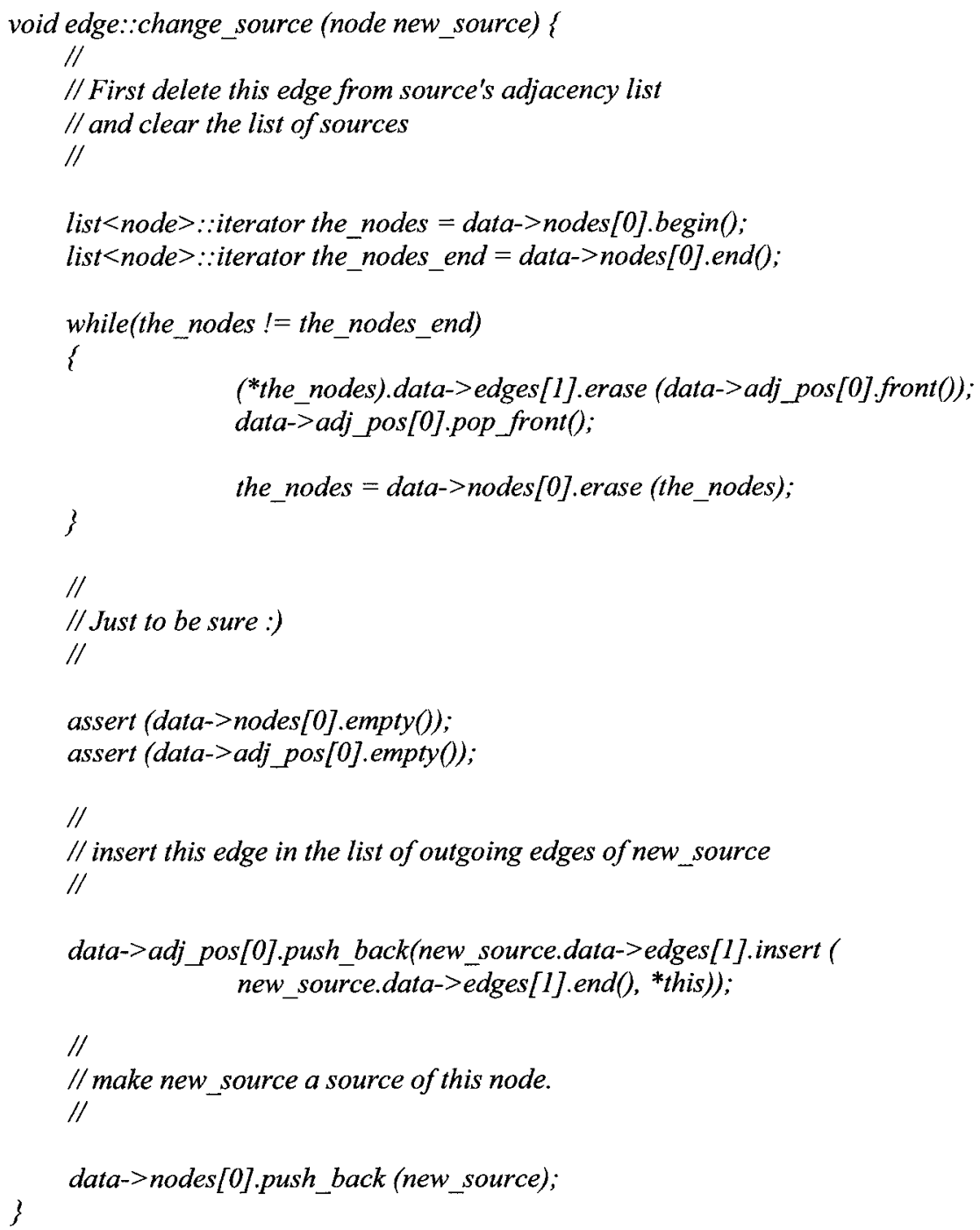


This method uses class edge_data. In order to understand this method, we have to go through class edge_data...

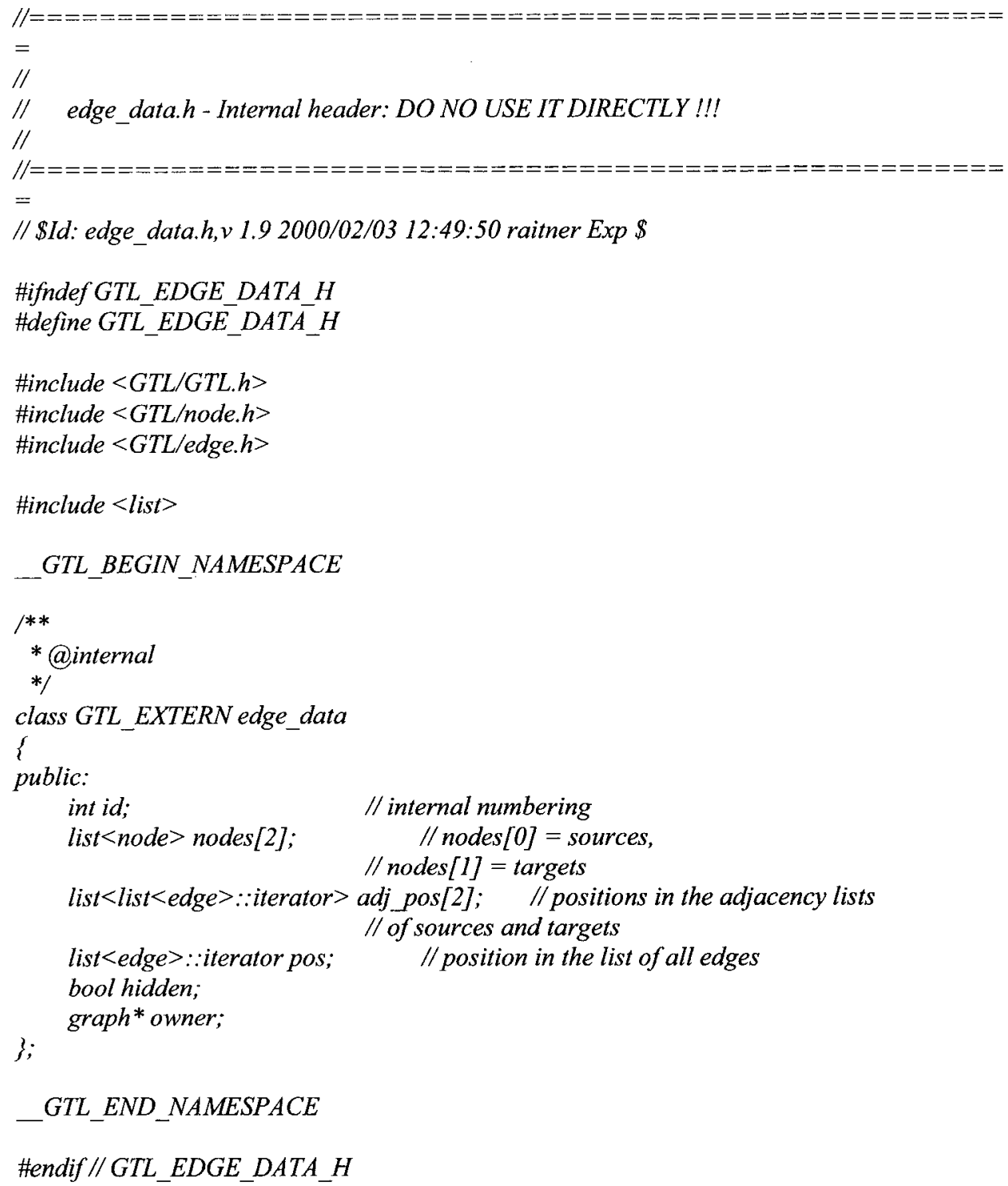

The documentation in edge_data is not so detailed; how it works is not easily understandable... In order to understand edge_data, we have to go back to the edge class, and even to the graph class. It is through such arduous deciphering of the code that features come to mind! And one can never be sure that all original design decisions have been captured. It is like the library designers built a house 
but then threw away the blue prints. Our job is to redraw as much as possible of the blue prints by surveying the house...

GTL-G maybe the simplest one among all libraries we selected and its documentation is detailed, but we still needed much time to go through the code. And through this exercise, we not only "discovered" features but also raised some questions with respect to the original requirements. For example: why GTL-G implemented its edge class the way it is, i.e. what's the purpose and what are the consequences of such a decision. Only through domain analysis on feature models augmented with requirements do we believe such questions can be answered. For now, questions pertaining to requirements lie well beyond the scope of our work.

Here is our proposed feature diagram for GTL-G:

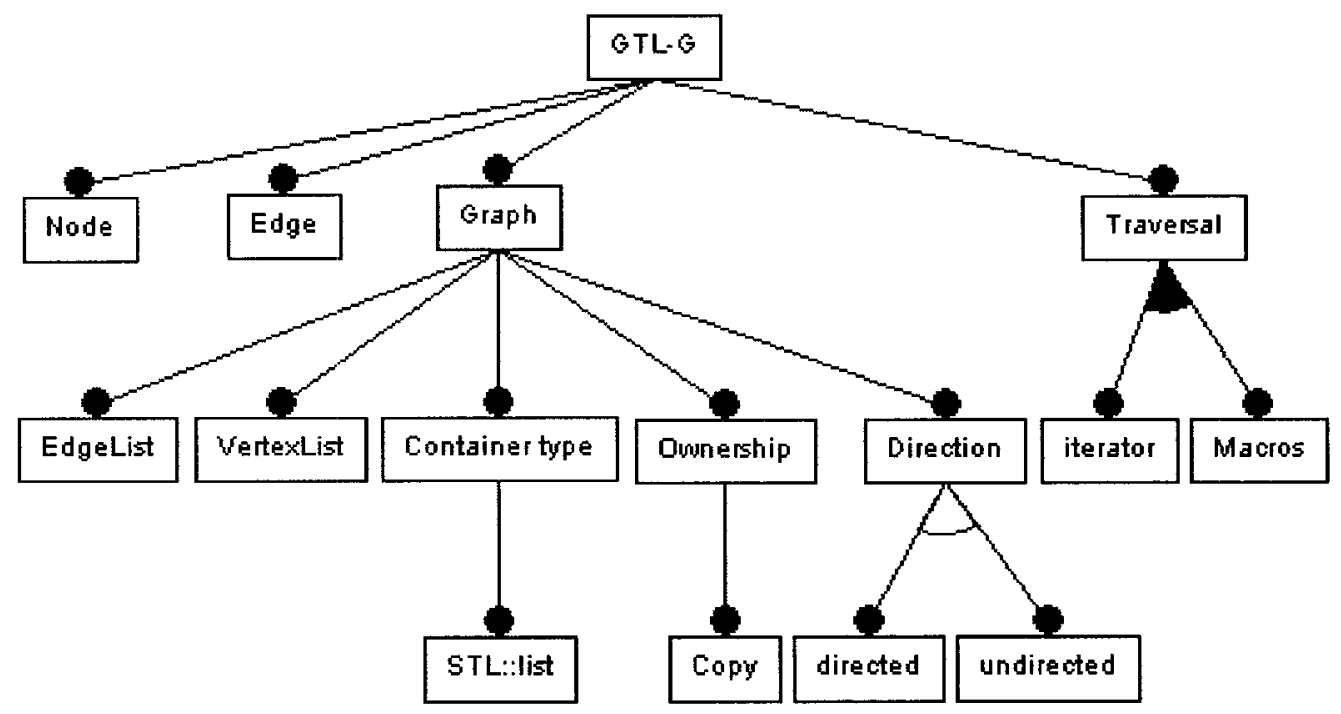

Figure 3-1 Feature diagram - GTL-G 
Although this diagram may appear self-explanatory, it rests on the assumption we make throughout this dissertation (following Bosch) that features are to be functional logical units [21]. In other words, contrary to some authors [40], no attempt is made here to directly address requirements (in particular non-functional ones) in our feature diagrams. Here is a brief description of each feature:

\section{Feature Name: Node}

Node is one of two basic items in graphs. A node in GTL-G has some information associated with it. It has an ID number, two lists used to store in-edges and out-edges, a pointer to the graph it belongs to and a list iterator to keep its position in the list of all nodes. The information is kept in class node_data. The node class itself provides an interface to access information, e.g. int outdeg() const which returns the number of outgoing edges of the node.

\section{Feature Name: Edge}

Edge is the other basic item in graphs. An edge in GTL-G has an ID number, two node lists to store source nodes and target nodes, an iterator list to keep positions in the adjacency lists of sources and targets, a pointer to the graph it belongs to and a list iterator to keep its position in the list of all edges. The information is stored in class edge_data. The edge class provides an interface to access information, e.g. node source() const which returns the source node of the edge. 


\section{Feature Name: Graph}

GTL-G uses an adjacency list to represent the graph. This feature has five sub-features (or "feature values"):

EdgeList: the interface for edge list.

VertexList: the interface for vertex list.

Container type: the container used to store graph items.

GTL-G uses STL::list to store edges and vertices.

Ownership: It has a value of Copy since the items stored in two lists are copies of vertices and edges.

Direction: A GTL-G graph can be either directed or undirected. It provides methods to change this property. So it has two variant values: directed and undirected.

\section{Feature Name: Traversal}

GTL-G uses iterator in STL::list to search a graph. It also has implemented macros, forall_nodes and forall_edges, to traverse a graph.

GTL-G has limited variability: users are given very few options. Everything is fixed except for the direction, which can be changed at runtime. In particular, a GTL-G graph has a fixed item type (i.e., a graph is forced to be homogeneous) and its node and edge classes are not changeable. Also, we repeat, the adjacency list is the only data structure it has. And, it uses only STL::list to support the adjacency list: users have no other choice. 
Simplicity is the dominant characteristic of GTL-G: no parameterization, little variability; everything is ready to use in GTL-G! This is the biggest virtue of GTL-G. There is no need of extra work from users. Because of low variability, there is little room for customization of representation or optimization through combinations of features. For example, performance suffers from having GTL-G keep much information in it, whether needed or not. In node_data, a list is used to keep out-edges no matter they are needed by users or not. For those who only care about functional requirements or require simplicity of use before anything else, GTL-G is a good choice. For those who care more about performance and flexibility, it may constitute a poor choice. Finally, we report that feature extraction was still arduous (due to complex coupling despite clean code style) but that the feature model is indeed useful (e.g., to quickly recall details after not having worked with the library for some time). 


\subsection{Graph Template Library from Russia (GTL-R)}

There are four categories of classes in GTL-R [5]:

1. Graph items. They are vertices (i.e., nodes) and edges. A graph is considered to store items, which originate in the application's data domain.

2. Data structures for storing graphs. For every data structure (i.e., every model used for graph representation) a set of the basic operations is implemented: adding and deleting of vertices and edges, iteration through graph items. Also, such structures can be stored in files (i.e., serialization is provided). The library allows the user to change the graph representation model, as well as using several models together.

3. Structures for representation of calculation results. Several special classes are developed for storing results of typical operations, with the possibility of using these classes as an input for other algorithms.

4. Abstract methods. A separate class is used to represent every abstract method, that is, every procedure that can be defined independently of representation! The following methods have already been implemented: depth first search, breadth first search, and the branch-and-bound algorithm. Everyone of these methods is meant to participate in the solution to a large amount of problems. The user is left with the task of specifying which sequence of such reusable methods must be followed to solve a specific for the problem.

We restrict our model to the first two of these four categories. This decision proceeds from two observations. First, algorithms (i.e., abstract methods) were dropped from our modeling task for they constitute "customers" of graphs rather than participating in the structure and/or behavior of graphs. Furthermore, as the 
dissertation of another member of our group [39] demonstrates, developing a unifying conceptual framework for a family of algorithms is a highly complex task on its own, one that lies beyond the scope of this dissertation (but is necessary)! From a software engineering viewpoint, we are following the widely used principle of separation of concerns. From a conceptual viewpoint, this separation is important. Let us elaborate. Graph algorithms can be viewed as the customers of graphs. By decoupling the customer from the provider, we greatly simplify variability by splitting it across the two domains. In other words, variability in graph representation is made to be independent (i.e., abstracted out) of variability in algorithms that use these graphs. From our viewpoint this is highly desirable for it maximizes the reusability of the algorithms across different graph representations. And it maximizes the possibility of testing an algorithm independently of a specific representation. Absence of such decoupling would likely lead to an explosion of feature combination rules that may not be necessary (e.g., tying specific algorithms to specific data structures ${ }^{11}$ ). The generation of tests constitutes a good heuristic for motivating this separation of concerns: test on the graphs proper can be developed regardless of the specific algorithm(s) that would used them. The possibility and desirability of testing graph algorithms and graphs independently of each other seems to vote in favor of the proposed separation.

Second, if algorithms are not addressed here, it follows that the structures used to represent the results of such algorithms can also be postponed to future research.

11 Tying an algorithm to a specific graph representation should be viewed as an optimization that follows the selection of a specific library. That is, only once variability has been modeled in each domain should we consider specific combinations of features across domains. 
Given we are limiting ourselves to graph items and graph representations, we could assume an easy ride through the library. But, alas, GTL-R documentation is only available in Russian in [5]. Thus, for the author, who doesn't know Russian, the only way to learn about GTL-R is to read its code.

GTL-R uses MFC (Microsoft Foundation Classes) containers to store graph items. This makes it quite different from the other graph libraries we considered. Here we use the edge class as an example of what we are faced with:

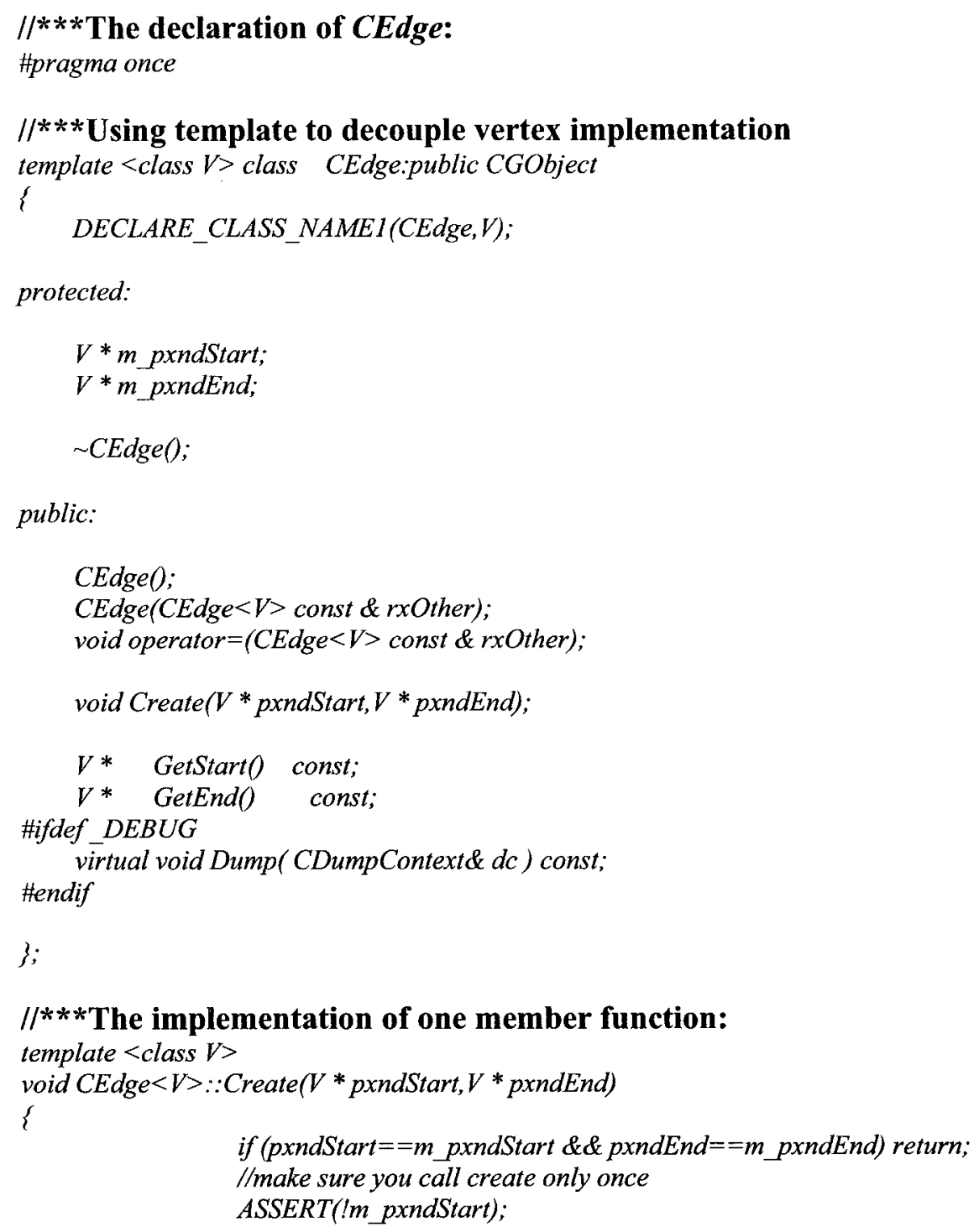




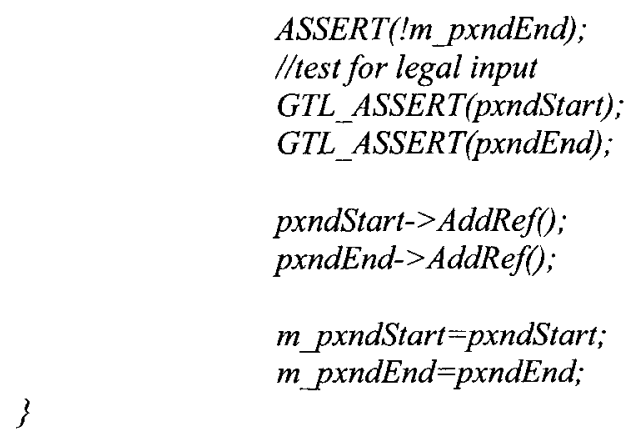

So far the code is simple and easy to understand.

$/ / * * *$ The other edge class $C W E d g e$ :

\#define EDGEPTRARRAY CTypedPtrArray $<$ CPtrArray, $E^{*}>/ 1^{* * *}$ Using MFC container

\#define EDGEPTRLIST CList $<E^{*}, E^{*} \&>/ /^{* * *} \mathbf{U}$ sing MFC container

$/ / * * *$ Taking $C E d g e<V>$ and $C W e i g h t F l a v o r$ as base classes

template <class $V>$ class $C W E$ dge:public $C E d g e<V>$, CWeightFlavor

\{

DECLARE_CLASS_NAMEI(CWEdge,V);

protected:

double $m_{-} d$ Weight;

public:

CWEdge $<\boldsymbol{V}>0$

$C W E$ dge $<V>$ (double dWeight)

$\{$ SetWeight $(0.0) ;\}$

$C W E$ dge $<V>$ (CWEdge $<V>$ const \& rxOther $)$

$\left\{r x O t h e r . m_{-} d W e i g h t=m_{-} d W e i g h t ;\right\}$

$\{$ SetWeight(dWeight $) ;\}$

\{operator $=($ rxOther $) ;\}$

void operator $=(C W E d g e$ const \& rxOther $)$

\{

$C E d g e<V>::$ operator $=(r \times O$ ther $)$;

\}

SetWeight(rxOther.GetWeight0);

\#ifdef_DEBUG

virtual void Dump (CDumpContext\& dc) const

\{

CEdge $<\boldsymbol{V}>\because: D u m p(d c)$;

\}

CWeightFlavor::Dump $(d c)$;

\#endif

virtual void Serialize( CArchive\& ar)

\{

CEdge $<V>::$ Serialize(ar);

if (ar.IsStoring()) ar $<<m$ dWeight;

子 else ar $>>m_{-} d$ Weight;

virtual void SetWeight(double $d$ Weight $) \quad\left\{m_{-} d W e i g h t=d W e i g h t ;\right\}$

virtual double GetWeight) const \{return $m$ dWeight;

virtual void Import(char * pBuffer,LPCSTR $x$ CurTemplate)

\{

//CGObject::Import(pBuffer,xCurTemplate); for (UINT $i=0 ; i<$ strlen $(x$ CurTemplate); $i++)$

\{

if $(x$ CurTemplate $[i]==$ CWeightFlavor::GetTemplate ()$)$

\{ 


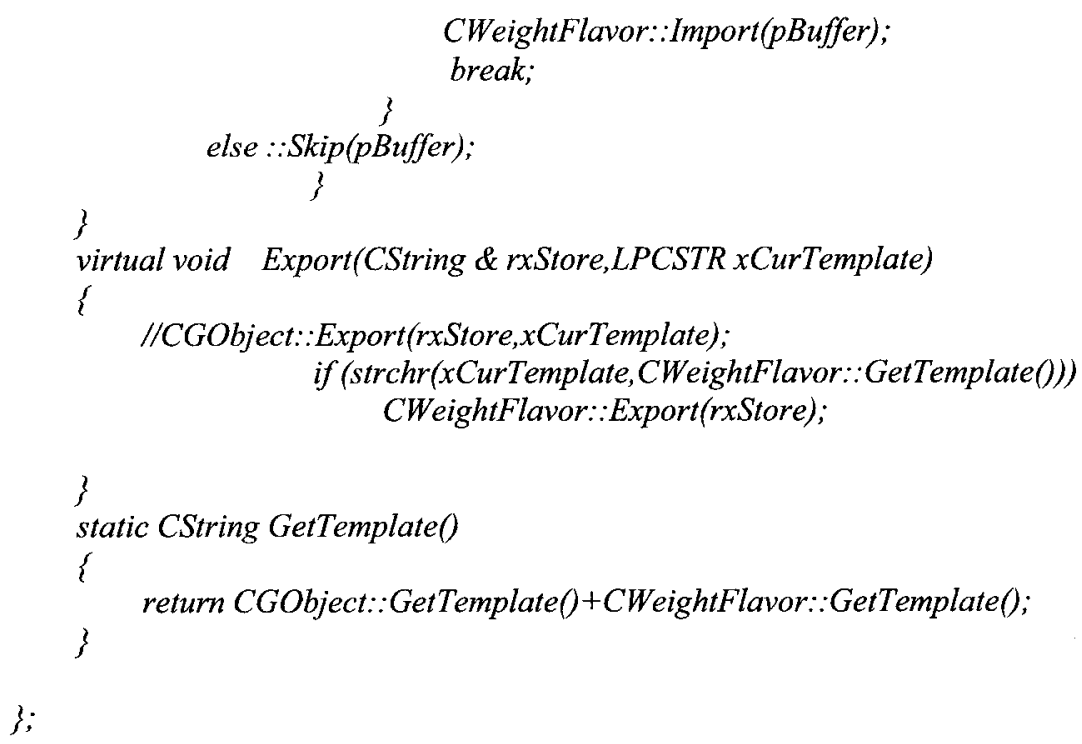

Templates and inheritance make the code very simple to decipher. But we still need to know all variants of edge classes in order to understand the edge class itself. We have found that the design decisions behind the edge class are quite clever. Thus, it is a pity that designers haven't documented them is an explicit way. We had no idea about them until we went through almost all classes in the library! In fact, a first comer to the code will unavoidably ask himself why there are so many vertex and edge classes.

After reading over the code (albeit several times), we come up with the following feature diagrams for GTL-R: 


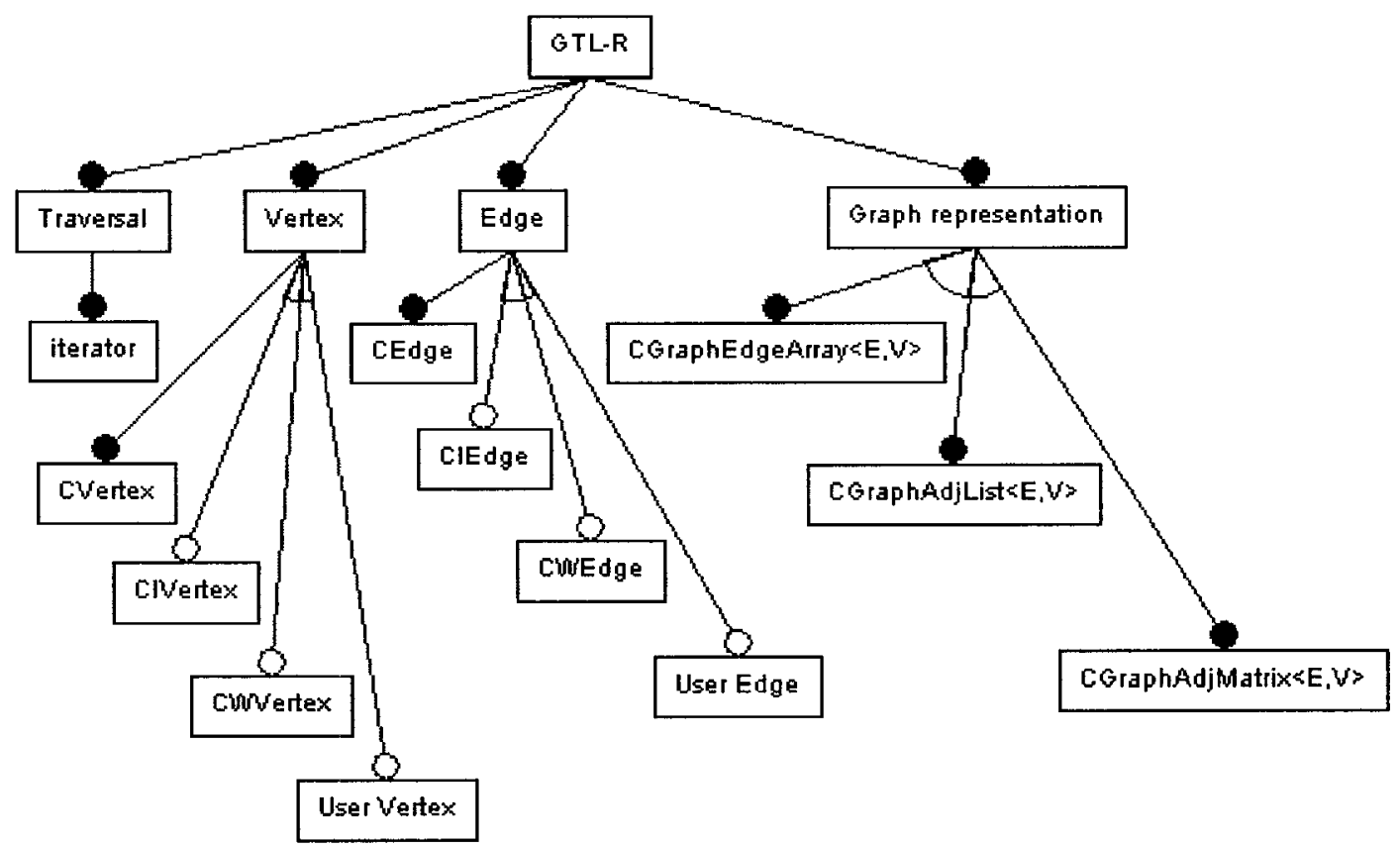

Figure 3-2.a Feature diagram - GTL-R

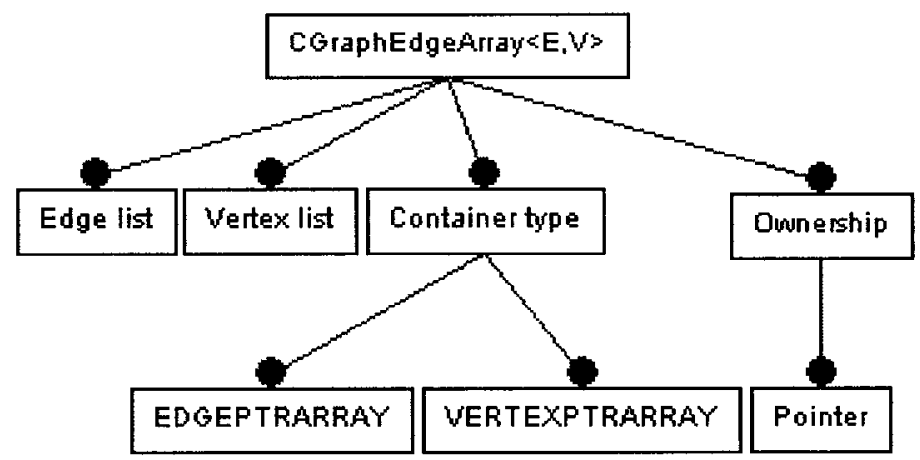

Figure 3-2.b Sub-feature CGraphEdgeArray 


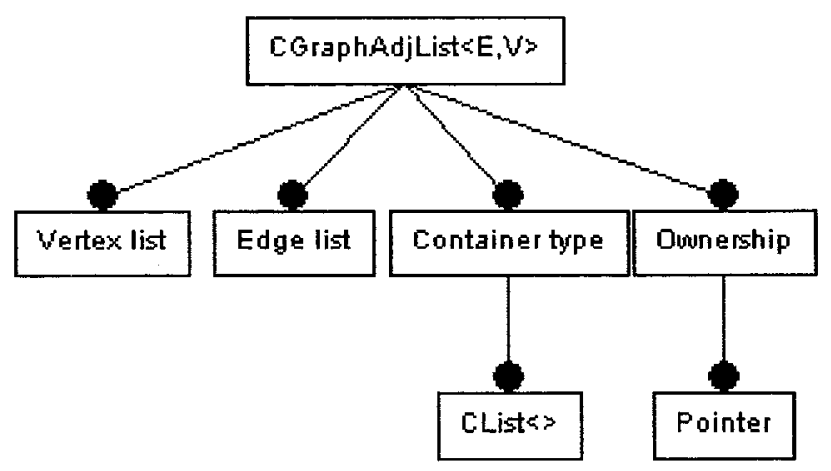

Figure 3-2.c Sub-feature CGraphAdjList

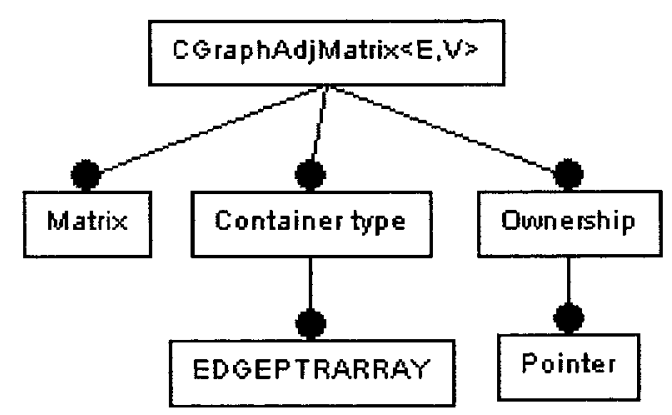

Figure 3-2.d Sub-feature CGraphAjdMatrix

We document the proposed features in the following pages.

\section{Feature Name: Vertex}

The vertex type in GTL-R is selectable. The library provides four options for it. Therefore we have four feature values under this feature:

CVertex: Base class of other vertex classes.

CIVertex: A vertex type, which can be numbered (or indexed).

CWVertex: A vertex type, which can be weighted.

UserVertex: User-defined vertex type.

CVertex is mandatory since it is the base class. The other three are optional: they simply may be used to substitute for the base. In 
other words, we are trying to express inheritance in our diagram by using different feature types... We acknowledge that such modeling is a bit clumsy...

Feature Name: Edge $<\mathbf{V}>$

In GTL-R, all edge classes take Vertex as a template parameter. We are trying to reflect this by using Edge $<\mathrm{V}>$ as the feature name.

This feature itself is selectable too. It has four values.

CEdge: Base class of other edge classes.

CIEdge: An edge type, which can be numbered (or indexed).

CWEdge: An edge type, which can be weighted.

UserEdge: User-defined edge type.

CEdge is a mandatory value. The other three are optional. Again, through this strategy, we are trying to capture the inheritance relationship in the same way we did for Vertex.

\section{Feature Name: Graph Representation}

GTL-R implements three graph representations. They all take Vertex and Edge as parameters. This feature thus has three alternative values:

\section{CGraphEdgeArray $<E, V>$ :}

Using Edge Array to represent graphs. It uses lists to store 
vertices and edges. The container type is a parameterized pointer array (CTypedPtrArray) in MFC. Items stored in the containers are pointers.

Arrays in MFC are similar to C arrays, but can dynamically shrink and grow as necessary [34]. The access time for an indexed element is constant and is independent of the array size [34]. The definition of CTypedPtrArray is like this: template < class BASE_CLASS, class TYPE > class CTypedPtrArray : public BASE_CLASS $B A S E \_C L A S S$ must be an array class (CObArray or CPtrArray) [34]. Here GTL-R uses CPtrArray, which supports arrays of void pointers. TYPE is the type of the elements stored in the base-class array [34].

\section{CGraphAdjList $<$ E, V>:}

Using Adjacency List to represent graphs. In this representation, a list is used to store vertices. Each vertex stored in the list has two associated lists to store in-edges and out-edges. This graph representation uses CList class in MFC (template < class TYPE, class ARG_TYPE $>$ class CList : public CObject) to store elements. Thus the container type in the feature diagram is CList $<>$. The CList class supports ordered lists of non-unique objects, accessible sequentially or by value [34]. CList lists behave 
like double-linked lists [34].

Elements stored in Clist are pointers to graph items. Thus the feature Ownership has a value of pointer.

\section{CGraphAdjMatrix $<E$, V $>$ :}

Using Adjacency Matrix to represent graphs. In this representation, an array class in MFC (CTypedPtrArray) is used to store edges. The value of container type is EDGEPTRARRAY, which is defined as a CTypedPtrArray.

The array stores pointers to edges. So the Ownership has a value of pointer.

\section{Feature Name: Traversal}

GTL-R uses the relevant iterator in MFC to search the graph. Thus this feature has a feature value: iterator.

Both MFC and STL provide a facility to iterate over all of the objects within a container. However, the approach taken is different. Within the MFC, the iteration mechanism is dependent upon the container, while in the STL there is a common iteration mechanism so that an algorithm can operate on each element of a container without knowledge of the container's type. [35]. But such a 
distinction is not relevant to the user of a graph. And thus we treat the two approaches as a single feature iterator provided by the container.

Inheritance and templates contribute to the efficiency of GTL-R, although they make the library more complicated to use than GTL-G. The use of inheritance makes it possible to keep the least information needed and provides a unified interface. The use of templates provides an elegant way to combine information without creating spurious classes. For example, for a breadth-first-search algorithm, the color of each vertex needs to be kept. Conversely, edges do not need to maintain color. So we can select CIVertex as the vertex class, and base edge class $C E d g e$ as edge class. Because $C E d g e$ takes $C I V e r t e x$ as a template parameter, we obtain exactly what we want without any attributes propagating incorrectly to other classes.

GRL-R has great flexibility, in particular because it has implemented all three popular graph representations. As mentioned before, these three representations have different characteristics; especially, they have different time and space complexities. For those who need to support multiple graph representations or need to change from one representation to another, GTL-R is a good choice.

Finally, understanding complexity is not trivial in this library. The difficulty comes from the fact that the model of reuse involves combining classes through inheritance and templates. Such a task requires a deep comprehension of inter-class relationships, as well as considerable experience at grasping the 
consequences (especially with respect to performance) of some combinations of classes over others.

\subsection{Boost Graph Library (BGL):}

BGL is claimed to be generic, in the same sense as the Standard Template Library (STL) [6]. There are three ways in which BGL is generic [6]:

- Algorithm/Data-Structure Interoperability (the same as in STL) In STL, each algorithm is written in a data-structure neutral way, allowing a single template function to operate on many different classes of containers. The concept of an iterator is the key ingredient in this decoupling of algorithms and data-structures. The impact of this technique is a reduction in the STL's code size from $O\left(M^{*} N\right)$ to $O(M+N)$, where $M$ is the number of algorithms and $\mathrm{N}$ is the number of containers. [6] Like the STL, the graph algorithms of BGL are written using an interface that abstracts away the details of a particular graph data-structure. And BGL also uses iterators to define the interface for data-structure traversal. [6]

- Extension through Visitors (STL uses Function Objects)

The second way STL is generic comes from the fact that its algorithms and containers are extensible: The user can adapt and customize the STL through the use of function objects. [6]

The graph algorithms of BGL are also extensible. In order to enable this, BGL introduces the notion of a visitor [13] which is realized as a function object with multiple methods. In graph algorithms there are often several key "event points" at which it is useful to insert user-defined operations. 
The visitor object has a different method that is invoked at each event point. The particular event points and corresponding visitor methods depend on the particular algorithm. They often include methods like start_vertex(), discover_vertex(), examine_edge(), tree_edge() and finish_vertex(). [6]

- Vertex and Edge Property Multi-Parameterization (STL uses Element Type Parameterization)

The third way that STL is generic is that its containers are parameterized with respect to the element type. [6]

The third way that BGL is generic is analogous to the parameterization of the element-type in STL containers. Values must be associated with both the vertices and the edges of the graph: such an associated value is also called a property. In addition, it will often be necessary to associate multiple properties with each vertex and edge, which is multi-parameterization is supported. [6]

BGL currently provides two graph classes that implement a generalized adjacency list: adjacency_list and adjacency_matrix. The adjacency_list class is the general purpose class. It is highly parameterized so that it can be optimized for different situations. For example, a graph is directed or undirected, allows or disallows parallel edges, provides or not efficient access to just the out-edges, or also to the in-edges, supports fast vertex insertion and removal at the cost of extra space overhead, etc. [6]

The main link between the abstract mathematical nature of graphs and the concrete problems they are used to solve is the properties that are attached to the vertices 
and edges of a graph: e.g., distance, capacity, weight, color, etc [6]. BGL favors the abstraction of properties out of graph and graph items. There are two kinds of properties in BGL: interior and exterior. Interior properties are stored "inside" the graph object in some way, and the lifetime of the property value objects is the same as that of the graph object. Exterior properties are stored "outside" of the graph object and the lifetime of the property value objects is independent of the graph. This is useful for properties that are only needed temporarily, perhaps for the duration of a particular algorithm, such as the color property used in breadth_first_search (). [6] The property map interface in BGL provides a generic method for accessing properties from graphs [6]. It defines several functions. Here is an example from [6]:

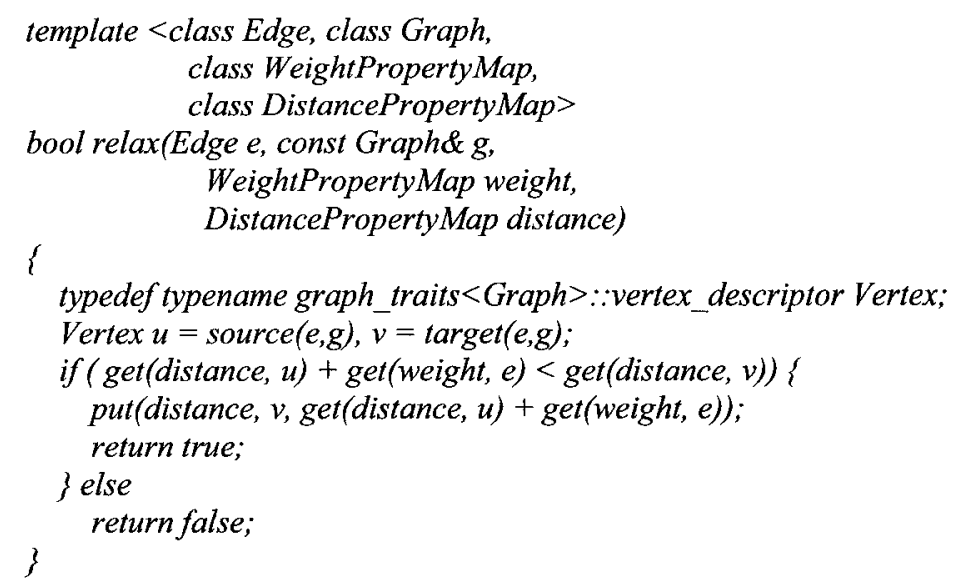

Although the documentation is very detailed (e.g., even some design decisions are documented), most of the content of documentation is about implementation details. In order to get a conceptual idea we still need to go through the code. And again, though the code in each single class may be simple, the relationships between classes are very complicated. It took us a long time to go understand the design of BGL. Let us take a look at some code in it. Here is the base edge class:

$/ / * * * * *$ Base class

template <typename Directed, typename Vertex> 
struct edge_base

\{

inline edge base() \{\}

inline edge base(Vertex $s$, Vertex d)

$: m_{-} \operatorname{source}(s), m_{-}$target $(d)\{\}$

Vertex $m$ source;

\} ;

Vertex $m$ target;

\section{$/ / * * * * *$ Implementation}

template <typename Directed, typename Vertex>

class edge_desc_impl : public edge_base $<$ Directed,Vertex $>$ \{

typedef edge_desc_impl self;

typedef edge base $<$ Directed,Vertex $>$ Base;

public:

typedef void

property_type;

inline edge_desc_implo:m_eproperty $(0)\{\}$

inline edge_desc_impl(Vertex $s$, Vertex d, const property_type* eplug)

: Base $(s, \bar{d}), m_{-}$eproperty(const_cast<property_type ${ }^{*}>($ eplug $\left.)\right)\{\}$

property_type* get_property0 \{ return m_eproperty; $\}$

const property_type* get_property) 0 const $\{$ return $m$ eproperty; $\}$

$1 /$ protected:

\}

property_type* $m_{-}$eproperty;

The edge class can take any Vertex type by using a template parameter. Let us see how Vertex and Edge are defined in Adjacency_list:

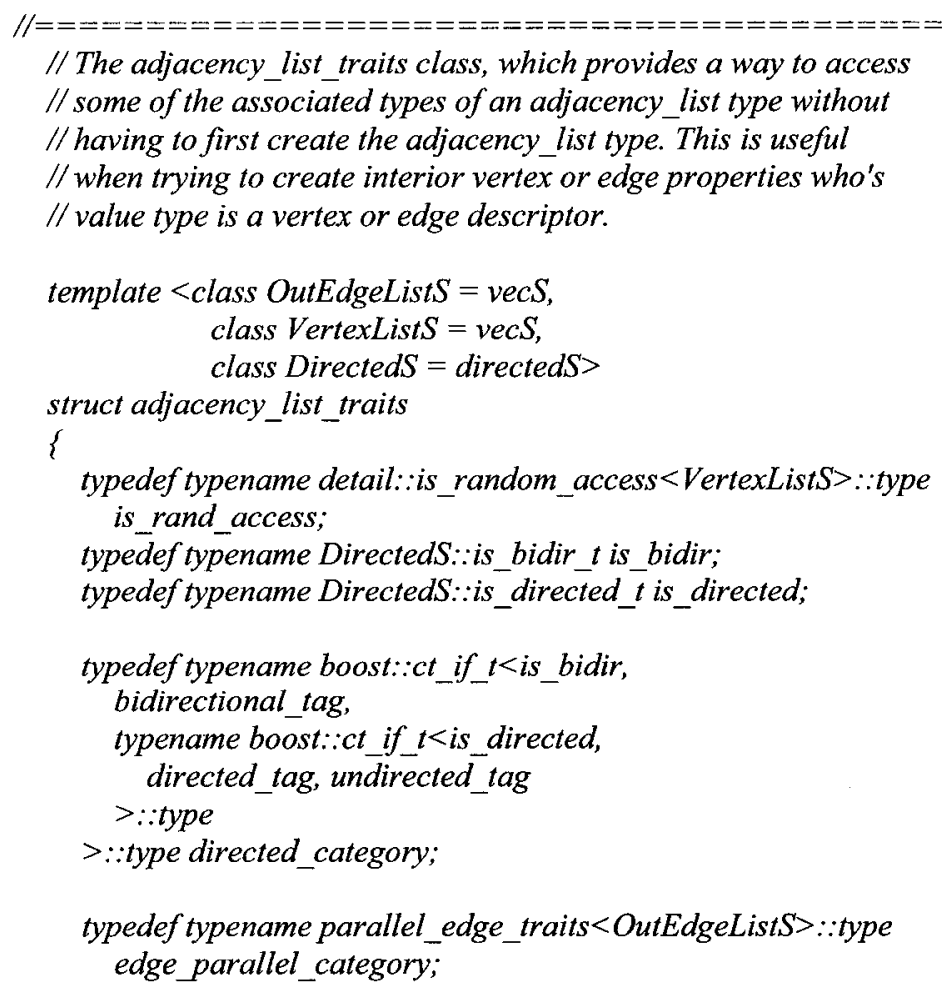




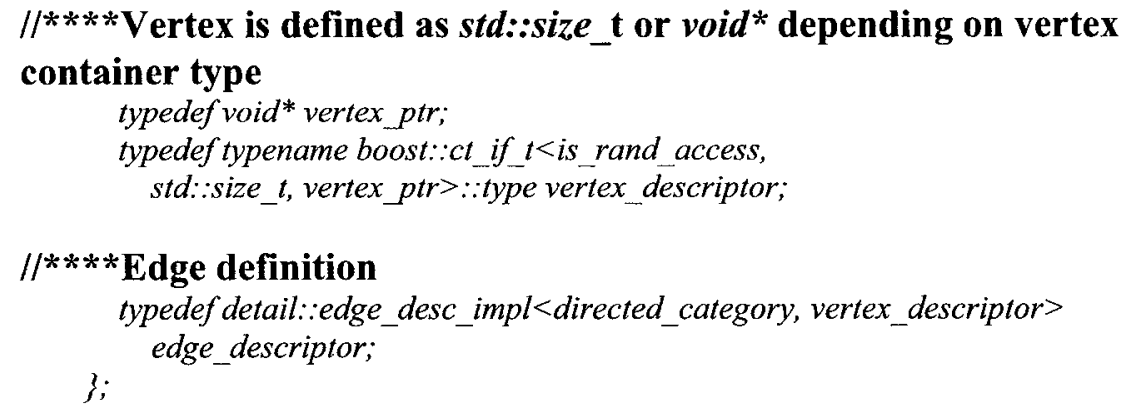

Clearly, the use of templates (if not of meta-programming techniques [11]) may make the code very efficient, but at the expense of understandability. In fact, we notice that some design decisions pervade a multitude of classes. Such as traits classes in BGL(graph_traits, adjacency_list_traits and property_map), associated types can be accessed without instantiation by using templates.

Here is our proposed feature diagram for BGL: 


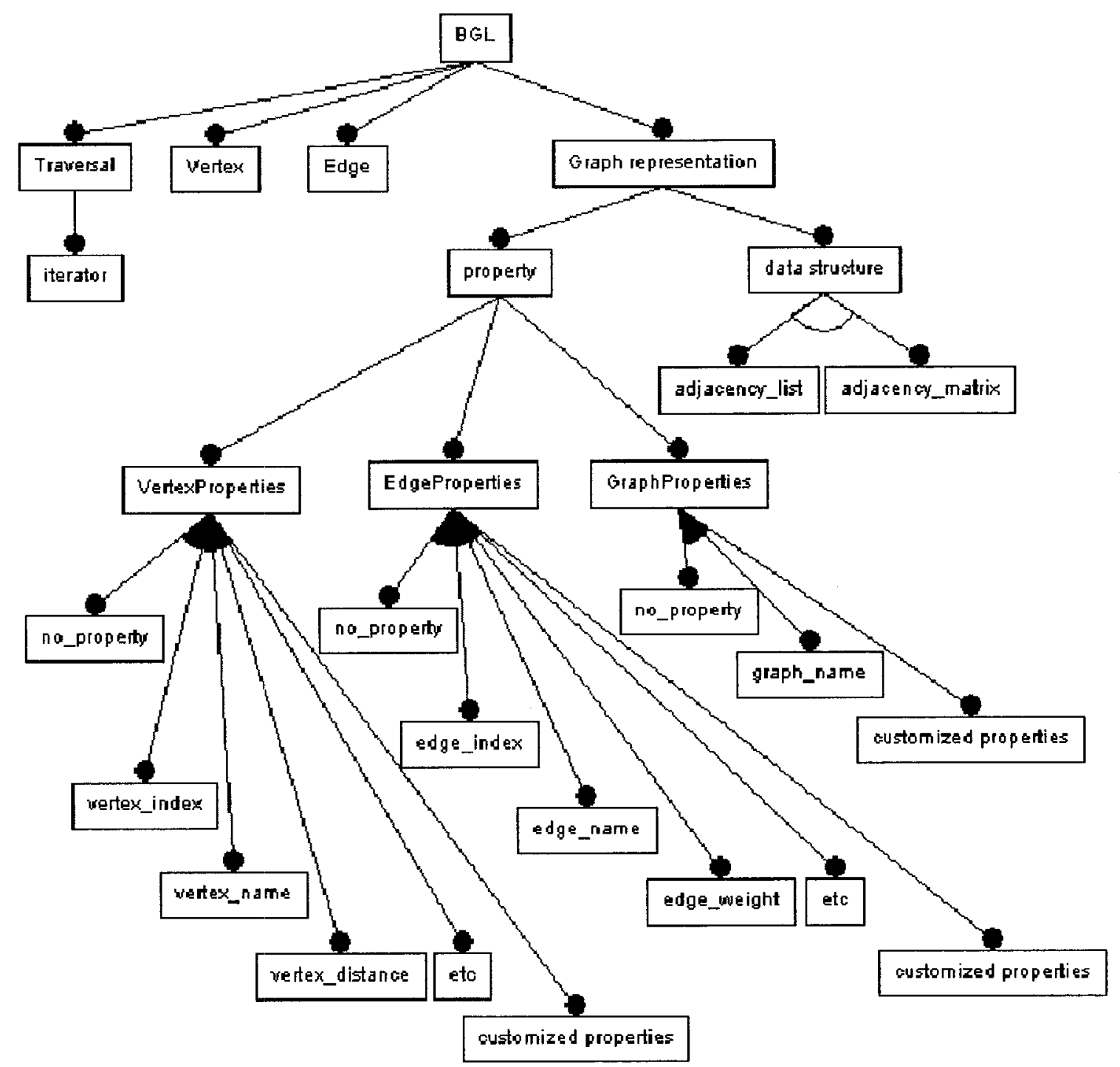

Figure 3-3.a Feature diagram - BGL 


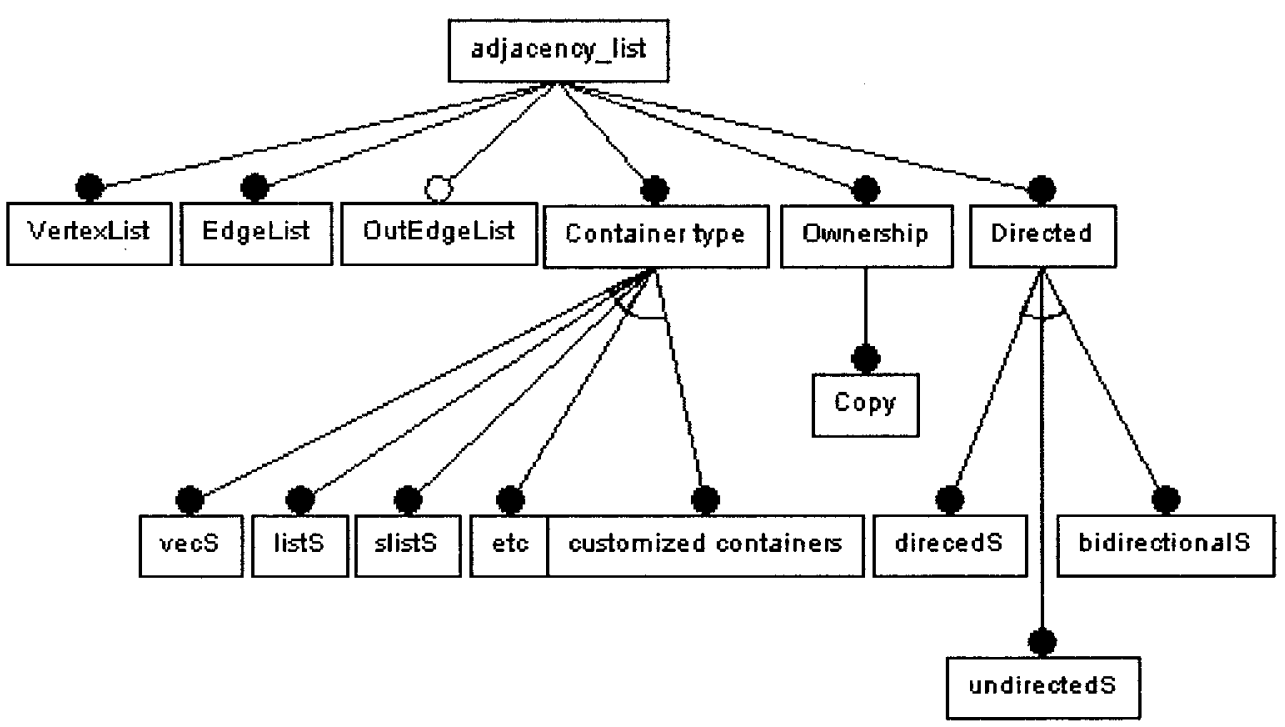

Figure 3-3.b Sub-feature - adjacency_list in BGL

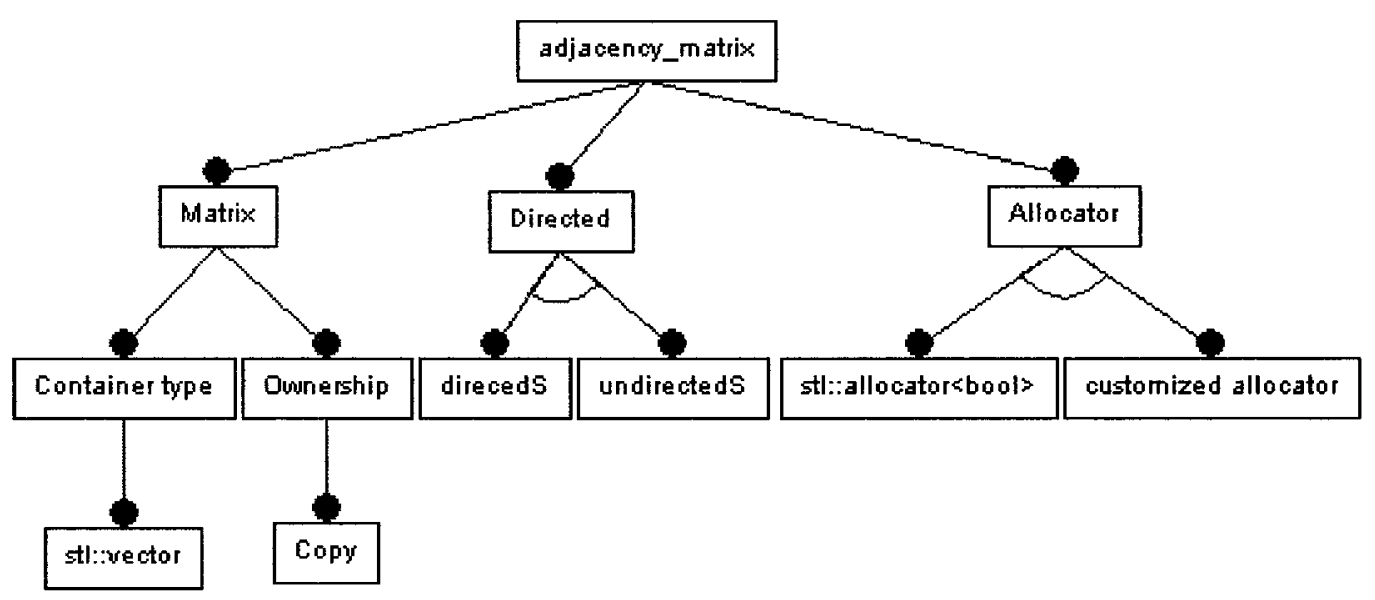

Figure 3-3.c Sub-feature - adjacency_matrix in BGL

Feature Name: Vertex

The vertex in BGL may be defined as std::size_t or void *. No information is associated with it.

Feature Name: Edge

The edge in BGL is very simple. It only keeps its source vertex and 
target vertex as copies. No other information is associated with it.

Feature Name: Graph Representation

There are two sub-features under this feature: properties and data structure. BGL offers two data structures:adjacency_list or adjacency_matrix.

\section{adjacency_list:}

It uses three containers to store graph items. VertexList is the container interface used to represent the vertex-list of the graph.

EdgeList is the container interface used to represent the edge-list for each of the vertices. OutEdgeList is an optional feature value. It is the container interface used to represent the out-edge-list for each of the vertices in a bidirectional graph.

The actual container type is selectable through a container selector. It can be vector, list and map in STL or user-defined types.

Graph items stored in containers are copies. Thus, the value of Ownership is copy.

The feature Directed has three alternative values: directed selector, undirected selector and bidirectional selector. Thus a graph represented using an adjacency_list can be directed, undirected or bidirectional. In BGL, a bidirectional graph is a directed graph with bidirectional edge access (access to both out-edges and in-edges). A bidirectional graph is a directed graph in which for each edge $(u$, $v) \in G,(v, u) \in G$. Bidirectional graphs can be treated as undirected 
graphs given $(u, v)$ and $(v, u)$ have the same properties. BGL has implemented this concept in case it is required to use an extra list to store out-edges.

adjacency_matrix:

It uses a matrix to store edges. In this data structure, the container type is fixed by using stl::vector, as opposed to user-selectable containers for adjacency_list. More specifically, BGL uses a one dimensional container to represent a matrix. When the graph is undirected, half of the storage space will be saved [2].

Edges stored in the matrix are copies. So the Ownership has a value of Copy.

The feature Allocator is used to allocate memory for the matrix. The default value for this feature is stl::allocator $<$ bool $>$. But it can be user-defined.

The direction for this kind of graph is directed or undirected. No bidirectional value is offered for this feature.

The graph representation feature also has a sub-feature properties. As mentioned before, BGL abstracts properties out of the implementation of graphs and graph items. All properties are selectable via a template parameter list.

Noticing that the two possible data structures of a graph representation have the same property selectors, we separated properties from data structures. The properties feature has itself three 
sub-features: VertexProperties, EdgeProperties, and

GraphProperties.

\section{VertexProperties:}

One or more from no_property, vertex_name, vertex_index, vertex_distance and customized properties (which is an open door for user-defined properties). The default vertex property in BGL is no_property.

\section{EdgeProperties:}

One or more from no_property, edge_name, edge_index, vertex_weight and customized properties. The default edge property in BGL is no_property.

\section{GraphProperties:}

One or more from no_property, graph_name and customized properties. The default graph property in BGL is no_property.

\section{Feature Name: Traversal}

BGL has defined its own iterators based on container iterators. In our feature diagram, we classify this kind of iterator simply as iterator. The iterator may come from STL, MFC, from a combination of both, or from the graph library itself. This versatility explains why we simply used iterator as the feature 
under traversal, understanding feature iterator itself could have a multitude of possible feature values.

From this model, we can see the feature model can abstract complex design decisions into a simple and easy to understand model. There are abundant design decisions inside BGL. It will take much time to understand the library. Its documentation emphasizes the use of the library, not these decisions. Thus, such decisions are spread all over the code. In our opinion, our model is an easy way to get an idea of how everything works in the BGL.

BGL has excellent flexibility: Users can select graph representations, container type and properties. Different graph representations have different time and space complexities which we mentioned before. Different container types also provide different time and space complexity to meet user requirements. Users can also define their own container types and properties to meet their specific requirements. From this standpoint, BGL can be viewed as a framework.

High performance is another characteristic of BGL. Most aspects of a graph are decided at compile time through techniques similar to meta-programming [11]. This makes its code size is small while speed is fast. All information about graphs and graph items are stored separately in property-maps. This information can be selected by demand.

Complexity of use seems to be, as usual, the price to pay in order to have good performance and flexibility. In other words, BGL is not simple and easy to use: 
There are lots of ideas to master before one can use it. And, the onus placed on the user to produce code (as opposed to simply reuse it) is high: deep understanding of class inter-relationships and of advanced template usage techniques is required!

\subsection{LEDA}

The main feature of LEDA is its ease of use [7]. It allows us to write programs for graph problems in a form close to the typical textbook [2] presentation [4]. Put simply the design of LEDA claims "Algorithm + LEDA = Program" [4]. For example, consider Dijkstra's algorithm in [2]:

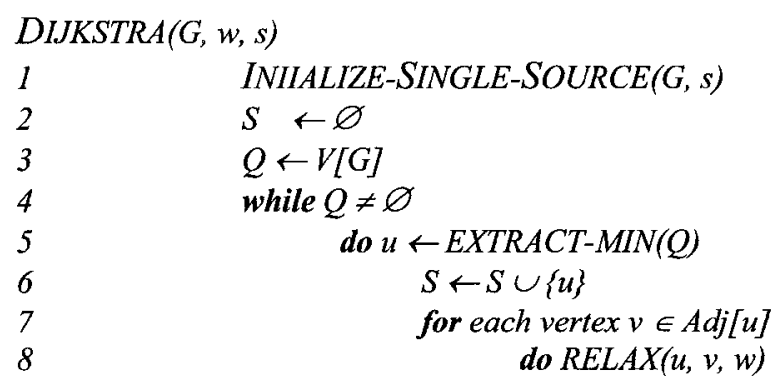

Let us study an example from [4] to illustrate how to use LEDA to implement this algorithm:

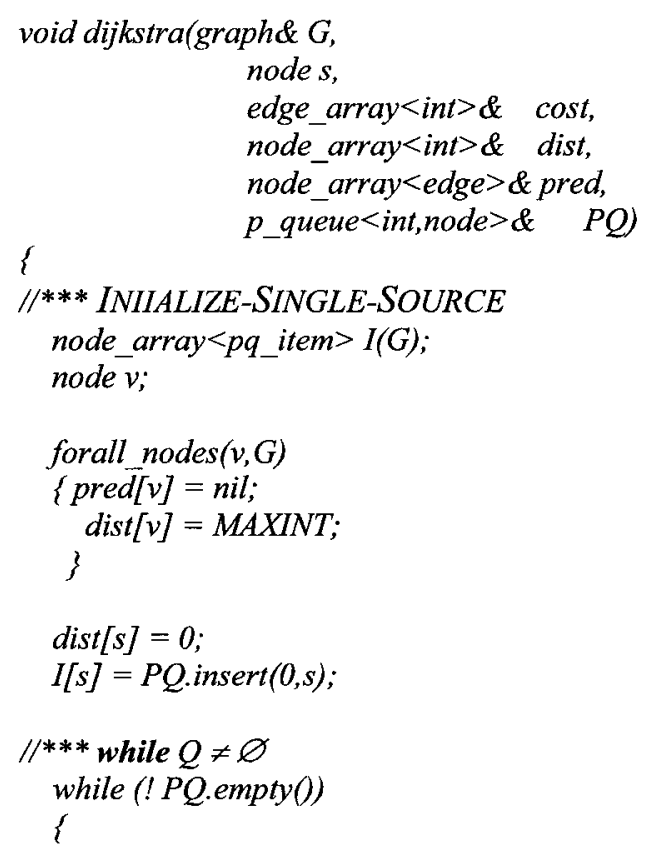




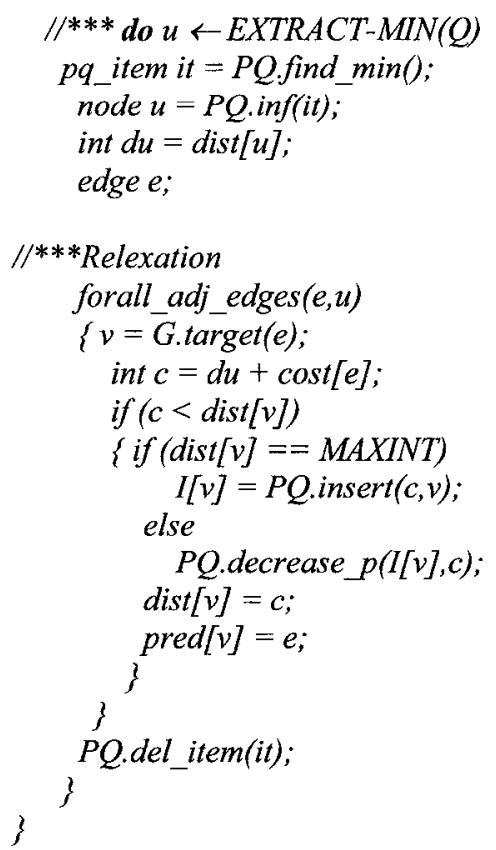

In our opinion, this example does show how close LEDA code is to the algorithm from the textbook [2].

We still need to go through the code in order to get a conceptualization of the design of LEDA. Its detailed documentation is available in [7]. There is also a tutorial available in [7]. But both are all about the use of the library, not how it was designed! High level design decisions are hidden deep in the code. Here is the sample code of edge class (inside graph_object0.h) in LEDA:

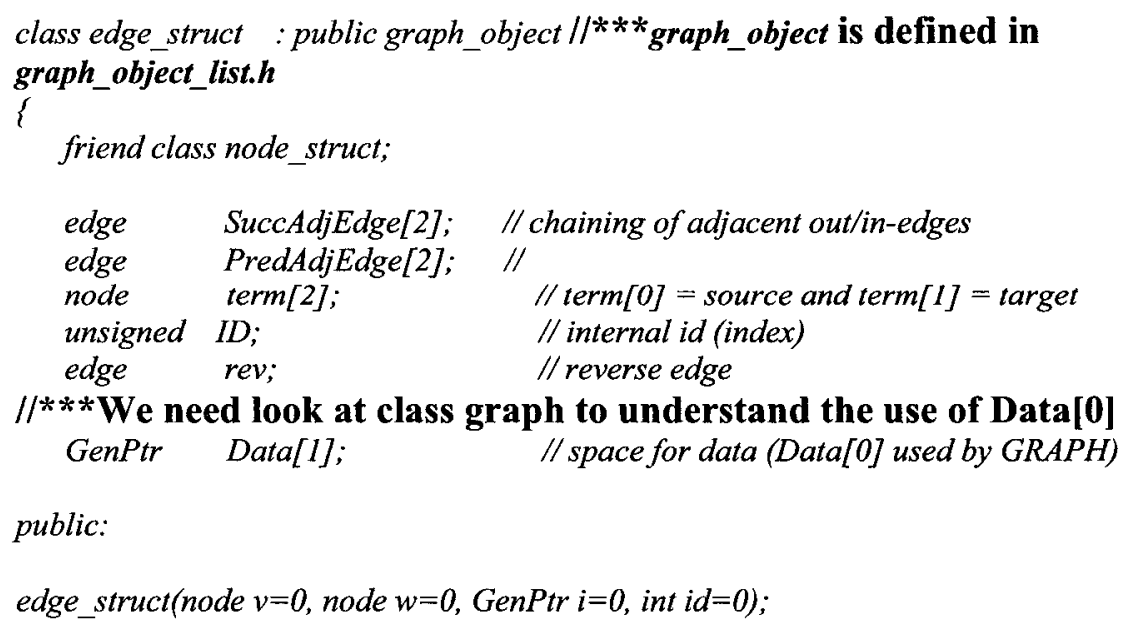




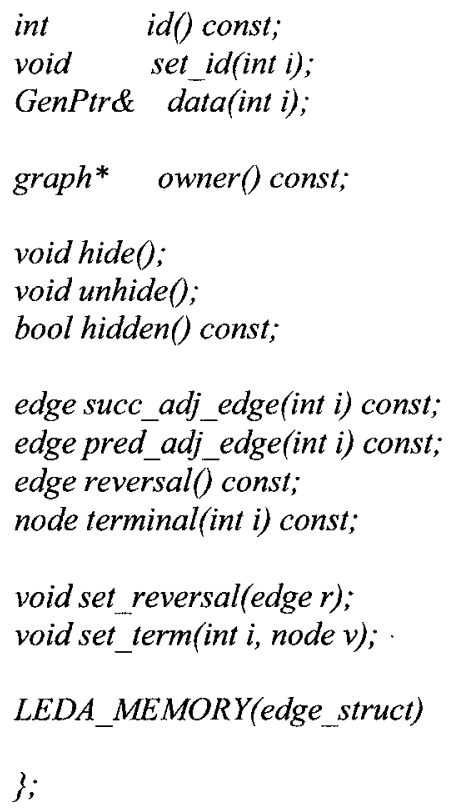

The coding style in LEDA is simple and plain. But this is, we repeat, a false impression: understanding requires a fair amount of work, especially, in regards to the use of edges.

Here is the feature model we propose for LEDA after going through the code.

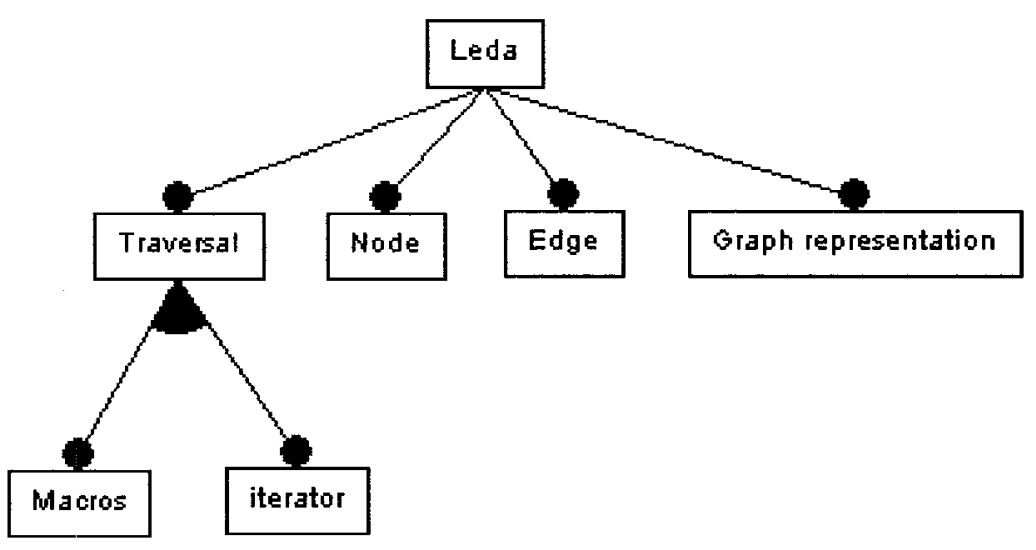

Figure 3-4.a Feature diagram - LEDA 


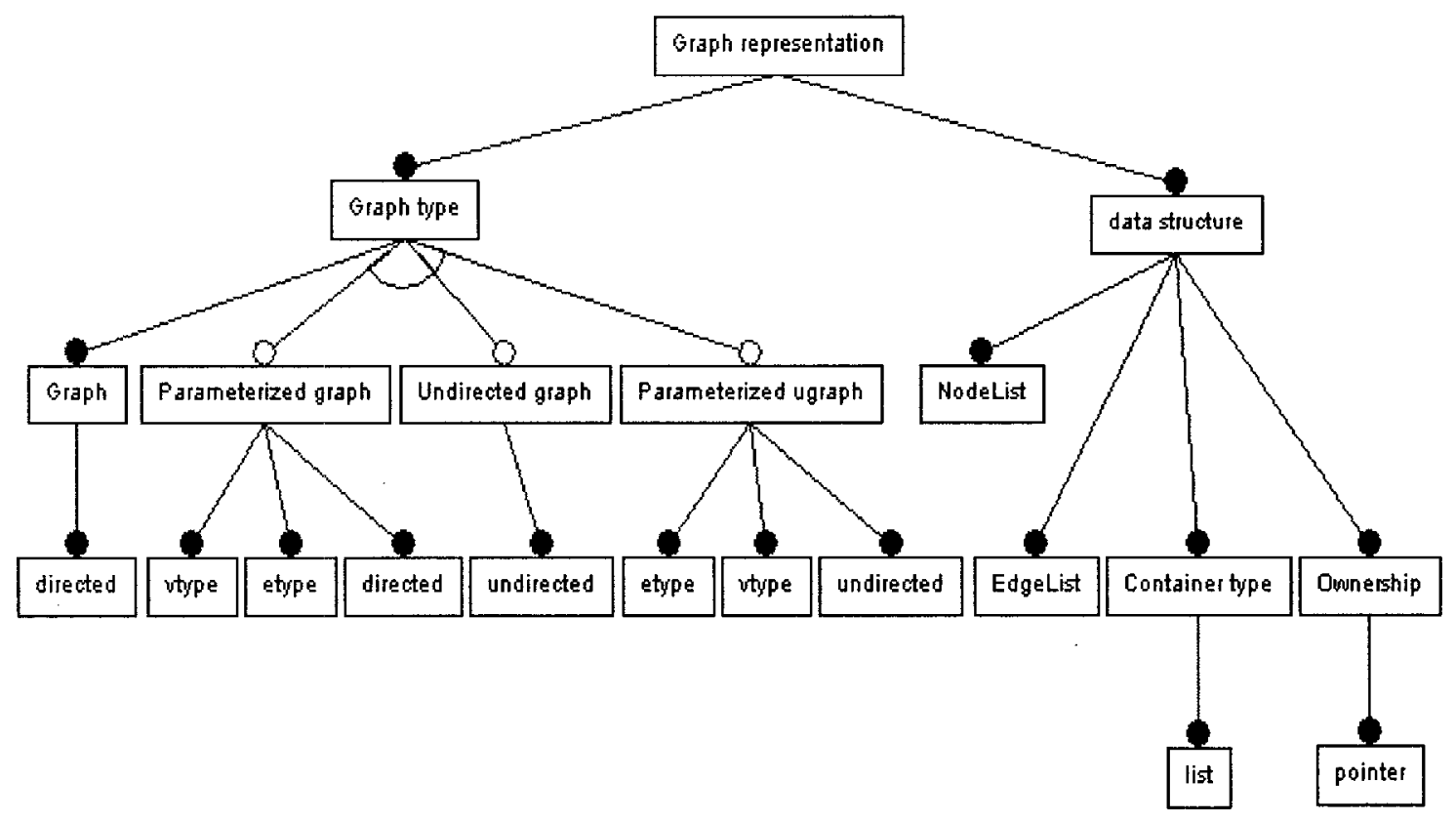

Figure 3-4.b Sub-feature - Graph representation in LEDA

Feature Name: Node

A node in LEDA has a lot of information attached to it: Nodes are stored in a node list (a double linked list). Therefore a node has two pointers to its preceding and succeeding nodes. It has an ID number as well. It has two arrays, each of two edge pointers to get access to the head and the tail of both the out-edge list and in-edge list separately. It also keeps its out degree and in degree. And it keeps a pointer to the graph it belongs to and a "general" pointer to keep extra data, such as the distance of the node. This data can also be user defined. The data is accessible through a map interface defined inside graph_map.h.

Feature Name: Edge

An edge in LEDA has much information associated with it. Like 
Node, Edge has two pointers to its preceding and succeeding edges. Edges themselves form an edge list (a double linked list). It has an array of two edge pointers to its predecessor in the adjacent in-edge list and out-edge list, an array of two edge pointers to its successor in the adjacent in-edge list and out-edge list. It also has two pointers to its source node and target node. It has an ID number. It keeps its reverse edge. It has a space to keep extra data, e.g. the weight of the edge. This data can also be user defined. The data is accessible through a map interface defined inside graph_map.h.

\section{Feature Name: Graph Representation}

LEDA has four graph types. They all use adjacency list as their data structure.

\section{Data structure:}

LEDA has only implemented adjacency_list to represent graphs. It uses two lists to store graph items. NodeList is the list of all nodes. EdgeList is the list of all edges.

LEDA provides its own container to store graph items, which is graph_object_list $<>$ defined inside graph_objects $0 . h$.

Graph items are stored inside list by pointers. Thus the value of Ownership is pointer.

\section{Graph type:}

There four kinds of graph classes although they all use 
adjacency_list as their data structure. We thus abstracted the data structure to a higher level of our model to decrease redundancy. The feature Graph type has four alternative sub-features:

\section{Graph:}

This feature is a mandatory sub-feature of Graph type since it is the base class of other three graph types. This sub-feature has only one value left: its graph type is necessarily directed.

\section{Parameterized graph:}

This type of graph is a subclass of graph. It is also directed. The difference between this type and the last one is that this type can take vtype and etype as template parameters. vtype and etype can be user defined types (for vertices and edges respectively). They are kept inside node class and edge class as node_data and edge_data.

\section{Undirected Graph:}

This type is a subclass of graph: it is simply undirected.

\section{Parameterized ugraph:}


This type is a subclass of Parameterized graph and is undirected. It is also parameterized. In this graph type data stored in nodes and edges are defined according to template parameters.

\section{Feature Name: Traversal}

LEDA has defined its own iterator and Macros (forall ()$)$ to traverse a graph.

As previously mentioned, ease of use is the main characteristic of LEDA. In particular, few classes need to be defined and templates are limited to very simple usages. Class inter-relationships are quite minimal and this also contributes to the overall simplicity.

But LEDA has limited flexibility. Adjacency_list is the only graph representation, we insist. And the library provides its own container and iterator: no customization is possible. The only thing flexible is the data type inside the node class and the edge class. Templates are limited to this simplistic use...

Finally, while we have not measure systematically performance for any of the libraries, we suspect performance using LEDA may be somewhat poor. This directly results from the fact that the node and edge classes have a lot of information 'hardwired' in them, be it needed for a certain application or not. This is worth emphasizing. It is often the case in software engineering that flexibility hinders performance. Conversely, lack of flexibility often suggests the use of 
optimizations to boost performance. But, in the case of LEDA, performance is NOT the main requirement, usability is. And these two requirements are in a trade-off relationship

\subsection{Library comparison}

Comparison is easier once (and only once, in our opinion) we have a feature model for each library. Why are there many graph libraries? What is the purpose of each library? What are advantages and disadvantages for each library? The point to be grasped is that such questions will not be answered by studying the documentation of each library. The design decisions are in fact almost always hiding behind the documentation and the code. In essence, the design of each library has to be extracted from the code. Following the work of Czarnecki and Eisenecker on libraries [11], our work also suggests that feature models are a useful tool to capture such design and their underlying decisions. These models act as blue prints for buildings: we don't have to 'traverse' two buildings to compare them if we have blue prints of them.

Now let us compare these four graph libraries using our proposed feature models.

Table 3-1 library comparison

\begin{tabular}{|c|c|c|c|c|}
\hline GTL-G & Vertex & Edge & Graph rep. & Traversal \\
\hline GTL-R & $\begin{array}{c}\text { Information by } \\
\text { demand }\end{array}$ & $\begin{array}{c}\text { Info. by } \\
\text { demand }\end{array}$ & $\begin{array}{c}\text { Adjacency_list } \\
\text { Adjacency_matrix } \\
\text { Edge_array }\end{array}$ & Iterator \\
\hline BGL & $\begin{array}{c}\text { No information } \\
\text { associated }\end{array}$ & $\begin{array}{c}\text { No } \\
\text { information } \\
\text { associated }\end{array}$ & $\begin{array}{c}\text { Adjacency_list } \\
\text { Adjacency_matrix }\end{array}$ & Iterator \\
\hline LEDA & Full size & Full size & Adjacency_list & $\begin{array}{c}\text { Iterator } \\
\text { Macro }\end{array}$ \\
\hline
\end{tabular}


Table 3-1 is not intended to represent a complete and detailed comparison of the libraries. Instead, its purpose is to highlight the fact that through comparing feature models, certain similarities and differences are easily picked up. This is particularly true if the feature models have been organized, at least approximately, around the same domain concepts. In other words, the notions of vertex, edge, graph representation and graph traversal should be common across the feature models at hand. Which is not to say those concepts will translate to the same features with the same feature values across the models. Quite on the contrary as the reader can notice: each feature model is indeed independent of the others while they share some conceptual framework.

In other words, independent feature models are useful but are not perfect for comparison since they don't necessarily have a unified conceptual viewpoint, e.g. for graph representations. Furthermore, we must humbly re-emphasize that our models are not complete by any means: the documentation of each library refers to a multitude of less important details that, for simplicity, we did not include in our work. But the incomplete independent models we have already are enough to make the point we want to make: a unified conceptual framework is necessary to have a more systematic approach to the comparison of libraries: each library should have its feature model re-expressed according to this unified conceptual framework. In other words, there is still too much apparently independent features in the non-integrated feature models: graph item types, graph representations, container types, etc. We need a deeper understanding of variability across the domain, we need an integrated feature model.... 


\section{The Integrated Model}

\subsection{About Integration}

From our experience, developing a feature model for each of these individual libraries is a necessary exercise if one is to understand variations between these libraries. But we have also learnt that such a task may be somewhat unproductive unless it ultimately yields an integrated conceptual framework for the domain at hand. In other words, obtaining a feature model for each library independently of other relevant libraries constitutes an intermediate task, not an end result: without some conceptual integration, it will be quite difficult to understand the similarities and differences between these libraries. We intend to emphasize this observation in this chapter by carrying out such integration and providing a much more detailed comparison of the libraries in light of this integration.

We have tried several approaches to come up with an integrated model. Here are some observations about the construction of an integrated model:

- At the very beginning, we tried to draw a domain model mainly out of text books, regardless individual libraries. The domain model we got is quite oversimplified.

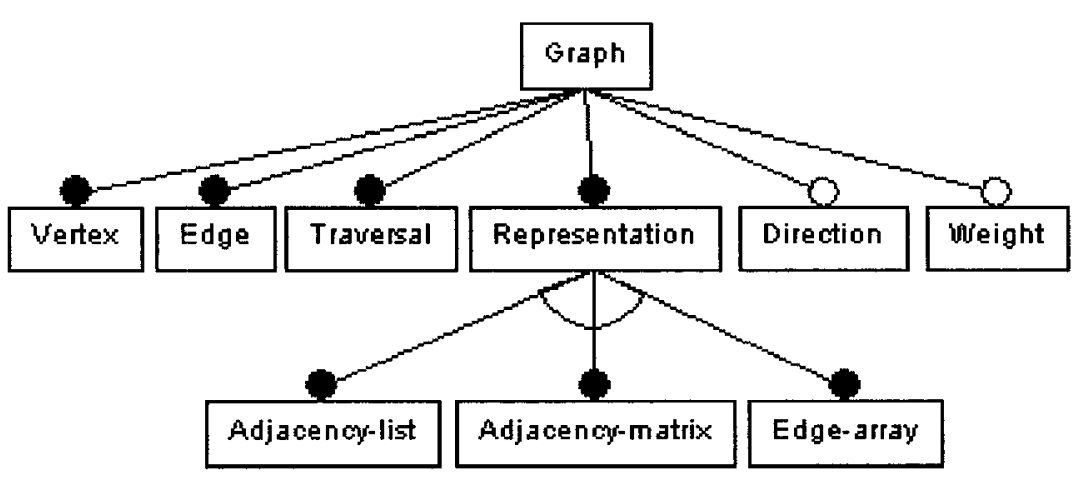

Figure 4-1 Feature diagram - Graph from textbooks 
Starting with this simple (if not simplistic) model, we tried to "fit" existing libraries in it. We found our simple model was poor and unstable. We had to change it every time we found a new idea from the individual library we are looking at. Here is an example of one such feature diagram that is quite incomplete.

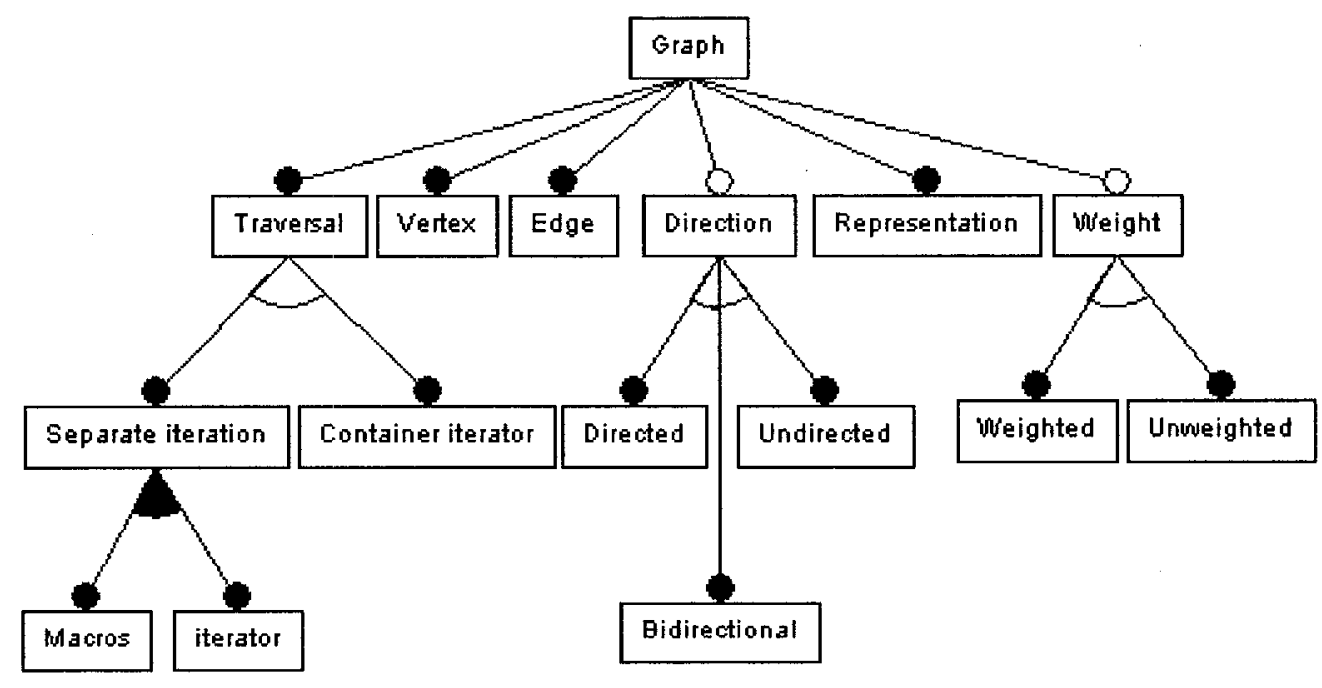

Figure 4-2 Feature diagram - Graph from textbooks (2)

In this feature diagram, we are trying to capture different kinds of traversal strategies provided by each library. We changed this feature every time we met a new implementation. Such a symptom clearly indicates a lack of conceptual analysis: one has to step back to understand variability.

- As shown in the previous chapter, we also tried to develop a feature model in vacuum for each existing library independently. In this case, comparison and integration can be arduous and must be followed by re-expressing each of these initial models using the integrated model. That is, each library must be shown to "fit" completely within the integrated conceptual framework. If the individual feature models are in fact developed independently of each other, then some will require a 
considerable amount of work to 'fit' in the integrated model.

- The approach we experimented last and strongly recommend is to first model the library that clearly offers (from the documentation) the most variability as a starting point and incrementally integrate the other libraries into this initial model. We found this approach more efficient than the one that develops feature models independently of each other and then tries (possibly painfully) to integrate them.

Following this last strategy, we start with BGL. Clearly, it has the most variation points including: selectable graph representation and selectable container type. And it abstracts out properties from graph and graph items. We quickly realize that this conceptualization can actually fit all other libraries. In other words, the most flexible library typically leads to conceptualizations that fill encapsulate several other libraries. But it must be emphasized that choosing the library that appears to be the most flexible one as a starting point for an integrated model does not guarantee that significant changes will not be necessary later.

The integration process is incremental and we suggest that it be not carried out per library but instead per feature. More precisely, we suggest that given the feature model for the most flexible library, integration be carried per feature of this model. For example, having obtained a feature model for BGL, we identified the following high-level features: vertex, edge, traversal, property and representation. These are the features under the root and define the broad categories for the conceptualization. What we are suggesting is that instead of choosing a next library to integrate into this initial feature model, it is preferable to choose a 
category (e.g., representation) and integrate all relevant libraries with respect to this category, and then and only then choose another category (e.g., traversal) and repeat the process. In other words, the very first feature model acts as a conceptual framework giving us the 'dimensions' on which to integrate across libraries.

Intuitively, the choice of the initial library, as well as the order in which the 'dimensions' of the integration will be considered do affect the amount of effort required for the integration, and the final result. But, at this point in time, we have no heuristics to offer to the reader to guide the selection of the initial library, nor the order of consideration of the dimensions.

Instead, we directly present our integrated model:

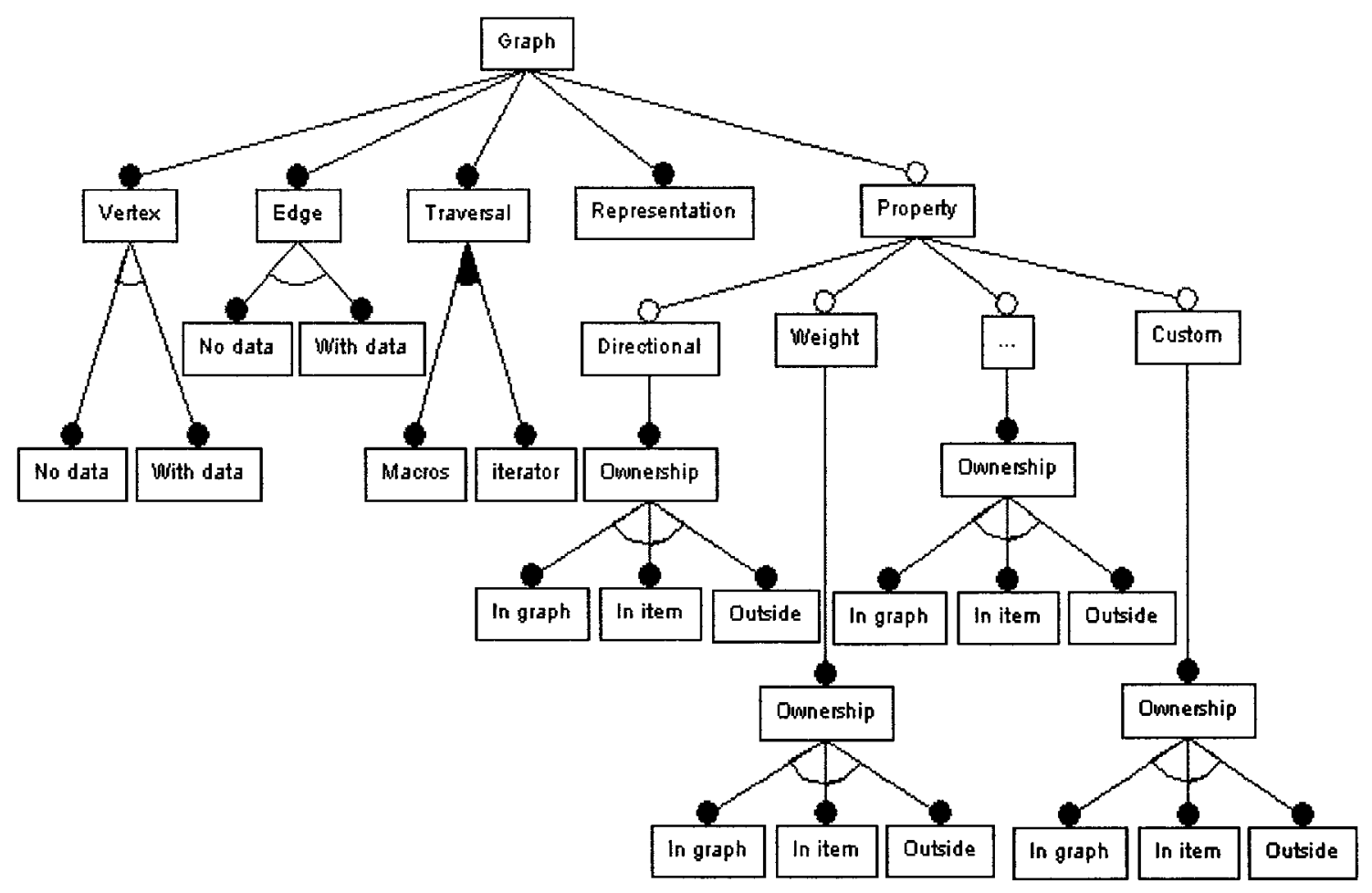

Figure 4-3.a Feature diagram - Graph 


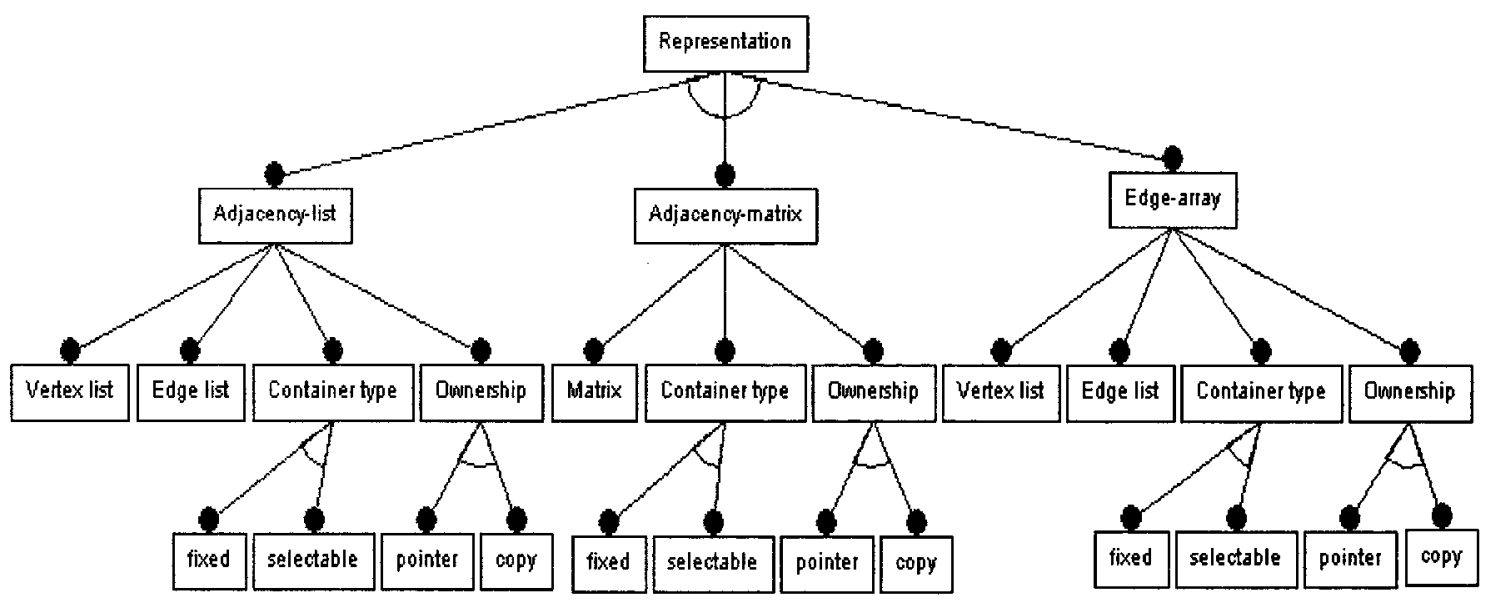

Figure 4-3.b Sub-feature - Graph Representation

Here is an explanation of the different features:

Feature Name: Vertex

A vertex (or node) is one of two basic notions of a graph. Some graph

libraries view a vertex as a container of information, whereas, for others,

it is merely a symbol-holder. In our model, we have abstracted these

details into two sub-features: withData and noData. Specific

implementations, such as the one of LEDA, would be captured as

sub-features of the withData feature. From this viewpoint, the integrated

model does act as a framework: further customization is possible by

decomposing the suggested leaf-nodes into more specific features.

Feature Name: Edge

The edge is the other fundamental notion in graphs. It may contain information, or not. Thus, we adopt the same decomposition strategy as for Vertex. In other words, Vertex and Edge share the similar sub-features. 
Feature Name: Representation

As previously mentioned, the adjacency list, adjacency matrix and the array of edges are generally used for the representation of a graph. A fourth sub-feature of Representation could have been used for custom implementations, but this seems to defeat the purpose of reusable components... Furthermore, we remark that each of the three typical implementation strategies is decomposable (as illustrated in Figure 4-3.b). What is interesting is that, in hindsight, all these implementations can be thought to share almost the same decomposition (different libraries making specific commitments in terms of feature values). We briefly elaborate on this decomposition of feature Representation across the implementation strategies: Every implementation strategy must somehow represent the vertices and the edges. We have found that this requires commitment to some type of Container (leading to the feature of the same name). Depending on the specific library, the user may or may not be given the ability to select this container type. Hence, the features fixed and selectable. A specific implementation must also decide if it stores its information using copies or using pointers. For this facet of the domain, we reuse the feature Ownership suggested by Czarnecki and Eisenecker $[11]^{12}$. Finally, each implementation defines an interface for accessing edges and vertices. Both Adjacency List and Edge Array offer access to a (possibly empty) set of vertices and a (possibly empty) set of edges.

\footnotetext{
12 We refer the reader to chapter 12 of that reference for an enlightening discussion of Lists, a form of sequence containers, but also for the motivation of the conceptual framework they offer and that we have tried to reuse as much as possible for graphs. The point here is that 'good' feature models can strongly guide the organization of other feature models!
} 
Conversely, an Adjacency Matrix offers access to a vertex matrix.

Feature Name: Traversal

Many algorithms rest on the ability to traverse a data structure. But traversal can be implemented in a multitude of different ways. Should each possibility be a sub-feature of this feature? We think not. In fact, traversal algorithms can be partitioned into two radically distinct kinds of solutions. LEDA provides a set of macros such as forall_edges(). All other libraries provide what are known as iterators [13]. Different kinds of iterators exist. For example, a graph class may supply its own traversal routines as part of its interface. These are called in-class iterators. Other libraries may reuse directly the iterator of their representation (e.g., use a List iterator, for example from STL, when their representation is an adjacency list). And some class may directly play or delegate to a helper class the role of an adapter, that is, a class that "translates" requests for traversal sent to the graph into their equivalent sequence of calls for the representation of this graph.

\section{Feature Name: Property}

The last feature of our top-level feature model for Graph is an optional one: the open set of properties relevant to graphs. In our opinion, it is this feature that illustrates the most the benefits of an integrated model. Let us briefly explain. Each library offers its conceptualization of a graph. In 
particular, each associates a set of properties to a graph. Such properties could be modeled as individual features at the top level. But this would make comparing the libraries more difficult than if we introduce the meta-concept of a property and "force" the domain engineer to "fit" properties found in individual libraries under this concept. In other words, introducing the feature Property has the effect of localizing the specificities of a graph representation under a common feature. A less desirable alternative is to compare libraries using feature models that can potentially conflate properties with other features. For example, Weight and Direction could be "buried" in features pertaining to Container type (e.g., UnweightedList, WeightedList, UnidirectionalList, BiDirectional-List, WeightedUnidirectionalList, etc.). Instead, Weight and Direction, which are highly reused notions across the libraries, are placed directly under Property. The sub-feature Custom of Property is used to localize in its sub-features those properties that are truly idiosyncratic to a particular library (i.e., non-reusable properties!). There is a difficulty, however, with feature Property: in libraries, properties are not systematically assigned (or "owned”) by the Graph. For example, in LEDA, both the Vertex and the Edge classes store properties. To reflect this, each property necessarily has an ownership sub-feature. We partition ownership of a property into three categories: properties owned by the graph, by graph items or by some other container outside graph and graph items. So, under feature InItem, we could have InVertex, and InEdge. Under feature Outside, we could have in InPropertyMap, and InTraversal, etc. This is still clumsy: what we 
really want is Ownership to be a parameterized feature (much like templates are parameterized classes in $\mathrm{C}++$ ). This idea is close in spirit to the work of Smaragdakis and Batory on mixin layers [12]: an important proposal for a systematic approach to the organization of features. This observation is important as it highlights the limitations of current feature model notations to deal with feature interaction, a key problem that we address elsewhere [42] (partly by reusing the key aspects of the notion of a mixin layer). That is, in our work, we are willfully restricting ourselves to the notation of [11]. Finally, more than any other feature, Property emphasizes the need for documenting carefully the feature combination rules (studies in details in [42]). For example, we could want to capture the rule that the property Direction must be owned by the class Graph, whereas a property Color (used for clustering algorithms) must be owned by the Vertex class. It is important to understand that the feature model notation does not necessarily capture all such rules. Indeed, separate rules are often necessary. The enumeration of such rules is not trivial: some rules hold across libraries, whereas others are specific to a library. In this dissertation, we have chosen to not use separate rules for feature combinations. More specifically, in order to facilitate the comparison of different libraries, we have tried to capture all such rules graphically, that is, explicitly in the individual feature diagram(s) we developed for each library from the integrated feature model presented here. 
Here is a table summarizing all features we use in the integrated model and how they map onto the different libraries we studied:

Table 4-1 Feature summary

\begin{tabular}{|c|c|c|c|c|}
\hline \multirow{2}{*}{ 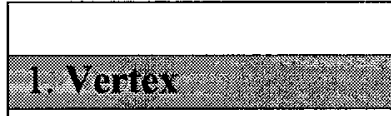 } & \multirow{2}{*}{ GTL-G } & \multirow[t]{2}{*}{ GTL-R } & \multirow{2}{*}{ BGL } & \multirow[t]{2}{*}{ LEDA } \\
\hline & & & & \\
\hline 1.1 No data & - & - & $\sqrt{ }$ & - \\
\hline 1.2 With data & $\sqrt{ }$ & $\sqrt{ }$ & - & $\sqrt{ }$ \\
\hline 2. Dage & (2) & (2) & 2 & (3) \\
\hline 2.1 No data & - & - & $\sqrt{ }$ & - \\
\hline 2.2 With data & $\sqrt{ }$ & $\sqrt{ }$ & - & $\sqrt{ }$ \\
\hline 3. Presentation & 8 & 8 & 58 & 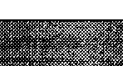 \\
\hline 3.1 Adjacency-list & $\sqrt{ }$ & $\sqrt{ }$ & $\sqrt{ }$ & $\sqrt{ }$ \\
\hline 3.1.1 Vertex list & $\sqrt{ }$ & $\sqrt{ }$ & $\sqrt{ }$ & $\sqrt{ }$ \\
\hline 3.1.2 Edge list & $\sqrt{ }$ & $\sqrt{ }$ & $\sqrt{ }$ & $\sqrt{ }$ \\
\hline 3.1.3 Container type & -3 & 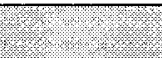 & 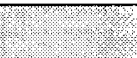 & 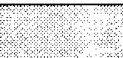 \\
\hline 3.1.3.1 Fixed & $\sqrt{ }$ & $\sqrt{ }$ & - & $\sqrt{ }$ \\
\hline 3.1.3.2 Selectable & - & - & $\sqrt{ }$ & - \\
\hline 3.1.4 Ownership & 3 & 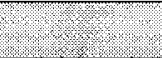 & 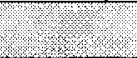 & $\bar{s}$ \\
\hline 3.1.4.1 Pointer & $\sqrt{ }$ & $\sqrt{ }$ & - & $\sqrt{ }$ \\
\hline 3.1.4.2 Copy & - & - & $\sqrt{ }$ & - \\
\hline 3.2 Adjacency-matrix & - & $\sqrt{ }$ & $\sqrt{ }$ & - \\
\hline 3.2.1 Matrix & & $\sqrt{ }$ & $\sqrt{ }$ & \\
\hline 3.2 .2 Container type & P: & s. & 1.: & 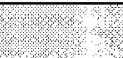 \\
\hline 3.2.2.1 Fixed & & $\sqrt{ }$ & $\sqrt{ }$ & \\
\hline 3.2.2.2 Selectable & & - & - & \\
\hline 3.2.3 Ownership & 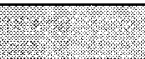 & 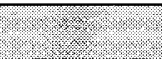 & 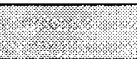 & (1) \\
\hline 3.2.3.1 Pointer & & $\sqrt{ }$ & - & \\
\hline 3.2.3.2 Copy & & - & $\sqrt{ }$ & \\
\hline 3.3 Edge-array & - & $\sqrt{ }$ & - & - \\
\hline 3.3.1 Vertex list & & $\sqrt{ }$ & & \\
\hline 3.3.2 Edge list & & $\sqrt{ }$ & & \\
\hline 3.3.3 Container type & & (3) & & be \\
\hline 3.3.3.1 Fixed & & $\sqrt{ }$ & & \\
\hline 3.3.3.2 Selectable & & - & & \\
\hline 3.3.4 Ownership & & . & & \\
\hline
\end{tabular}




\begin{tabular}{|c|c|c|c|c|}
\hline 3.3.4.1 Pointer & & $\sqrt{ }$ & & \\
\hline 3.3.4.2 Copy & & - & & \\
\hline \multicolumn{5}{|l|}{ 4. Traversal } \\
\hline 4.1 Macros & $\sqrt{ }$ & - & - & $\sqrt{ }$ \\
\hline 4.2 Iterator & $\sqrt{ }$ & $\sqrt{ }$ & $\sqrt{ }$ & $\sqrt{ }$ \\
\hline \multicolumn{5}{|l|}{ S. Properity } \\
\hline \multicolumn{5}{|l|}{ 5.1 Ownership } \\
\hline 5.1.1 In graph & $\sqrt{ }$ & $\sqrt{ }$ & $\sqrt{ }$ & $\overline{\sqrt{ }}$ \\
\hline 5.1.2 In item & $\sqrt{ }$ & $\sqrt{ }$ & - & $\sqrt{ }$ \\
\hline 5.1.3 Outside & $\sqrt{ }$ & & $\sqrt{ }$ & \\
\hline 5.2 Direction & $\sqrt{ }$ & $\sqrt{ }$ & $\sqrt{ }$ & $\sqrt{ }$ \\
\hline 5.3 Weight & $\overline{\sqrt{ }}$ & $\sqrt{ }$ & $\overline{\sqrt{ }}$ & $\sqrt{ }$ \\
\hline 5.4 Custom & - & $\sqrt{ }$ & $\sqrt{ }$ & $\sqrt{ }$ \\
\hline
\end{tabular}

Now let us see how each library can "fit" in the integrated model (with changes in bold)

\subsection{GTL-G revisited}

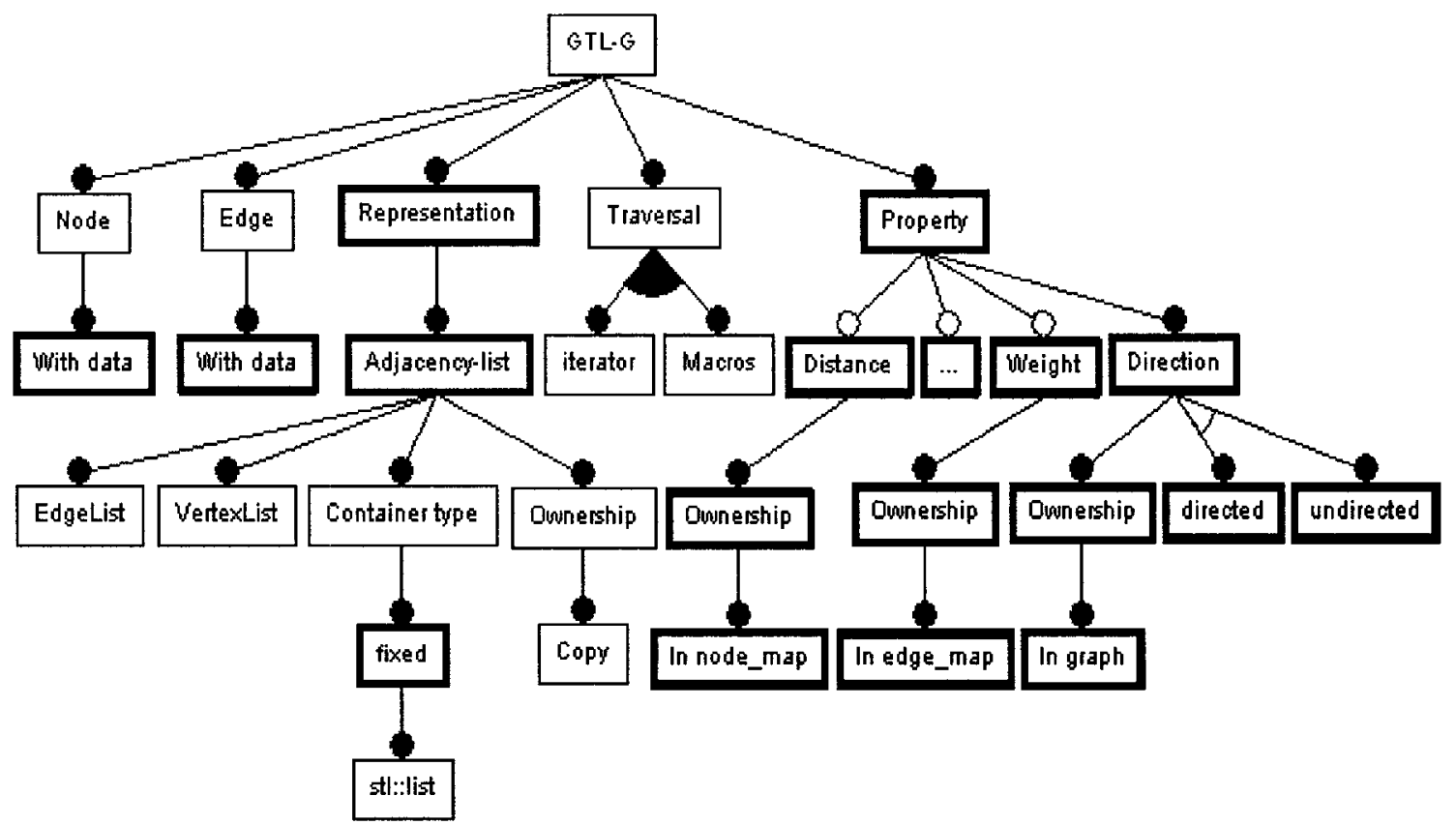

Figure 4-4 Feature diagram - GTL-G after integration 
In our opinion, the integrated model can accommodate GTL-G very well. For example, no feature needs to be dropped. And, in fact, we believe that some design decisions of GTL-G are made more explicit. For example, the fact that both node and edge have some information associated with them. Also, clearly GTL-G has implemented only the adjacency_list as graph representation and uses a fixed container type. And, the use of feature Property greatly helps understanding a key facet of this library, namely that maps are used to hold properties. We believe such a detail being understood upfront will greatly reduce the time required to understand the code.

\subsection{GTL-R revisited}

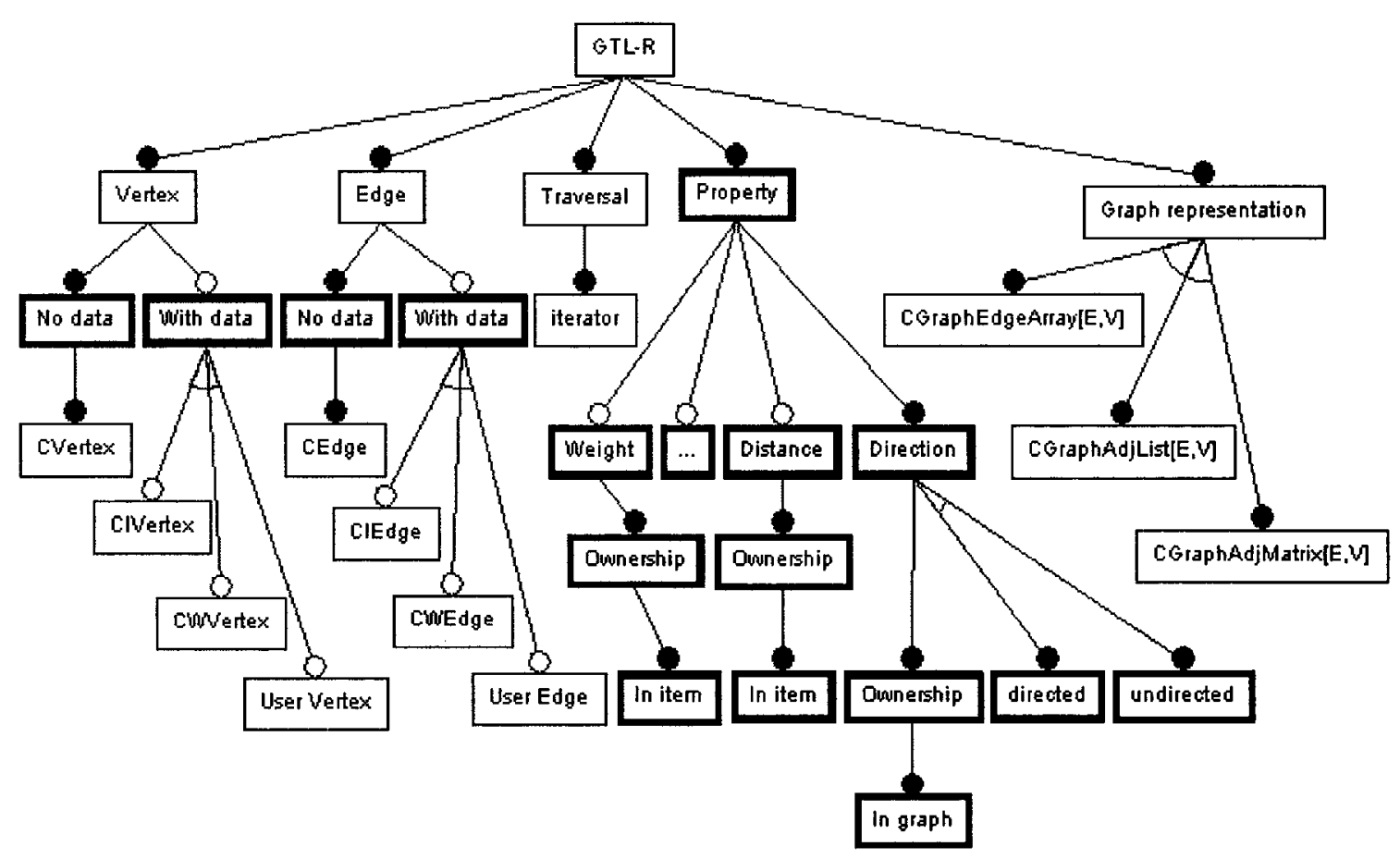

Figure 4-5.a Feature diagram - GTL-R after integration 


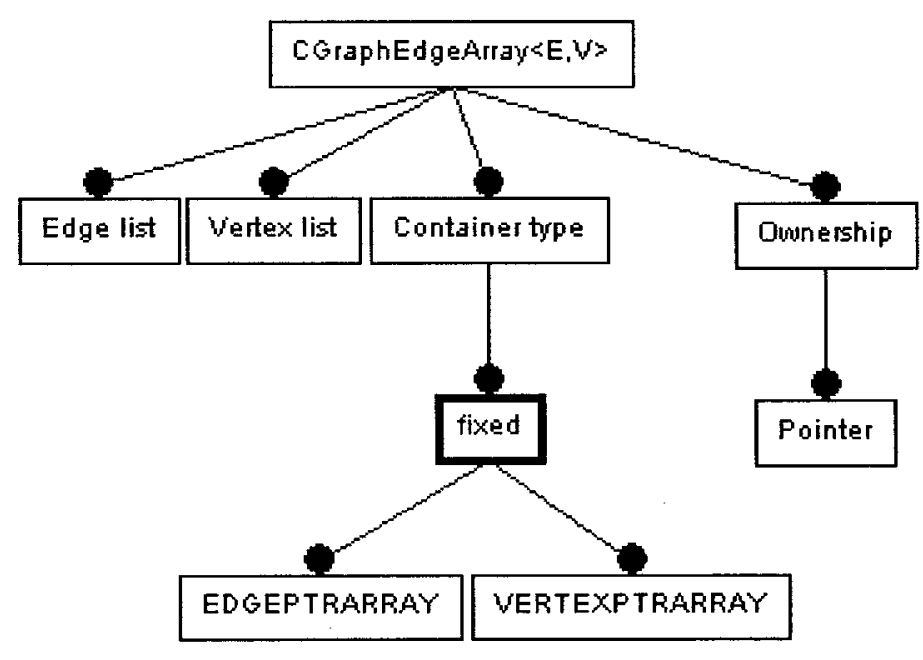

Figure 4-5.b Sub-feature - edge array under representation in GTL-R

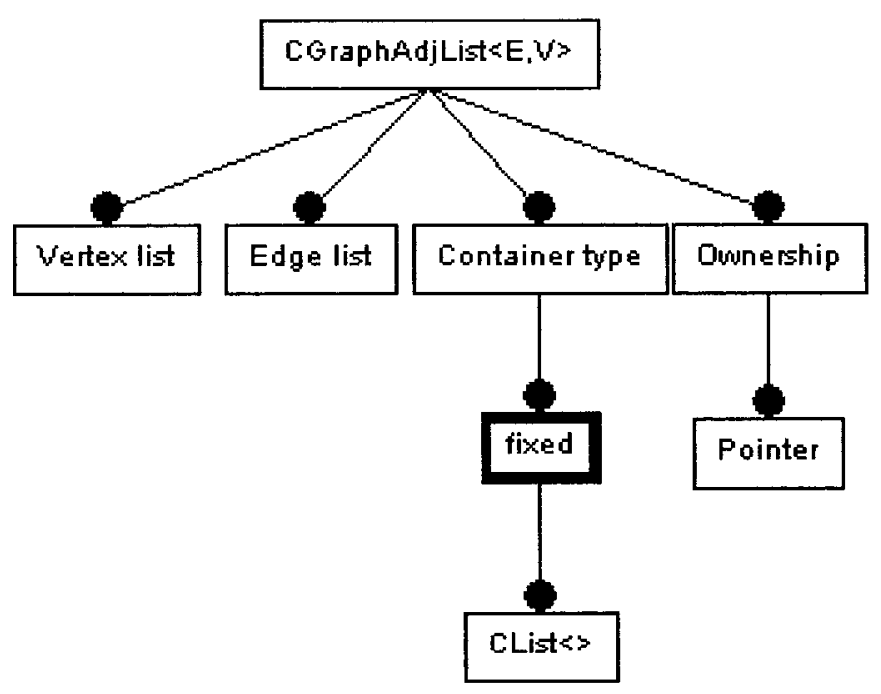

Figure 4-5.c Sub-feature - adjacency list under representation in GTL-R 


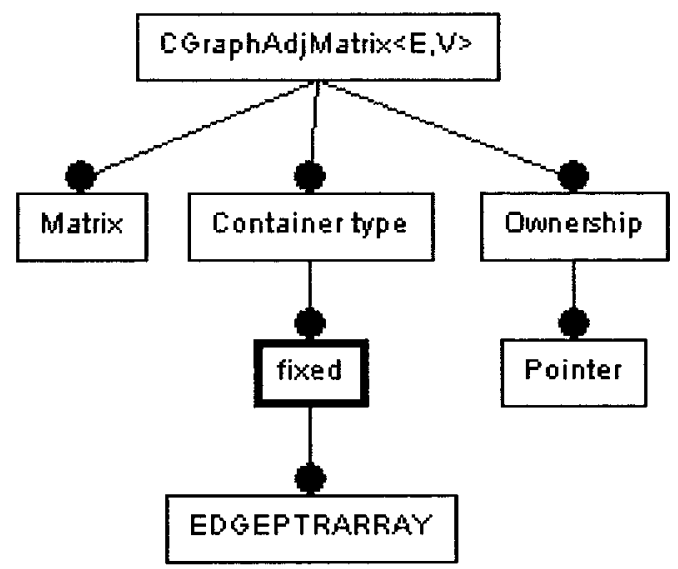

Figure 4-5.d Sub-feature -

adjacency matrix under representation in GTL-R

GTL-R also fits quite easily the proposed integrated feature diagram. The diagrams show that vertex and edge in GTL-R can have data or not. No data is mandatory feature while With data is an optional one.

Feature property again is essential in structuring the understanding of this library. In particular, we can see that properties of graph items are stored in these items themselves. 


\subsection{BGL revisited}

Please recall that this is the library used to build the initial version of our integrated model.

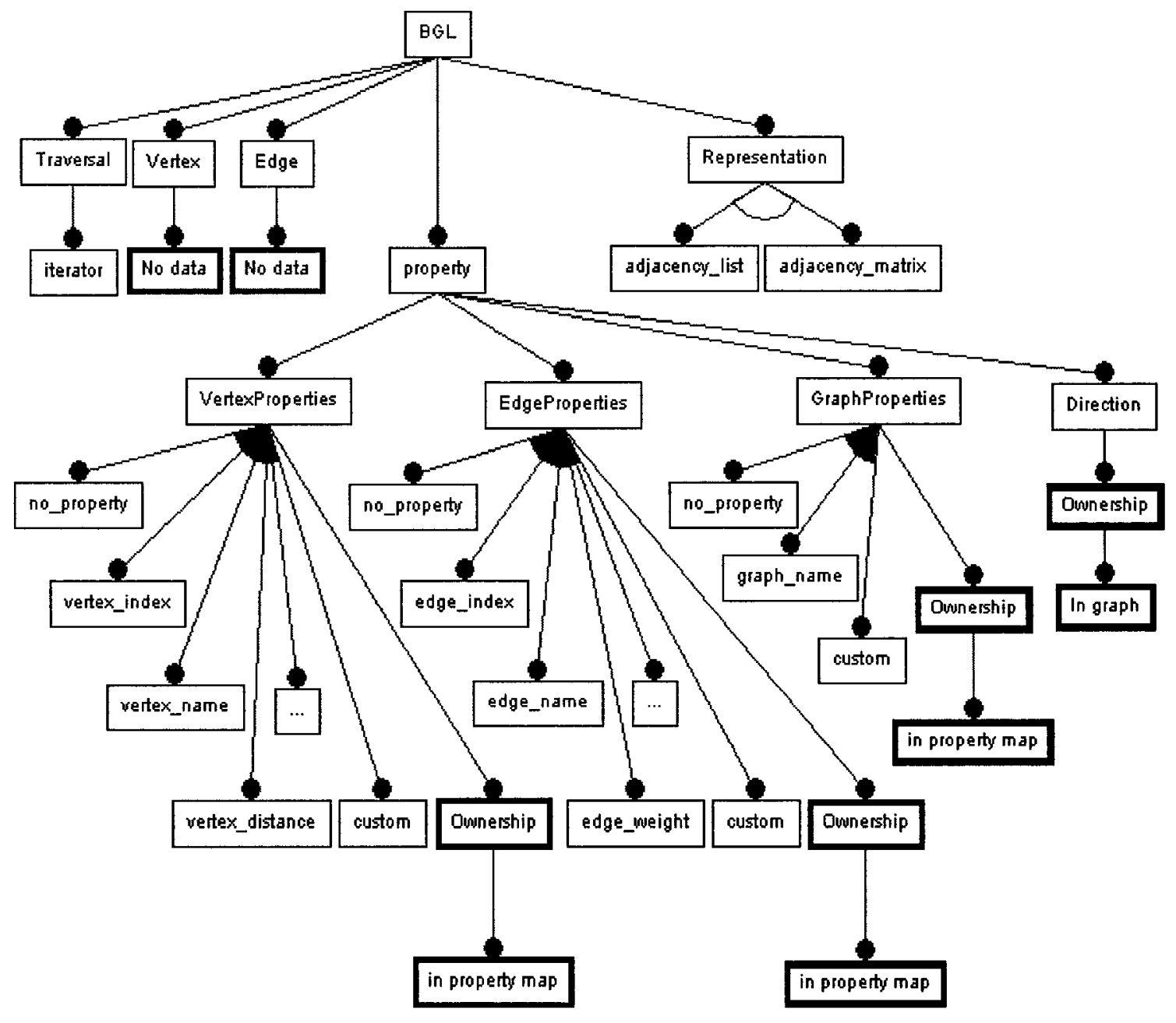

Figure 4-6.a Feature diagram - BGL after integration 


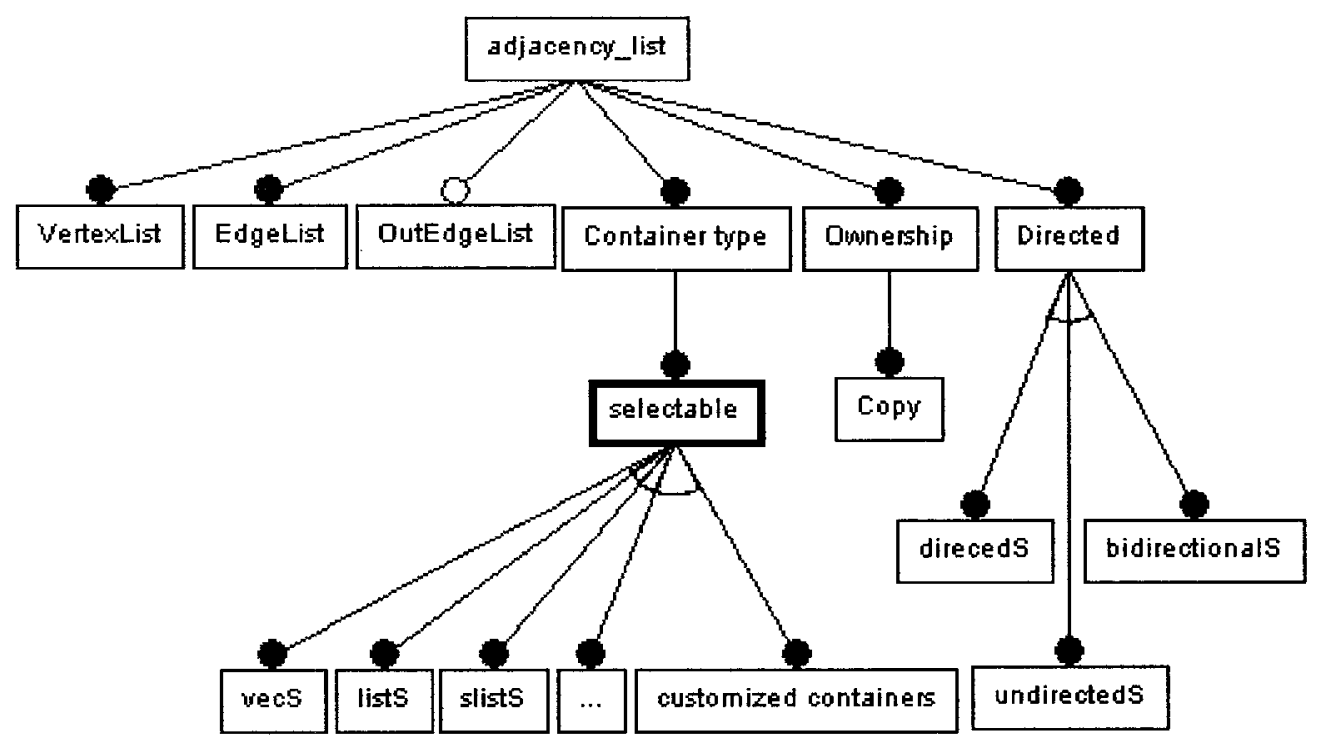

Figure 4-6.b Sub-feature - adjacency list under representation in BGL

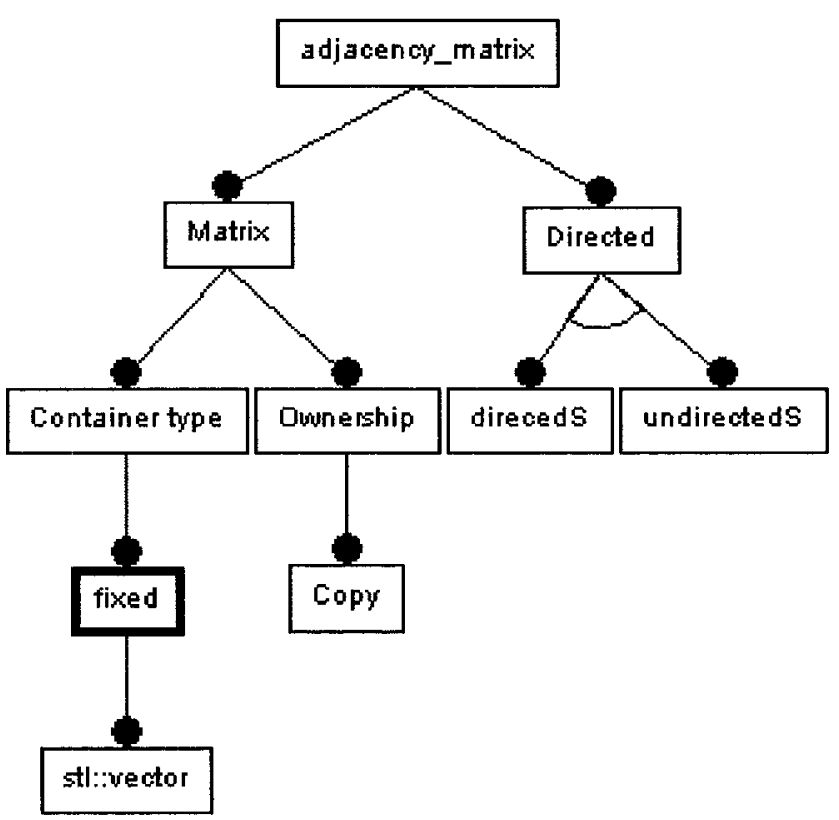

Figure 4-6.c Sub-feature - adjacency matrix under representation in BGL

It is important to notice that it is not because a library acts as the starting point for integration that it cannot have some of its conceptualizations abandoned. 
For example, we dropped feature Allocator from BGL-specific model because it is extremely language specific and truly an idiosyncrasy of BGL.

In our opinion, the new model for BGL is easier to understand than the one presented in the previous chapter (and this comment applies in fact to all libraries). We can see from it, that features vertex and edge have No data as a sub-feature. Also, clearly, BGL implements two graph representations (adjacency list and adjacency matrix). And it separates properties into three groups: vertex properties, edge properties and graph properties, and stores them in property maps. Also, the container type is selectable in adjacency list, whereas it is fixed in adjacency matrix. Finally, there is an extra container to store out-edges for an adjacency list if the graph is bidirectional. 


\subsection{LEDA revisited}

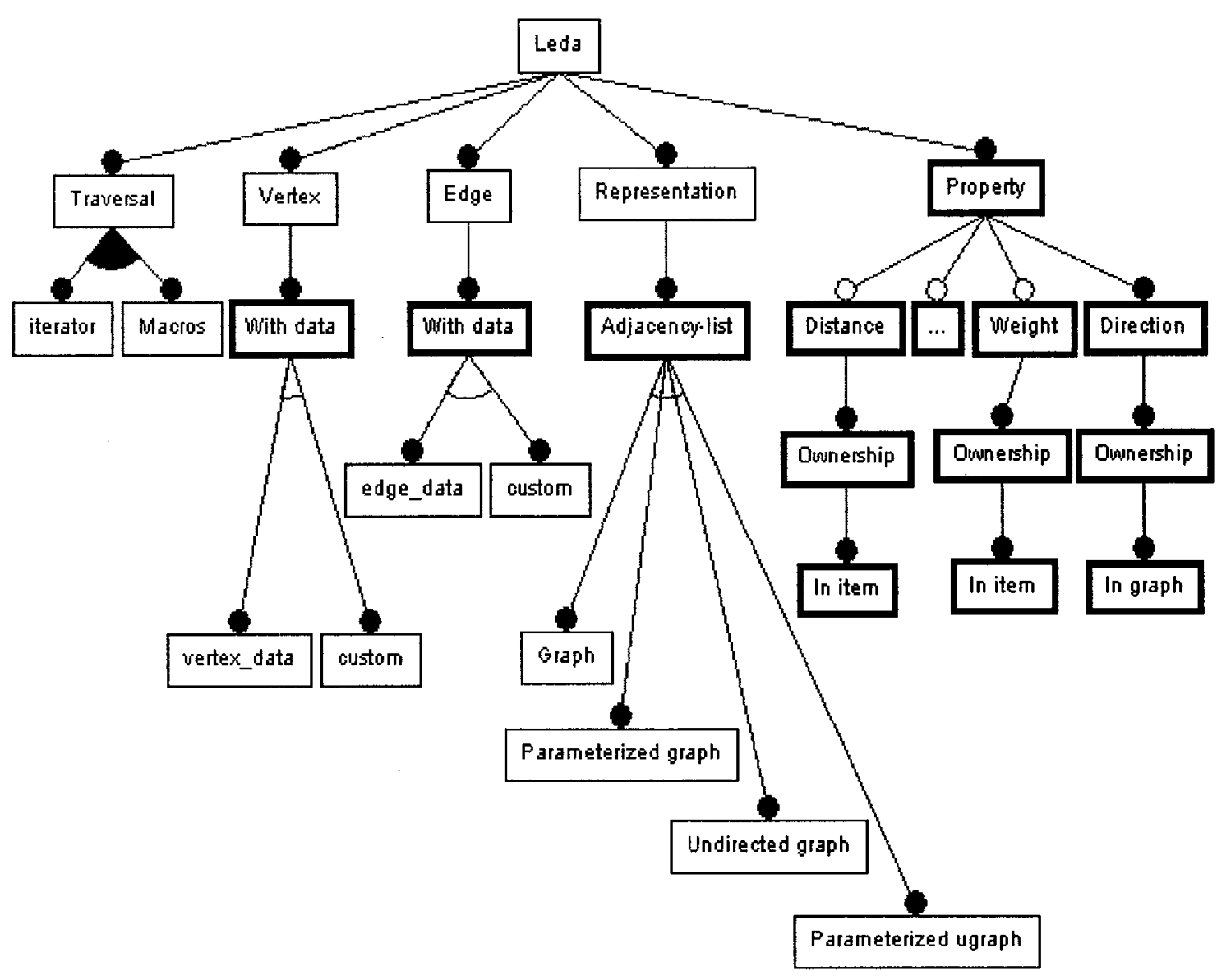

Figure 4-7.a Feature diagram - LEDA after integration

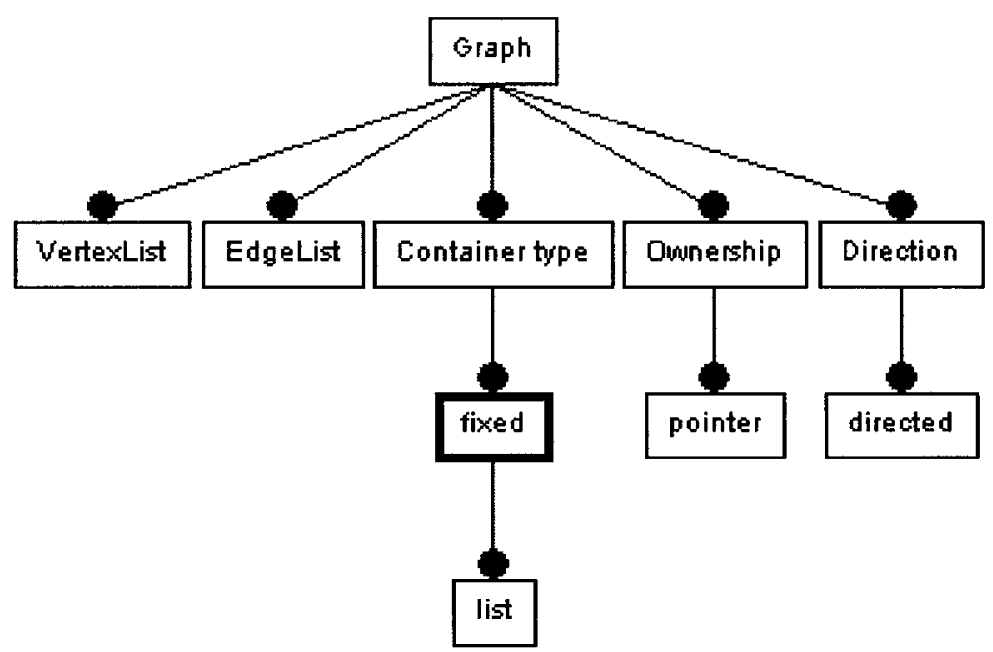

Figure 4-7.b Sub-feature - graph under representation in LEDA 


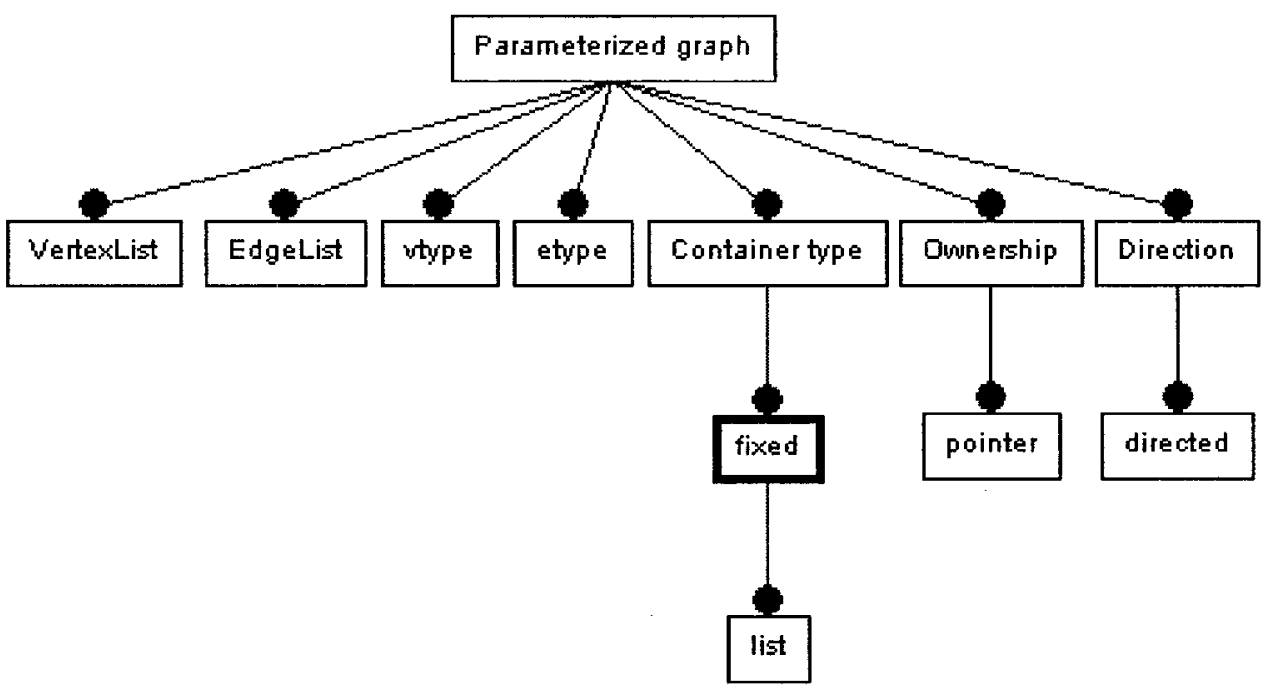

Figure 4-7.c Sub-feature - parameterized graph under representation in LEDA

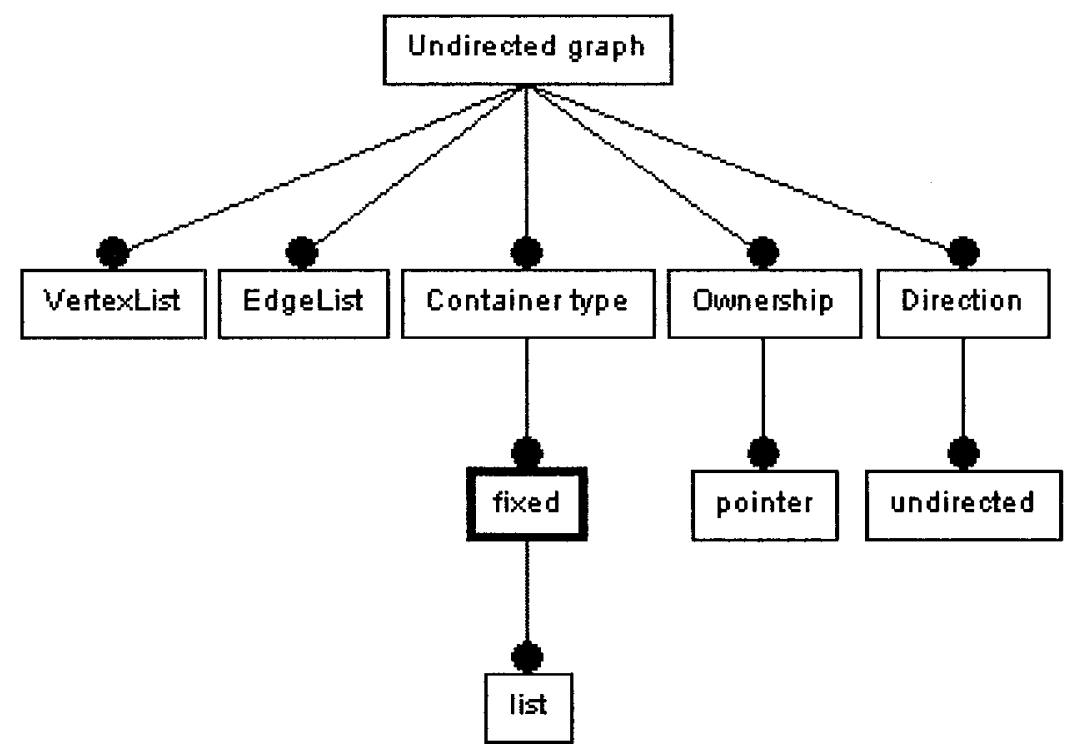

Figure 4-7.d Sub-feature - undirected graph under representation in LEDA 


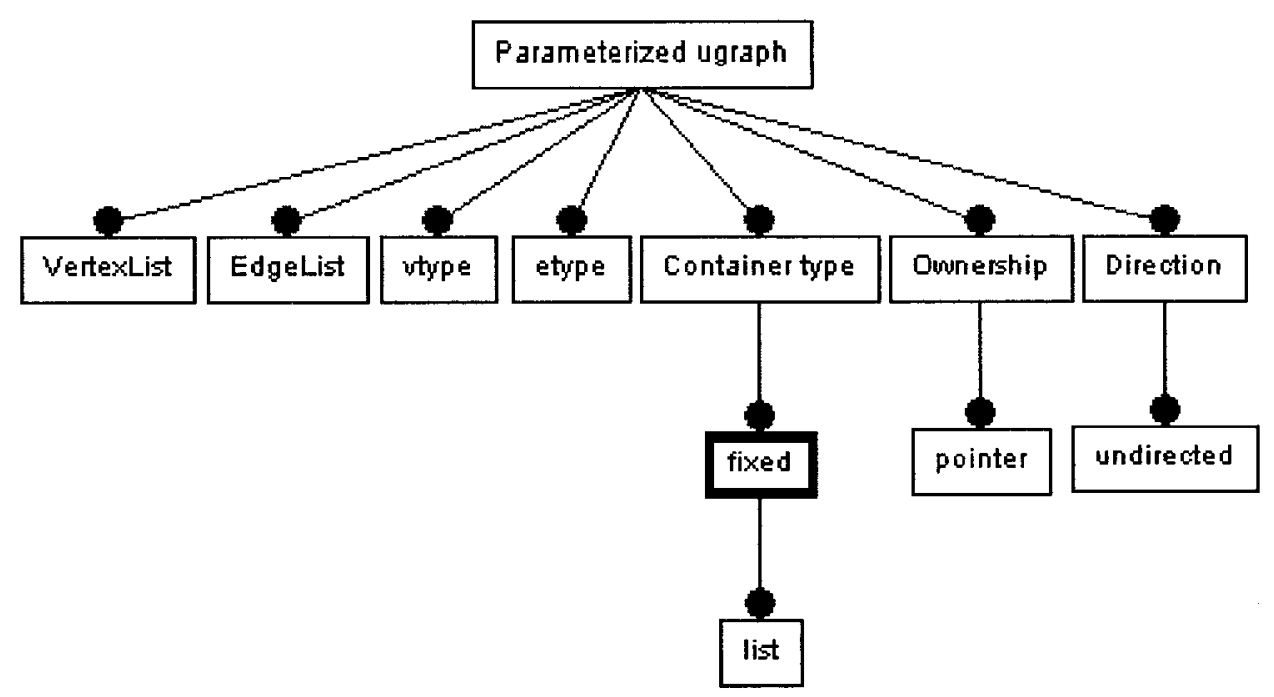

Figure 4-7.e Sub-feature - parameterized ugraph under representation in LEDA

We observe that again, the library at hand, in this case LEDA, "fits" easily into the proposed integrated model. We can see that Edge and Vertex are captured using with data. The data can be built-in or customized. Furthermore, though only one graph representation is supported (namely, adjacency list), four types of graph are available. All four graph types use fixed containers to store graph items. And, properties of graph items are stored in items.

\subsection{Library selection}

In the graph domain implicitly defined by the graph libraries we have studied, variability originates mainly from five aspects:

- Whether or not information is associated with edges, and with nodes,

- Where to store properties,

- Which graph representation to use,

- Whether the container type is fixed or selectable,

- What is the ownership of the container. 
Here is a summary for those variation points for individual libraries:

Table 4-2 Main Variation points

\begin{tabular}{|l|l|l|l|l|l|l|}
\hline & Vertex & Edge & $\begin{array}{l}\text { Property } \\
\text { Owner-ship }\end{array}$ & Representation & Container & $\begin{array}{l}\text { Container } \\
\text { Ownership }\end{array}$ \\
\hline GTL-G & With data & With data & Outside & Adjacency-list & Fixed & Copy \\
\hline GTL-R & $\begin{array}{l}\text { With data } \\
\text { No data }\end{array}$ & $\begin{array}{l}\text { With data } \\
\text { No data }\end{array}$ & In item & $\begin{array}{l}\text { Adjacency-list } \\
\text { Adjacency-matrix } \\
\text { Edge-array }\end{array}$ & $\begin{array}{l}\text { Fixed } \\
\text { Fixed } \\
\text { Fixed }\end{array}$ & Pointer \\
\hline BGL & No data & No data & Outside & $\begin{array}{l}\text { Adjacency-list } \\
\text { Adjacency-matrix }\end{array}$ & $\begin{array}{l}\text { Selectable } \\
\text { Fixed }\end{array}$ & Copy \\
\hline LEDA & With data & With data & In item & Adjacency-list & Fixed & Pointer \\
\hline
\end{tabular}

This table is important and proceeds directly from the integrated feature model and its specialization for each library. In choosing a library, we believe user requirements must be analyzed and mapped onto this table. For example, should we need to support adjacency matrices, we only have two choices. Storing pointers or copies is another crucial decision that can be taken early.

Ultimately, this table in combination with the integrated feature model for each library can be used to derive a set of guidelines for choosing a library over another in light of a set of specific requirements. For example, LEDA uses a list to store items. If we need to add and remove graph items quickly, LEDA is a good choice. But, space requirements are larger for LEDA than for GTL-R, since GTL-R uses arrays to store items. Such analysis stems directly from careful study of the integrated feature models. Here is another more direct example. If we need to change graph representations at run time, then the table above clearly suggests that BGL and GTL-R are good choices. And if we need to change container types, BGL is the only choice. 
Given the integrated feature models we have developed to 'fit' each library we came up with the following high-level comments for each library. Such comments are meant to act as an "executive summary" to help in the choice of libraries.

\section{GTL-G:}

GTL-G is simple to use. It has "full size" graph items: much information is associated with the latter. This makes GTL-G has low efficiency. It has only implemented the adjacency list as graph representation. It uses lists from STL to store graph items. Everything is fixed in GTL-G, which makes it is simple to use. Flexibility is very limited. For those who don't care much about efficiency and flexibility, GTL-G is a good choice.

GTL-R:

GTL-R has both vertex type and edge type selectable on demand. This makes GTL-R efficient since it keeps unnecessary information outside applications. It implements three graph representations. This gives GTL-R excellent flexibility. Also, it uses a fixed container type (array or list) from MFC. For those who don't want to use containers from STL, GTL-R is the only choice in the domain we are considering.

GTL-R is also the only one that doesn't use outside storage such as maps to keep properties. Properties pertaining to graph items are stored inside these items on demand. This design decision makes the processing of such properties both simple and efficient. It provides interfaces (flavour classes) to access these properties. There are merely get and set methods inside flavour classes. 
BGL:

Flexibility is BGL's essential characteristic. It has simple graph items, but can capture their variations through properties. It puts all information about graph items into maps. This leads to some inefficiency with respect to performance. Also, it offers two graph representations. But the most important characteristic is that it has selectable container type, which makes it stand out from other libraries. For those who want to use different container types, BGL is the only choice.

\section{LEDA:}

LEDA is simple to use, and, as previously mentioned, close to textbook style. But it has poor efficiency because of its "full size" vertex class and edge class: all information about items is kept, whether needed or not. This information can not be accessed directly. Maps are used as interfaces to access this information. LEDA has limited flexibility. It uses solely the adjacency list to represent a graph. Also, it has a fixed container type: the list container from LEDA. So, in summary, it's only variation point is its user-defined vertex data and edge data.

Beyond such executive summaries, it is useful to carry out a qualitative analysis of each library with respect to the most frequent non-functional (often called "quality of service") requirements relevant to users of such libraries.

We carried out such an analysis and summarize our findings in the next table. 
It is important to add, however, that this analysis is somewhat subjective and that it would be preferable to carry out a quantitative analysis. The latter lies beyond the scope of this dissertation and is briefly discussed in the last chapter.

Table 4-3 NFR comparison

\begin{tabular}{|c|c|c|c|}
\hline & Flexibility & Simplicity & Efficiency \\
\hline GTL-G & 4 & 1 & 3 \\
\hline GTL-R & 2 & 3 & 2 \\
\hline BGL & 1 & 4 & 1 \\
\hline LEDA & 3 & 2 & 4 \\
\hline
\end{tabular}

\subsection{Prototyping a Generator}

We have prototyped a graph generator for our graph domain. We will briefly overview it here.

First we define the feature vocabulary for specifying graphs.

Some features defined in Figure 4-3 are represented by using enumeration types:

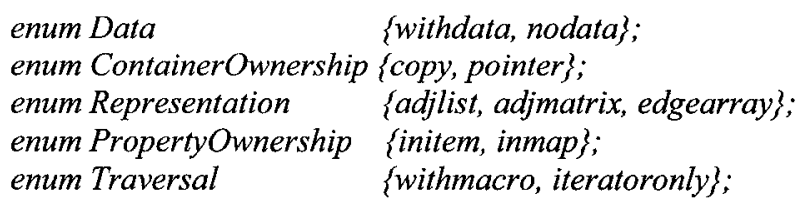

The feature Container type is captured as selectable in the generator. That is, we use a selector (this idea comes from BGL) to decide the container being used.

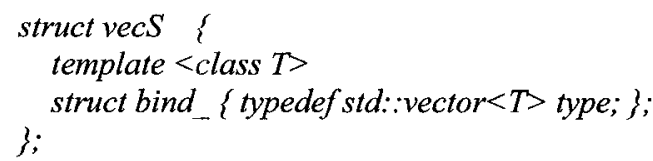

Selectors are also used to specify properties: 
Here is the top-level organization of the generator:

// template parameters include all features being used, with default values template $<$

//VertexList and Edgelist have default value list
class VertexList
$=$ lists;

class EdgeList

$=$ lists;

//Data is used to represent the implementation of the vertex and the edge. //They are implemented with withdata as default.

Data data $=$ withdata;

//The feature Container ownership. Copy is the default.

ContainerOwnership containerownership = copy;

//The feature Representation. It has three values to choice. Adjlist is the //default

Representation $\quad$ representation adjlist;

//The property related to vertices, not necessarily stored in Vertex

class VertexProperty_ = nopropertyS

//The property related to edges, not necessary stored in Edge

class EdgeProperty_ $\quad$ = nopropertyS;

//The feature Property ownership, properties are stored in items by defaullt

PropertyOwnership $\quad$ propertyownership = initem;

//The feature Traversal. It's default value is iteratoronly

Traversal traversal = iteratoronly;

//The direction is also decided by selectors

class Direction

$=$ undirectedS

$>$

class GRAPH_GENERATOR \{

public:

typedef GRAPH_GENERATOR<

VertexList

EdgeList,

data,

containerownership,

representation

VertexProperty,

EdgeProperty,

propertyownership,

traversal,

Direction_> Generator; //call it just a Generator

private: // capture at compile time the nature of the current generator

enum \{

is WithData $=$ data $==$ withdata,

isPointer = containerownership $==$ pointer,

isMap = propertyownership==inmap

isIterator $=$ traversal $==$ iteratoronly $\}$; 
We use meta-control structures [11, chapter 10], to select which components to reuse. For example, if the Vertex is required to store some information and the property is stored in the map, the Vertex will be implemented like the Vertex in GTL-G. If the Vertex is required to store some information and its properties are also stored in it, them we use a Vertex class similar with that in Leda.

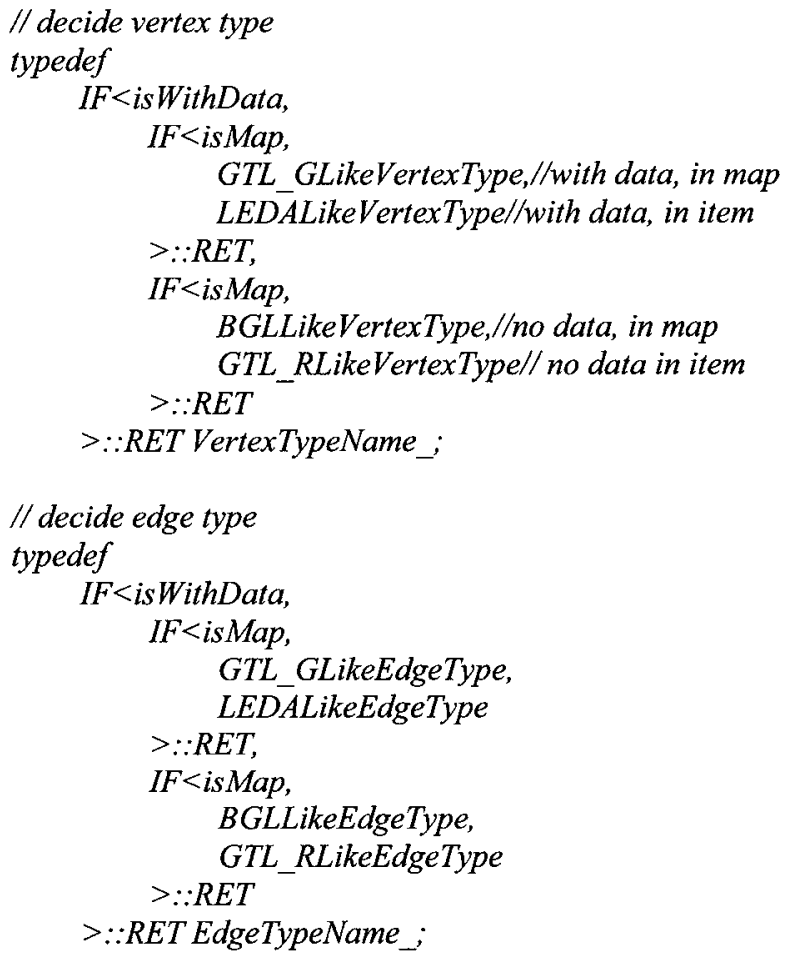

If container ownership is captured as Pointer, the item type will be a pointer to an item object. If instead captured as Copy, the item type will be an object of item classes.

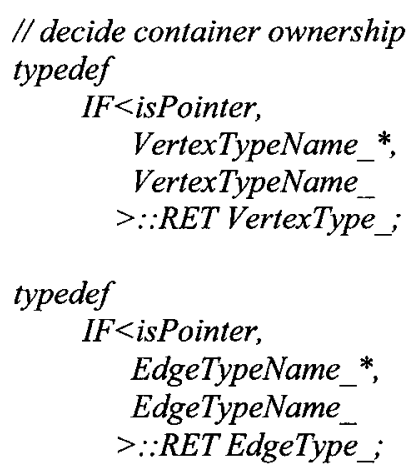

Here is the rest of such rules:

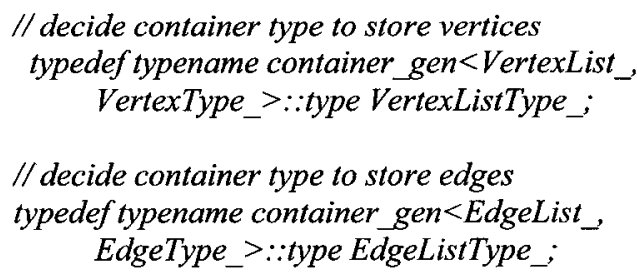


// decide property ownership

typedef

IF $<$ is Map,

// using map to store properties

PropertyMap <VertexTypeName, VertexProperty_>,

// no map used

IF $<$ isWithData,

// no map, with data --> like LEDA but without use of map

PropertyInItemData $<$ VertexTypeName_, VertexProperty_>,

// no map, no data in items --> like GTL-R

PropertyInFlavor<VertexTypeName, VertexProperty_>

$>\because R E T$

$>: \because R E T$ VertexPropertyType ; // vertex property

typedef

IF $<$ isMap,

PropertyMap $<$ EdgeTypeName, EdgeProperty_>,

IF $<$ isWithData,

PropertyInItemData $<$ EdgeTypeName, EdgeProperty_>, $>\because R E T$

PropertyInFlavor $<$ EdgeTypeName, EdgeProperty_>

$>:: R E T$ EdgePropertyType_// edge property

// decide iterator types

typedef

IF $<$ islterator,

IteratorOnly $<$ VertexTypeName_>,

MacroAndIter<VertexTypeName >

$>: \because R E T$ VertexIteratorType_;

typedef

$$
\begin{aligned}
& \text { IF }<\text { isIterator, } \\
& \text { IteratorOnly }<\text { EdgeTypeName_>, } \\
& \text { MacroAndIter }<\text { EdgeTypeName_> } \\
& >:: \text { RET EdgeIteratorType; }
\end{aligned}
$$

// decide direction

typedef typename Direction_: $:$ is_bidir_t $t$ is_bidir_; // is bidirectional or not typedef typename Direction_: $: \overline{i s}$ directed $t$ is_directed ; // is directed or not

// decide graph representation typedef

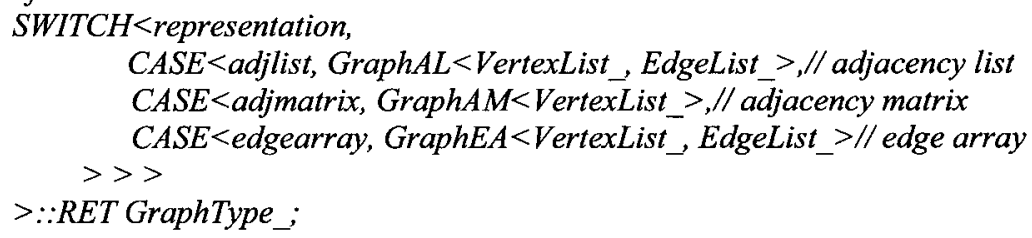

public:

typedef Graph $<$ Generator $>$ RET; 
Finally, this is the configuration structure that holds all the information about the graph classes being generated.

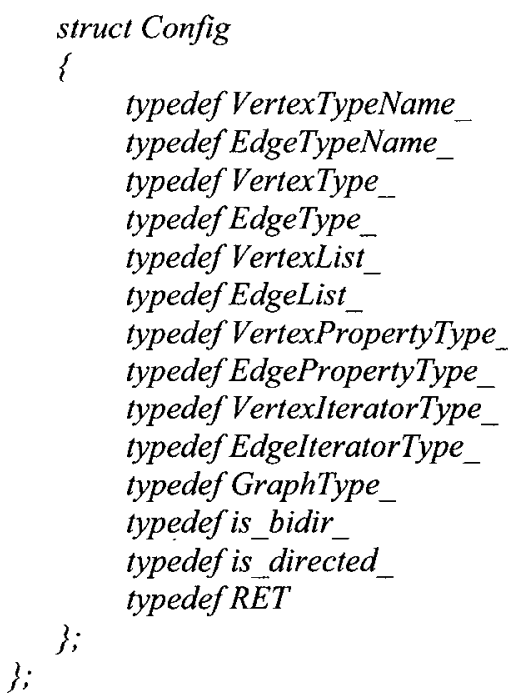

VertexTypeName; EdgeTypeName; VertexType; EdgeType; VertexList; EdgeList; VertexPropertyType; EdgePropertyType; VertexIteratorType; EdgeIteratorType; GraphType; is_bidir; is directed; ReturnType;

Writing a generator is quite straightforward if we have an integrated feature model for the domain since the generator proceeds directly from the feature model. What we need to do is to decide on the template list for the graph following our integrated feature model, and then decide corresponding components according to the relevant parameter values. 


\section{Looking for Tests}

\subsection{State-based Testing?}

Binder discusses at length the testing of generic containers in [17]. In essence, his strategy reduces to the design of an extremely general (somewhat simplistic) finite state machine and to the tests that result from it (through the application of some standard method to generate tests out of a state machine). His motivation can be summarized in the following quote [Ibid.]:

A state machine is a system whose output is determined by both current and past input. The effect of previous inputs is represented by a state[...] Object-oriented software is well suited to state-based testing [...] Although the number of message sequences and instance variable value combinations is essentially intractable for practical purposes, a state machine can nevertheless provide a compact and predictable model of behaviour[...] State machines are an effective, fundamental technique for design and test of complex behaviour.

Binder recommends two patterns to test a generic container class:

Category-Partition and Nonmodal Class Test. In Category-Partition, the C++ member function List::getNextElement( $)$ is used to illustrate development of a test suite. The test suite is produced in seven steps [Ibid]:

1. Identify the functions of the MUT (method under test).

2. Identify the input and output parameters of each function.

3. Identify categories for each input parameter.

4. Partition each category into choices.

5. Identify constraints on choices.

6. Generate test cases by enumerating all choice combinations

7. Develop expected results for each test case using an appropriate oracle. 
Table 5-1 shows the categories for the getNextElement( ) parameters.

Table 5-1 Categories for getNextElement( )

\begin{tabular}{|l|l|}
\hline Parameter & Category \\
\hline $\begin{array}{l}\text { Position of the last reference } \\
\text { element }\end{array}$ & $\begin{array}{l}n \text {th element } \\
\text { special cases }\end{array}$ \\
\hline State of the list & $\begin{array}{l}\text { m-elements } \\
\text { Special cases }\end{array}$ \\
\hline
\end{tabular}

Table 5-2 shows choices for the getNextElement() parameters.

Table 5-2 Categories and Choices for getNextElement( )

\begin{tabular}{|l|l|l|}
\hline Parameter & Category & Choices \\
\hline $\begin{array}{l}\text { Position of the last referenced } \\
\text { element }\end{array}$ & $n$th element & $\begin{array}{l}n=2 \\
n=\text { some } \mathrm{x}>2, \mathrm{x}<\operatorname{Max} \\
n=\text { Max } \\
\text { undefined } \\
\text { First } \\
\text { Last, } n<\text { Max }\end{array}$ \\
\hline State of the list & $\begin{array}{l}m=\text { some } \mathrm{x}>2, \mathrm{x}<\operatorname{Max} \\
\text { Empty } \\
\text { Singleton } \\
\text { Full }(m=\operatorname{Max})\end{array}$ \\
\hline
\end{tabular}

The test cases are identified by generating the cross-product of all the choices.

With categories of 6 and 4 choices, there are $24=6 \times 4$ test cases. [17]

In a sister dissertation [31], Juemin Yu provides an integrated feature diagram for the domain of sequential containers. She then extends the work of Binder to that domain, and develops a state machine that 'fits' the different containers she supports. From this state machine, she finally extracts tests for her containers.

What is interesting in Binder's and Yu's work with respect to our own is that graphs could be thought of as containers: graphs store items and provide methods to search for them. But, trying to develop of generic state machine for the different 
graphs supported by our libraries turns out to be a dead end: graphs are not containers, at best they use containers as their underlying representations. We are interested in how to develop tests for graphs per se and thus abandoned the strategy of trying to view graphs as containers.

In another dissertation of our research group [39], Wei Shi provides an integrated feature model for a family of algorithms dealing with leader election in a ring. Interestingly enough, she also developed an integrated state machine for the nodes of the ring. While this is useful for generating tests for a node (using Binder's strategies for test case generation out of state machines [17, chapter 7]), it does not help with the testing of her specific algorithm across the ring. In other words, it appears that testing the behavior of a node of the ring does not suffice to test the leader election algorithm per se (as the topology itself has to be tested). Similarly, developing a state machine for a node of a graph:

1) makes very little sense outside of the context of a specific algorithm. But we have purposely chosen to model the domain of graphs independently of the algorithms that can be performed over graphs.

2) is not sufficient to test the algorithm per se.

The point to be grasped is that our investigation suggests that state machines are not a promising starting point for the derivation of tests for the domain of graphs. Graphs should not be viewed as data structures per se but as "customers" of such data structures (handled under feature representation and testable by themselves, that is, independently of their use in graphs or other applications. 


\subsection{Decision Trees}

Binder also discusses at length the use of decision trees to generate test cases using combinational models [17]. He adds that a decision tree is indicated as a test model when the implementation under test has the following characteristics [Ibid]:

- One of several distinct responses is to be selected according to distinct cases of input variables.

- These cases can be modeled by mutually exclusive Boolean expressions on the input variables.

- The response to be produced does not depend on the order in which input variables are set or evaluated.

- The response to be produced does not depend on prior input or output.

Following this idea, we reinterpret a part of our integrated feature diagram as a decision tree (illustrated in figure 5-1), much like [11] does for a simple list.

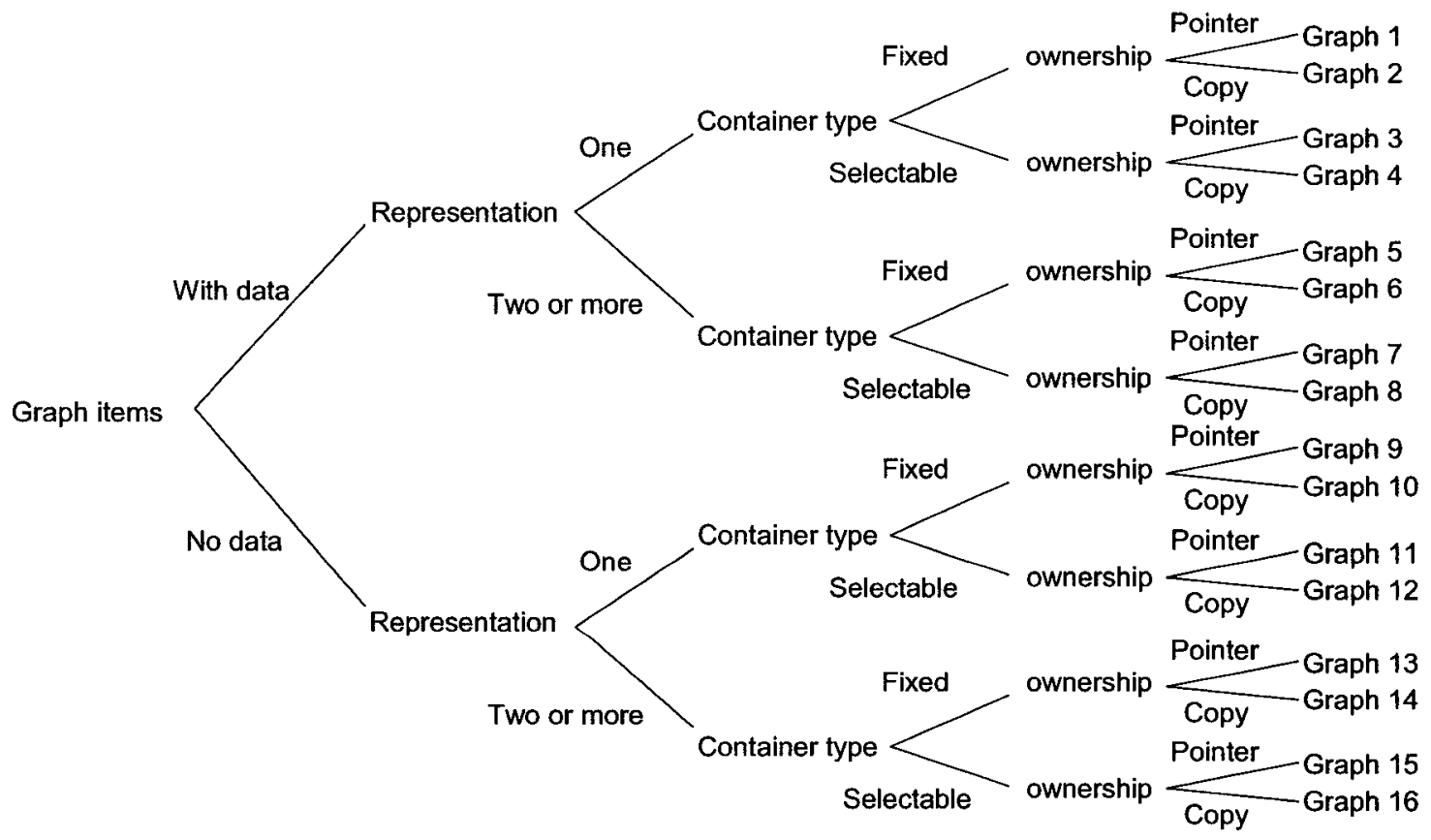

Figure 5-1 Decision tree for graph domain 
Given such a decision tree, tests can be generated for each of the sixteen different types of graphs identified in figure 5-1. It is important to note however this decision tree is still very distant from the intricacies found in the specializations of the integrated feature model. For example, properties are not addressed in this tree, nor complex issues such as parameterized classes! In other words, such a decision tree, though helpful, is still extremely limited.

\subsection{Considering Requirements}

Feature models are semantically limited. In his dissertation, Zhenfeng Wu [41] explores at length the use of contracts to capture feature interactions and feature behavioral dependencies. His work suggests that our integrated feature model for the graph domain should be augmented with such contracts, an arduous task that lies beyond the scope of this dissertation. Here we are interested in leveraging the effort put in the creation of an integrated feature model for the graph domain by proposing how this work can be used to generate tests across the domain at hand.

Based on our extensive investigation of the selected graph libraries, we believe that, in order to truly appreciate distinctions between different libraries addressing a common domain, it is necessary to acquire a deep understanding of the design decisions embedded in those libraries. The integrated feature model is a starting point. Beyond this model, different approaches are possible. For example, in figures 5-2 and 5-3 we use the Goal-oriented Requirement Language (GRL) [14] in order to tie some specific features to goals and "soft goals". The former correspond to functional requirements; the latter, to non-functional ones. Let us briefly elaborate. 


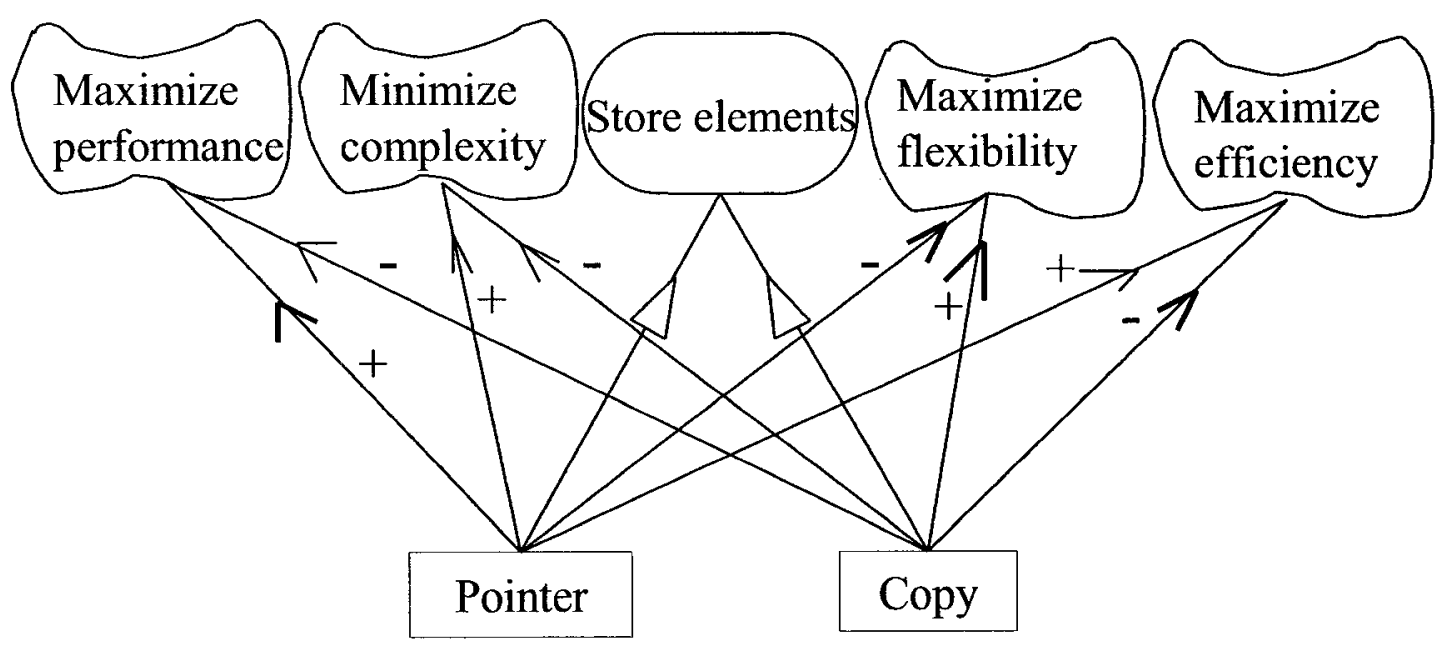

Figure 5-2 GRL model for Ownership

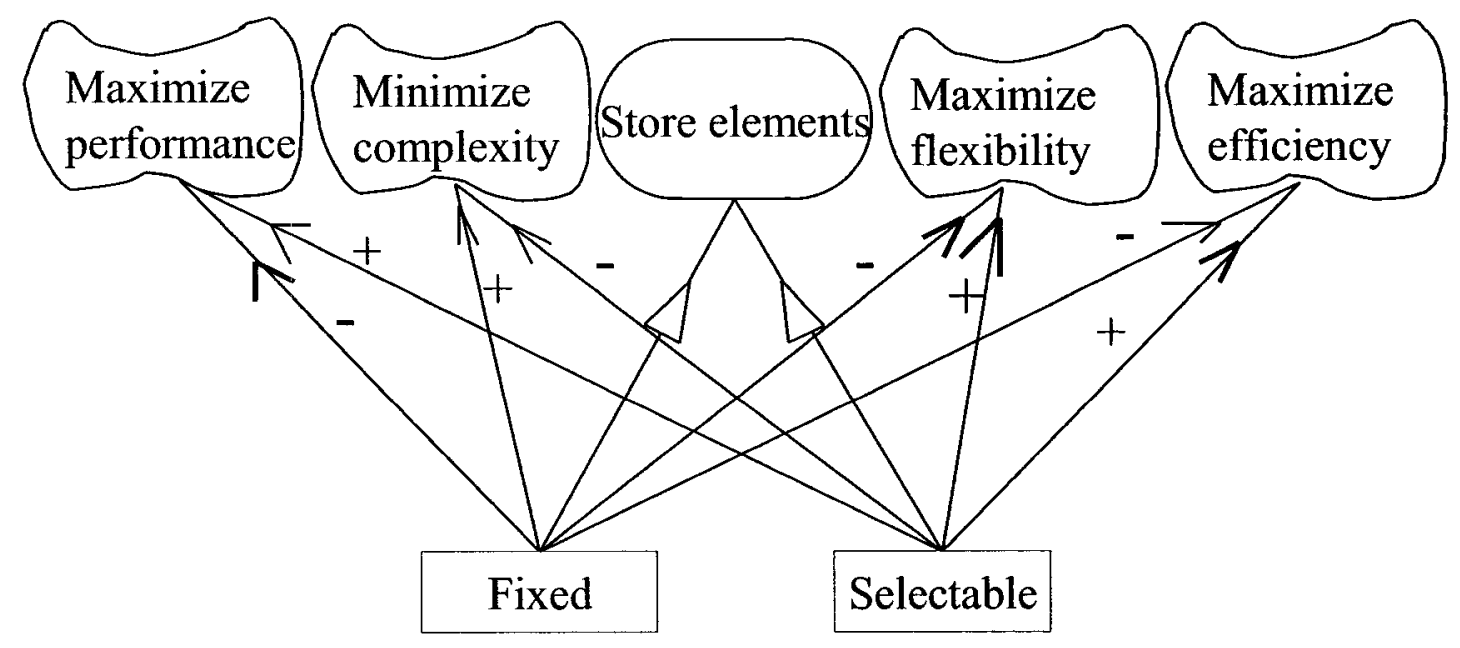

Figure 5-3 GRL model for container type

GRL is a language for supporting goal-oriented modeling and reasoning with requirements, especially for dealing with non-functional requirements. It provides constructs for expressing various types of concepts that appear during the requirement engineering process. There are three main categories of concepts: intentional elements, links, and actors. The intentional elements in GRL are goal, task, soft-goal, and resource. They are intentional because they are used for 
models that allow answering questions such as: why particular behaviors, informational and structural aspects were chosen to be included in the system requirements, what alternatives were considered, what criteria were used to deliberate among alternative options, and what the reasons were for choosing one alternative over the other[9].

We use GRL to tie features to goals and soft goals in order to roughly capture how these features affect the corresponding requirements.

Figure 5-2 considers the feature values of Ownership in our integrated feature diagram. This feature has two values: Pointer and Copy. Both of them can fulfil the functional requirement to store elements (i.e., graph items). Storing elements by pointers can result in better performance and better efficiency than storing elements by copies since there is no need to call copy constructors (in $\mathrm{C}++$ !). Storing elements by pointer can also simplify the complexity of the code, for the same reason. On the other hand, storing elements by pointers will limit flexibility since we need to pay more attention to issues such as dangling pointers.

Figure 5-3 shows the relationships between the feature values of feature container type and requirements. Container type has two values: Fixed and Selectable. Either a fixed container or a selectable container can fulfil the functional requirement to store elements (graph items). We can get better performance by choosing a selectable container type since we can use different containers with different complexity in different situations. In other words, the possibility of selecting a container for the implementation of a graph opens the door to 
optimization of performance through appropriate selection of specialized implementations. For the same reason we can get better flexibility and efficiency by choosing a selectable container. But choosing a fixed container typically results in less complexity in the code and thus better understandability.

Table 5-3 shows how variation points in our feature diagram impact on performance and modifiability.

Table 5-3 impacts of variation points on performance and modifiability

\begin{tabular}{|l|l|c|c|}
\hline \multirow{4}{*}{ Vertex \& edge } & & Performance & Modifiability \\
\hline Representation & With data & - & + \\
\cline { 2 - 4 } & (One) & - & - \\
\cline { 2 - 4 } & $\begin{array}{l}\text { (two or } \\
\text { more) }\end{array}$ & + & - \\
\hline Container type & Fixed & - & + \\
\cline { 2 - 4 } & Selectable & + & + \\
\hline Container ownership & Pointer & + & - \\
\cline { 2 - 4 } & Copy & - & + \\
\hline
\end{tabular}

The point to be grasped is that, beyond obtaining functional tests out of a feature diagram (and its satellite models such as contracts), one must not forget that features can be tied to non-functional requirements (e.g., using GRL) and that, in turn, this can be used to generate some tests. For example, we can envision the development of a suite of tests pertaining to performance (e.g., with respect to traversal) in a graph. This test suite can then be applied to the different categories of graphs identified in table 5-3 in order to verify whether the affect relations of that table are indeed correct or not. (For example, does the limitation of having only one underlying representation indeed hinder performance). Similarly, a test 
suite across the domain could be developed to assess modifiability (though how to do this is not obvious at all!!). And, most importantly, such test suites for different non-functional requirements would directly proceed from the integrated feature diagram put forth for the domain. (Technically, it appears that features play the role of equivalence classes [17]: each feature defines a family of equivalent behaviors.)

\subsection{Scenarios}

Finally, following the work of de Bruin and van Vliet [40], one could hope to obtain functional tests out of a feature model by investigating how features can be assembled into scenarios in order to produce functional tests. We briefly carried such a study, which is summarized in Figures 5-4 through 5-8. Intuitively, these figures try to capture the different decisions required to select a graph. The figures are not meant to be correct or complete, merely to give an idea of how variability could be captured in use case maps [15].We assume the reader has familiarity with the use case map notation[8]. In these figures, variation points are made to correspond to stubs (as has been suggested by several authors).

The conclusion of our investigation of this approach is that it does not lead to tests that would be any different from those obtained in the decision tree of section 5.2 . One has to understand that scenarios are meant to correspond to usages of a system. But the modeling work carried out in this dissertation addresses the structural properties of a graph independently of a context of use! Thus, the scenarios we came up with turned out to be of little value since, we repeat, in our opinion they do not add anything to the knowledge captured in the decision tree of 
section 5.2. Similarly, Wei Shi [39] abandoned the testing of leader election in rings using scenarios because the scenarios she could come up with did not add much to the information already in her feature model.

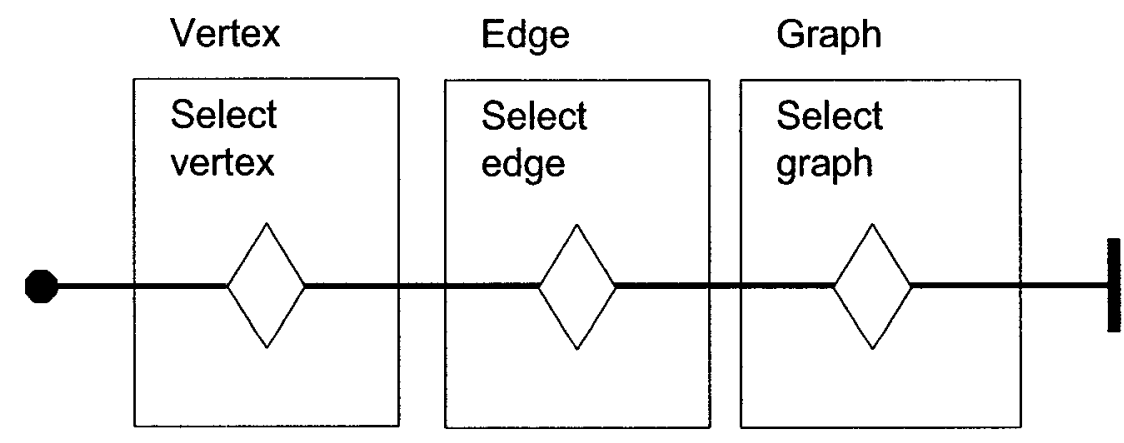

Figure 5-4 UCM model - graph selection

Figure 5-4 states that the selection of a library requires selecting a type of vertex, a type of edge, and a type of graph representation. This is already an oversimplification of the integrated feature model we have presented.

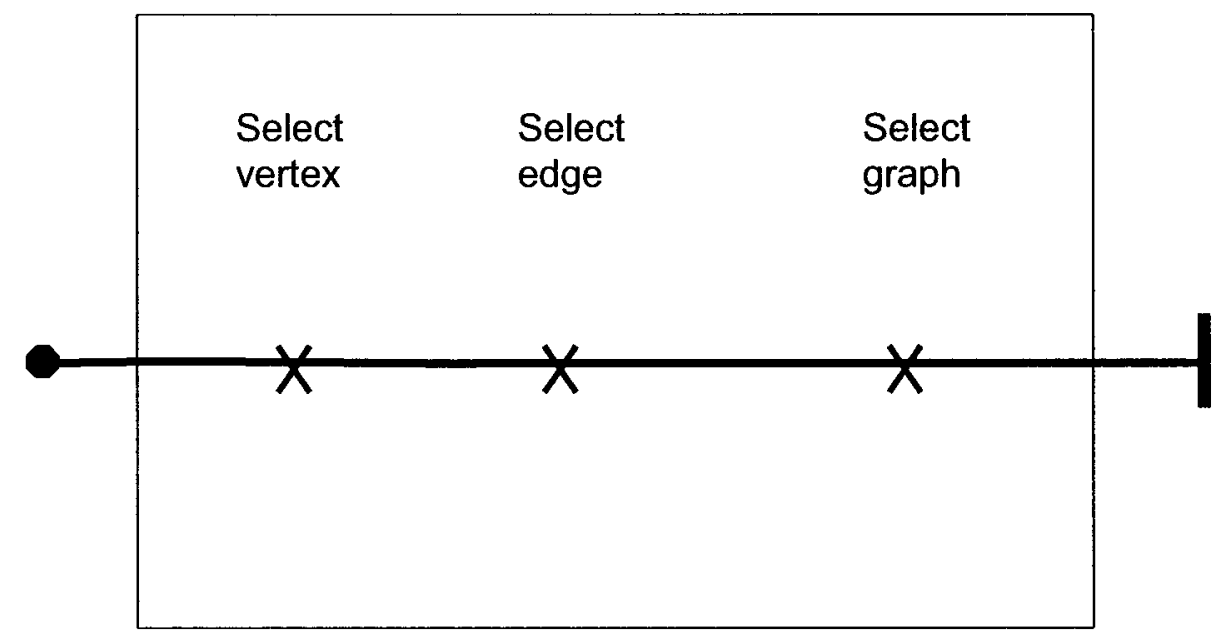

Figure 5-5 UCM model - GTL-G

In GTL-G, everything is fixed; thus library selection boils down to specific choices corresponding to specific functionality (captured by Xs in figure 5-5). 


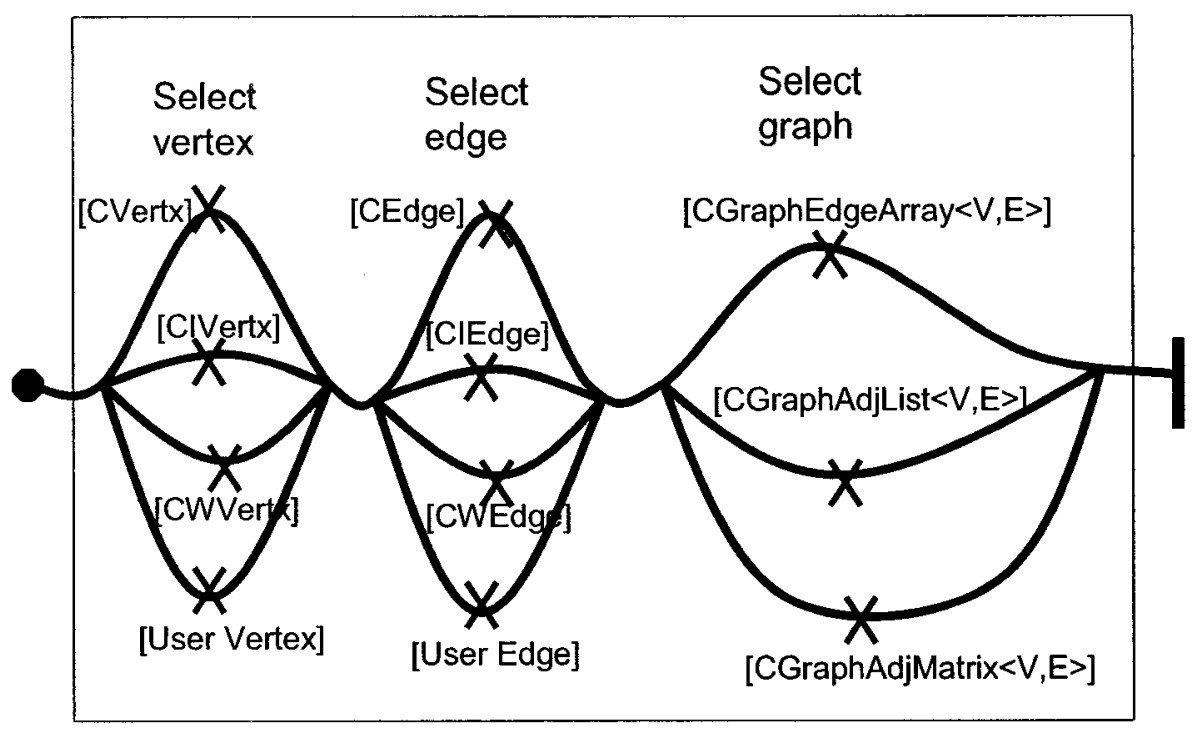

Figure 5-6 UCM model - GTL-R

GTL-R is more complicated than GTL-G. It has four vertex types, four edge types and three graph representations to choose from. This is what figure 5-6 models.

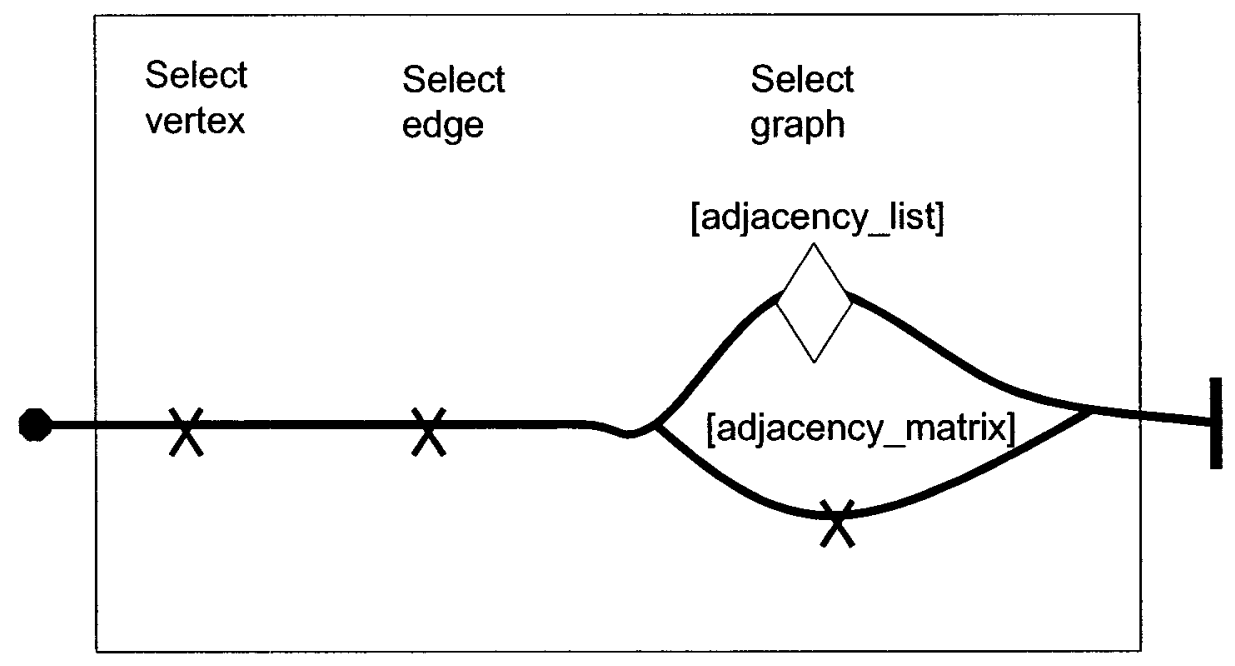

Figure 5-7 UCM model - BGL

BGL has simple vertex and edge type. But has two graph representations to choose. And there are stubs inside graph representations since we need to choose the container type inside graph representation. 


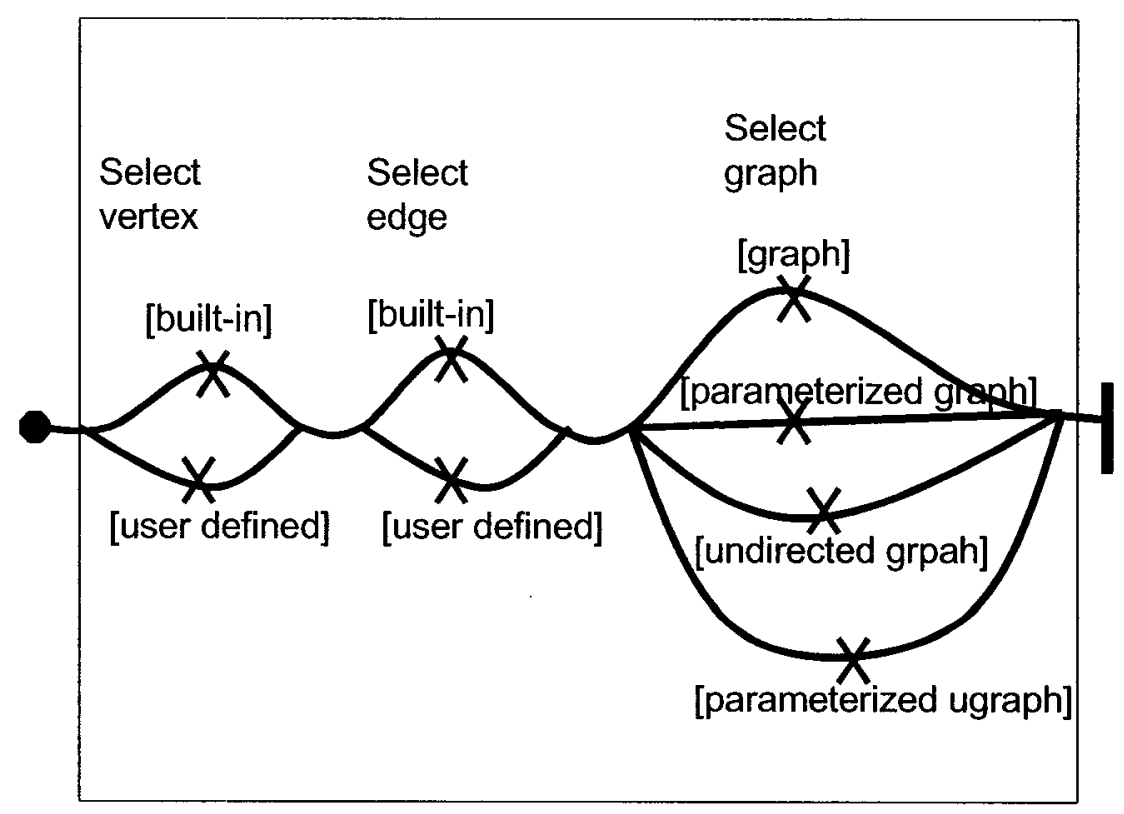

Figure 5-8 UCM model - LEDA

Finally, LEDA has two vertex type, two edge type and four graph types to choose.

The point to grasp, we repeat, is that these use case maps do not offer any new information on top of what the integrated feature model itself (or its corresponding decision tree) capture. In fact, these use case maps incorrectly force the designer to choose a specific order for the decisions (vertex then edge then representation).

This is not a flaw inherent to the notation: we chose to present simplistic scenarios though more complex ones are definitely possible. But even the latter would not address anything more than what is there, namely feature selection. And this where this approach falls short: scenarios, we repeat, are useful when contexts of use are available, which is generally not the case for libraries (viewed as domains). We do want to add, however, that, should scenarios be useful to assemble features into relevant use cases, then using the technique of path sensitization [17], we 
could traverse these scenarios to obtain tests that we believe would be semantically much richer than coming out of a decision tree.

In conclusion, our brief investigation of test generation from feature models suggests that such models must be complemented (with artifacts such as GRL diagrams, contracts and scenarios) in order to facilitate the production of tests. But this is a problem that lies beyond the scope of this dissertation. 


\section{Conclusions}

\subsection{Recapitulation of Contributions}

In this dissertation, we developed feature models in order to facilitate the reuse of a set of independently developed $\mathrm{C}++$ graph libraries. Our purpose is to offer library users an alternative to having to study in detail the code of a library before deciding whether or not and how to reuse its components. Our fundamental postulate is that domain engineering, by directly addressing variability, does facilitate reuse.

First, we built independently a feature model for each of the selected graph libraries. We focused on representational issues. Individually, each of these models provides a conceptual road map to its library. More precisely, instead of class-oriented descriptions, the potential library user will have available a summary of the different representational options that this library offers (and thus of some of the design decisions this user will have to take).

Second, we have built an integrated domain model (and prototyped its corresponding generator). And we have shown how each selected library 'fits into' this unified conceptualization.

Summarizing one possible application of our contributions we remark that faced with a family of similar libraries, developing an integrated feature diagram for this domain and then documenting how each of the libraries fits into this integrated model constitutes a novel approach to the library selection problem. The integrated feature model for our graph domain captures variability across this domain. Then 
individual feature models for each library highlight the design decisions of this library within the conceptual framework associated with the domain. This set of integrated feature models is created once and allows eventual potential users to compare the different libraries using a shared conceptualization. We offer some sample comparison tables we believe do not exist in the literature.

Also, we have hinted, in the previous chapter, to the possibility of augmenting feature diagrams with testable models in order to make more systematic the production of tests from feature models. This leads us to discuss some of the future work we envision.

But first, our conclusion is that, as trivial as it may seem, the creation of feature models requires in-depth study of large amounts of code in order to build corresponding conceptual maps (in the form of feature models). Such maps, which directly capture variability, typically do not exist. And we postulate that they do facilitate the reuse of the components of a library. Furthermore, should an integrated feature model be built across the libraries, potential users of these libraries will readily have a common conceptualization through which they can compare the libraries under study. Again, to the best of our knowledge, this is novel result.

\subsection{Future work}

Building feature models for individual libraries and even more so an integrated feature model in which the libraries of the domain at hand are then "fitted" represents a significant investment of time and requires some expertise. While 
some may immediately appreciate the benefits of a unified conceptual

framework across a domain, several may question whether such an investment is warranted in light of what appears to be pretty trivial diagrams of little use! Czarnecki and Eisenecker [11] explain elsewhere the importance of domain engineering in the context of software reuse. Our research groups promotes another aspect, namely quality engineering [16]. It is from this perspective that we briefly discuss possible future work.

First, when comparing our work to that of Juemin Yu [31] and Wei Shi [39], we realize that domains can vary considerably in nature. Graph and sequential containers libraries form domains of reusable components that are dissociated from their context of usage (that is, the algorithms and applications that use these components). Feature models for such domains tend to be 'structural': variability focuses on how the reusable components can be formed more than on their behavior proper. Conversely, algorithmic domains, such as leader election in rings, appear to lead feature models where variability is essentially behavioral. Though each library to study represents an enormous amount of time to 'digest', we would like to consider other families of libraries in order to confirm whether this distinction between structure-oriented and behavior-oriented domains is confirmed or not. Should this be the case, then we envision the investigation of 'organizational' patterns for these two types of domains. In other words, the creation of integrated feature models across a family of libraries could possibly be guided by a set of typical patterns for such models. For structure-oriented families, our work suggests the following 'meta' features: i) elements (i.e., the entities that form the structure, such as vertices 
and edges for graphs), ii) representation (i.e., the underlying data structures of choice), and iii) properties (as discussed in chapters 4 ). In turn, we hope that adopting some conventions for the creation of integrated feature models could lead to easier production of tests, which we discuss next. (The creation of integrated feature models is an exercise in conceptual integration and our future investigations could also look at work in Artificial Intelligence. In a distant future, it would be extremely useful to have intelligent mining tools that extract unified conceptualizations from a set of libraries!)

Once a set of integrated feature models is obtained for a domain (and its individual members), the initial investment of time can be leveraged by using this set of models to produce tests across the domain (which is probably much more efficient than producing tests per libraries). Extending reuse to reuse of tests across a domain is the mandate of our research group. But, as previously mentioned, feature models per se do not necessarily lead to tests: these models need to be complemented. In the short term, we believe it would be most urgent to develop feature contracts [41]. Such contracts would definitely improve the information available to a potential library user in that they would allow this user to quickly assess the level of coupling in different libraries. A thorough investigation of how to relate feature models to functional and non-functional requirements (as hinted at in [16]) is also a short-term goal: no quality engineering is possible without some traceability back to requirements. We have merely scratched the tip of the proverbial iceberg in the previous chapter. More work is required with respect to traceability links and with respect to the generation of tests per se. Ultimately, we envision an application engineering 
environment in which feature models and their satellite models could be input and that would somehow use these models to generate tests for the domain. This is one of the most serious omissions we find with current libraries (and a multitude of other software systems): they never include a test suite. We do believe that having such test suites would improve understandability of individual libraries, and that, should the test suites hold across the domain, test case generation could be more productive.

Finally, we would like to investigate further the development and testing of a generator for structure-oriented domains such as graph and container libraries. The creation of such a generator could possibly greatly simplify testability and portability issues. For example, we believe that the $\mathrm{C}++$ generator we have developed will eventually be useful in simplifying the production of actual test cases. And should we develop the generator in a different language (with the proviso of 'porting' what makes sense in the target language, e.g., does this language support pointers?), it is possible we would then have the possibility of reusing the source functionality without having to depend on the presence of "equivalent" libraries in the target language. For example, having a Java generator for graph libraries would alleviate the problem of searching for such libraries in Java! 


\section{References}

[1] Kenneth H. Rosen. Discrete Mathematics and Its Applications. Fourth Edition. American Telephone and Telegraph Company, AT\&T Laboratories Division.

[2] Thomas H. Cormen, Charles E. Leiserson and Ronald L. Rivest. Introduction to Algorithms. The MIT Press. Cambridge, Massachusetts London, England.

[3] http://infosun.fmi.uni-passau.de/GTL/

[4] Kurt Mehlhorn, Stefan Näher. A Platform for Combinatorial and Geometric Computing

[5] http://www.gtl.kis.ru/

[6] http://www.boost.org/libs/graph/doc/table_of_contents.html/

[7] http://www.algorithmic-solutions.com/enleda.htm/

[8] http://www.usecasemaps.org/urn/

[9] http://www.cs.toronto.edu/km/GRL/

[10] http://www.usecasemaps.org/

[11]K. Czarnecki and E. Eisenecker. Generative Programming. Addison-Wesley, 2001

[12] Y. Smaragdakis and D. Batory, Implementing Layered Designs with Mixin Layers, ECOOP '98.

[13]E. Gamma, R. Helm, R. Johnson, and J. Vlissides, Design Patterns: Elements of Reusable Object-Oriented Software. Reading, MA: Addison-Wesley, 1995.

[14]Lin Liu, and Eric Yu. From Requirements to Architectural Design-Using Goals and Scenarios, ICSE-2001 Workshop: From Software Requirements to Architectures (STRAW 2001) May 2001, Toronto, Canada. pp. 22-30 
[15]D. Amyot and G. Mussbacher, "Bridging the Gap Between Requirements and Design with Use Case Maps,", www.usecasemaps.org, Ottawa, Canada, 2001.

[16] J-P. Corriveau and T. McClean, "Addressing Quality in System-Family Engineering", submitted to GPCE_03.

[17]R. Binder. Testing Object-Oriented Systems. Addison-Wesley, 2000.

[18] http://www.cs.sunysb.edu/ algorith/implement/LEDA/implement.shtml

[19] http://www.cs.sunysb.edu/ algorith/implement/graphbase/implement.shtml

[20]C. Atkinson et al., Component-Based Product-Line Engineering with $U M L$ :Addison-Wesley, 2001.

[21]J. Bosch, Design \& Use of Software Architectures: Adopting and Evolving a Product Line Approach: Addison-Wesley, 2000.

[22]P. Clements and L. Northrop, Software Product Lines: Practices and Patterns:Addison-Wesley, 2001.

[23]D. Fey, R. Fajta, and A. Boros, "Feature Modeling: A Meta-Model to Enhance Usability and Usefulness, " presented at Software Product Lines, Second International Conference, San Diego, CA, 2002.

[24]J-P. Corriveau, "Envisioning Domain-Driven Validation", TOOLS-USA 03.

[25] J. Bayer, O. Flege, P. Knauber, R. Laqua, D. Muthig, K. Schmid, T. Widen, and J.-M. Debaud, "PuLSE: A Methodology to Develop Software Product Lines," presented at Symposium on Software Reuse (SSR'99), 1999.

[26] J. Bosch, Design \& Use of Software Architectures: Adopting and Evolving a Product-line Approach: Addison-Wesley, 2000.

[27]D. Parnas, "On the Design and Development of Program Families," IEEE Transactions on Software Engineering, vol. SE-2, no. 1, pp. 1--9, 1976. 
[28] D. M. Weiss and C. T. R. Lai, Software Product-line Engineering: A Family-Based Software Development Process. Reading, MA: Addison-Wesley, 1999.

[29]L. Geyer, "Feature Modeling Using Design Spaces," presented at 1st German Workshop on Product Line Software Engineering, Kaiserslautern, Germany, 2000.

[30]K. C. Kang, S. G. Cohen, J. A. Hess, W. E. Novak, and A. S. Peterson, "Feature-Oriented Domain Analysis (FODA) Feasibility Study," Software Engineering Institute, Carnegie Mellon University, Pittsburgh, PA CMU/SEI-90-TR-21, 1990.

[31]Juemin Yu, Domain Engineering for Sequential Containers, Master's thesis, School of Computer Science, Carleton University, January 2004.

[32] http://www.c3.lanl.gov/ reid/Web papers/genGraphToolTR/node12.html

[33] http://www.cs.brown.edu/people/jak/proglang/cpp/stltut/

[34] http://msdn.microsoft.com/library/

[35] http://www.cis.temple.edu/ wolfgang/MFC-STLPaper.PDF

[36]Ed Yourdon, Death March, Prentice Hall, 1999.

[37] J.-P. Corriveau, Time-Constrained Memory, LEA, 1995

[38] Rational ROSE Real-time, www.rational.com

[39] W. Shi, System Family Engineering for Leader Election in Rings, Master's thesis, School of Computer Science, Carleton University, December 2003.

[40]H. deBruin and H. van Vliet, Quality-Driven Software Architecture Composition, ECBS 2002.

[41]Z. Wu, Capturing Coupling Information in Domain Engineering, Master's thesis, School of Computer Science, Carleton University, December 2003. 
[42]I. Jacobson, Object-Oriented Software Engineering, Addison-Wesley, 1992.

[43]H. van Vliet, Software engineering: principles and practice. Wiley, 2nd edition, 2002

[44] Object Management Group, MDA Guide version 1.0, OMG whitepaper, http://www.omg.org/docs/omg/03-05-01.pdf

[45]E. Evans, Domain-Driven Design: Tackling Complexity in the Heart of Software, Addison-Wesley, 2003.

[46] N. Josuttis, The C++ Standard Library: A tutorial and Reference, Addison-Wesley, 1999

[47]R. Wirfs-Brock, A. McKean. Object Design: Roles, Responsibilities, and Collaborations. Addison-Wesley, 2003.

[48]H. Gomaa, Designing Concurrent Distributed, and Real-Time Application with UML. Addison-Wesley, 2000.

[49]K. Kang, S. Cohen, J. Hess, W. Nowak and S. Peterson, Feature-Oriented Domain Analysis (FODA) Technical Report, CMU/SEI-90-TR-21, Software Engineering Institute, Carnegie Mellon, Pittsburgh, PA, November 1990. 